
. 431.0588

AAPG

1912THE PUBLIC LIBRARY OF THE

\section{DISTRICT OF COLUMBIA}

\section{Reference List No. 11}

\section{CONTEMPORARY AMERICAN ARTISTS}

References to literature in books and periodicals on the exhibitors at the fourth biennial exhibition of contemporary American art, Corcoran Gallery of Art, I7 December IgI2, to 26 January I9I3.

The material listed may, for the most part, be found at

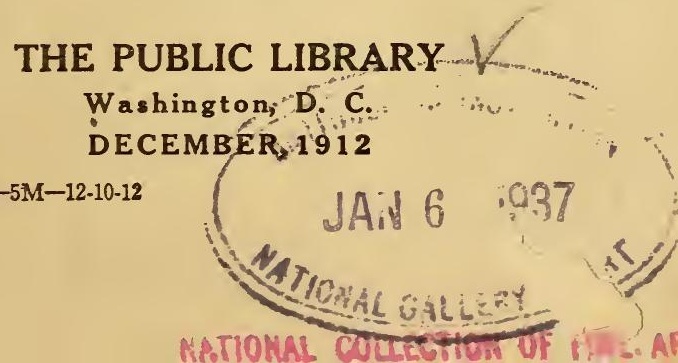

P. L. 202-5M-12-10-12 


\section{Contemporary American Artists}

The following books have material on some of the artists represented. In cases where the criticism is somewhat extended a specific reference to that fact will be made under the individual treated.

For passing references readers may consult the indexes to such books as those by Caffin, Hartmann, Isham, etc. Nearly all the artists exhibiting are represented in the new "Who's Who in Art."

\section{Books}

Benjamin, S. G. W. Art in America. I880. W83-B436.

Brinton, Christian. Modern artists. I908. Wio-9B776.

Caffin, C. H. American masters of painting. I902. WIO-gCiI2.

Caffin, C. H. Story of American painting. 1907. WP-Cirze.

Child, Theodore. Art and criticism. I892. W-C435.

Clement, Mrs. C. E. Women in the fine arts. I904. Wio-9C 59 w. 
GACox, Kenyon. Old masters and new. 1905. W I0-9C8360.

Dewhirst, Wynford. Impressionist painting. 1904. WP-D5I4i.

D'Anvers, N. pseud. Representative painters of the I9th century. 1899. Wro9D238.

Downes, W. H. Twelve great artists. 1900. WIO-९D758.

Hartmann, Sadakichi. History of American Art. 2 v. 1902. W83-H255.

Henderson, H. W. Art treasures of Washington. I9I2. WC859- $\mathrm{H}_{3} 83$.

Isham, Samuel. History of American painting. I907. WP-4M984.

McSpadden, J. W. Famous painters of America. 1907. Wio-9M246.

Muther, Richard. History of modern painting. 1907. (v. 4 America.) WP$4 \mathrm{Mg} 84$.

Rummell, John and Berlin, E. M. Aims and ideals of representative American painters. I90I. W83-R866.

Sargent, J. S. Work of Sargent, with intro. by Mrs. Meynell. I903. WP-Sa743. 
Vedder, Elihu. Digressions of V. I9I0. WIO-V5I3.

Who's who in art. I9I2. WI0-5W627. Reference.

\section{Art and Progress}

The following articles in "Art and Progress" relate chiefly to recent exhibitions in which mary of the younger artists are discussed, who are not noted elsewhere:

America at Rome. Morris. Oct., 'I I American marine painters. Nov., 'Io. Art in the West. Curley. May, 'II. Art outlook. Alexander. May, 'I2.

Carnegie Institute's exhibitions. June, 'II and 'I2.

Chicago Art Institute exhibitions. Dec., 'Io.

National Academy. May, 'ıo and Feb., 'I2.

National Gallery of Art. April, 'ıo.

Pennsylvaria Academy exhibitions. Jan., 'Io and April, 'I2. 


\section{JOHN W. ALEXANDER}

Books: Caffin-Story of Amer. Painting, Hartmann, and Isham.

Magazines: Craftsman Io:46 (April, 'o6) ; Critic 35:609 (July, '99) ; Fine Arts Jour. 25 :I3 (Jan., 'II) ; Harper's 99:694 (Oct., '99) ; House Beautiful I $5: 67$ (Jan., '04) ; Int'l Studio 34 :sup. 85 (May, 'o8); 42 : I79 (Jan., 'I I) ; Munsey 39:744 (Sept., 'o8); Outlook 95 :I70 (28 May, 'ıo) ; Scribner's 25:340 (Mar., '99); Studio 20:7 I (July, 'oo) ; World's Work 9:5632 (Jan., 'o5).

\section{CLIFFORD W. ASHLEY}

Harper's I 2 :670,832 (Apr. \& May, 'o6).

\section{HUGO BALLIN}

Art and Progress I:I45 (April, 'IO); 3:699 (Sept., 'I2) ; Critic $47: 496$ (Dec., '05); Int'l Studio 24 :sup. 22 (Nov., '04).

\section{GIFFORD BEAL}

Brush \& Pencil, Feb., 'o7; Int'1 Studio 44 :sup. 29 (Aug., 'II).

\section{JOHN W. BEATTY}

Canadian Mag. 26:346 (April, 'o6). 


\section{CECILIA BEAUX}

Books : Clement, Hartmann, Isham, and Rummell.

Magazines : Century 80:58I (Aug., 'Io); Harper's Bazar 45 :II9 (Mar., 'II); Int'1 Studio 4I sup. 3 (July, 'Io) ; House Beautiful II :I75 (Feb., '02); Critic 47:39 (July, '05) ; Scribner's $22: 477$ (Oct., '97) ; Studio I7:215 (Sept., '99).

\section{GEORGE BELLOWS}

Art and Progress 3:679 (Aug., '12); Current Lit. I53:342 (Sept., 'I2).

\section{FRANK W. BENSON}

Books: Isham-Amer. Painting.

Magazines: Art and Decoration, Mar., 'II; Brush and Pencil 6:I45 (July, 'oo); Harper II9:I05 (June, 'o9); Int'l Studio $35: 99$ (Oct., 'o8); New Eng. Mag., 38: 328 (May 'o8) ; St. Nich. 36:883 (Aug., 'o9).

\section{LOUIS BETTS}

Sketch Bock 6:I7 I (Dec., 'o6). 


\section{HUGH H. BRECKENRIDGE}

Academy Notes 3:I3I,I50 (Jan., 'o8); Book News Aug., '06; Int'l Studio 37: sup. 34 (Mar., 'o9).

\section{GEORGE E. BROWNE}

Brush and Pencil May, '04; Int'1 Studio 36:286 (Feb., 'o9).

\section{GEORGE DeF. BRUSH.}

Books: Caffin, Hartmann, and Isham.

Magazines: Century 21 :600,638 (Feb., '92) ; Critic $47: 134$ (Aug., '05); Int'l Studio 34 :sup. 47 (April, 'o8).

\section{MARY CASSATT}

Books: Isham.

Magazines: Craftsman 19:540 (Mar., 'II); Good Housekeeping 50:I4I (Feb., 'Io) ; Harper's Bazar $45: 490$ (Nov., 'I I); Harper's I23:596 (Sept., 'I I) ; Int'1 Studio 27 :sup. I (Nov., '05); Current Lit. 46:167 (Feb., 'o9) ; Scribner's I9:353 (Mar., '96); World Today 21 :I659 (Jan., 'I2).

\section{WILLIAM M. CHASE.}

Books: Benjamin, Hartmann, Isham, McSpadden, and Rummell. 
Magazines: Craftsman 18:33 (April, 'Io); House Beautiful 25:50 (Feb., 'o9); Critic 48:5I5 (June, 'o6); Harper's II 3: 698 (Oct., 'o6); Rev. of Revs. 36:693 (Dec., '07) ; Int'1 Studio 39 :sup. 29 (Dec., 'og); Studio 2 I :I5I (Dec. 'Oo).

\section{CHARLES S. CHAPMAN}

Scribner's $42: 305$ (Sept., 'O7).

\section{RALPH CLARKSON}

Brush and Pencil I :95 (I897).

\section{CHARLES C. COLEMAN}

Archit. Record 22:455 (Dec., 'O7) ; 2 I : 36I (May, 'o7) ; I8:300 (Oct., 'O5); 24: 449 (Dec., '08).

\section{COLIN C. COOPER.}

Art and Progress 3:60I (June, 'I2); Booklovers 5:327 (March, '05); Fine Arts Jour. Oct., 'o3.

\section{PAUL CORNOYER}

Current Lit. 47:54 (July, 'o9).

\section{E. JRVING COUSE}

Academy Notes 2:22 (July, 'o6); Craftsman I8:619 (Sept., 'Io); Palette and Bench 2:45 (Dec., 'og). 


\section{BRUCE CRANE}

Books : Rummell.

Magazines: Brush and Pencil II:I (Oct., '02).

\section{CHARLES H. DAVIS}

Books: Child, Hartmann, and Rummell.

Magazines: Brush and Pencil. May. 'O3: Century 48:319 (June, '94); New Eng. Mag. $33: 423$ (Dec., 'o2).

\section{JOSEPH DeCAMP}

Arts and Decoration. April. 'it : Century 78 :64I (Aug.. 'on): Art and Progress 3:539 (April. 'I2); New Eng. Mag. 39: 238 (Oct., 'o8).

\section{EDWARD DUFNER}

Academy Notes 2:I2 (June, 'o6).

\section{LOUIS P. DESSAR}

Brush and Pencil 5:97 (Dec., '99) : Harper I $22: 282$ (Jan., 'I I).

\section{CHARLES M. DEWEY}

Books: Rummell.

Magazines: Harper I I4:444 (Feb., '07). 


\section{THOMAS W. DEWING}

Books: Hartmann, and Isham.

Magazines: Art and Progress 3:729 (Oct., 'I2); Critic 48:418 (May, 'o6); Harper's 9I:I66 (July, '95); I I6:7I4 (April, 'o8).

\section{PAUL DOUGHERTY}

Academy Notes 7:20 (April, 'I2); Int'1 Studio 30 :I80 (Dec., 'o6); 36 :sup. 3 (Nov., 'o8).

\section{FRANK V. DuMOND}

Art and Progress 4:772 (Nov., 'I2).

\section{THOMAS EAKINS}

Books : Caffin, Hartmann, and Isham.

\section{LYDIA F. EMMET}

Art and Progress 2:I25 (Mar., 'II); Arts and Decoration 2:4I5 (Oct., 'Iz); Delineator 77 :89 (Feb., 'I I ).

\section{BEN FOSTER}

Artist 29 :sup. 20 (Jan., 'OI).

\section{FREDERIC C. FRIESEKE}

Art and Progress 3:747 (Oct., 'I2); Harper I 18:29I (Jan., 'og); Int'1 Studio $43: 263$ (June, 'II). 


\section{DANIEL GARBER}

Maitres Contemporains 59:Io (I912).

\section{WALTER GAY}

Books : Child.

Magazines : Century $53: 316$ (Dec., '96) ; Int'l Studio 35 :I50 (Aug., 'o8) ; Mag. of Art 18:227 (April, '95).

\section{ALBERT L. GROLL}

Craftsman 9:826 (Mar., 'o6) ; Int'1 Studio 28 :sup. 78 (May, 'o6) ; 29:270 (Sept., 'о6).

\section{PHILIP L. HALE}

Arts and Decoration 2:I8I (Mar., 'I2); New Eng. Mag. 44:196 (April, '08); World Today I4:59 (Jan., 'o8).

\section{JOHN M. HAMILTON}

Scribner's 27:733 (June, 'oo).

\section{ALEXANDER HARRISON}

Books: Child, Dewhurst, Hartmann, Isham, Muther, and Rummell.

Magazines: Brush and Pencil 4:I33 (June, '99); Critic 43:554 (Dec., 'o3); Studio I3:248 (May, '98). 


\section{BIRGE HARRISON}

Academy Notes 4:II3 (Jan., 'o9); Art and Progress $3: 379$ (Nov., 'I I) ; Craftsman $13: 397$ (Jan., 'o8) ; Int'l Studio 44:3 (July, 'I I) ; Scribner's $42: 576$ (Nov., 'o7).

\section{CHILDE HASSAM.}

Books: Hartmann.

Magazines: Art and Progress 2:137 (Mar., 'II); Brush and Pencil 8:I4I ( May, 'or) ; House Beautiful 23:19 (Jan., 'o8) ; Int'l Studio 29:267 (Sept., 'o6); 45: sup, 29 (Dec., 'I I).

\section{CHARLES W. HAWTHORNE}

Art Interchange 52:138 (June, '04); Brush and Pencil 4:255 (Aug., '99) ; 15: 227 (April, '05); Fine Arts Jour. 27:198 (Mar., 'I2) ; Int'l Studio 26:26I (Sept., '05) ; 37 :sup. 65 (May, 'o9).

\section{ROBERT HENRI}

Books: Hartmann.

Magazines: Arts and Decoration $2: 213$ (April, 'I2): Craftsman 21 :454 (Jan., 'I2); Critic 49:I30 (Aug., 'o6) : Current Lit. 52: 464 (April, 'I2); Harper's I 7 :430 (Aug., 
'08); I25:706 (Oct., 'I2); Independ. 64: I 427 (June 25, 'O8); Int'l Studio 30:182 (Dec., '06).

\section{HENRY S. HUBBELL}

Century 70:635 (Aug., '05); Harper's I I :289, (Jan., 'o9); World Today II :I I 39 (Nov., '06).

\section{EDWARD T. HURLEY}

Int'l Studio 29 :sup. 63 (Sept., '06).

\section{CECIL JAY}

Fine Arts Journal $25: 72$ (Feb., 'II).

\section{JOHN C. JOHANSEN}

Int'l Studio $26: 264$ (Sept., 05) ; 42 :sup. 3 (Nov., 'IO); Sketchbook 5:53 (Oct., '05).

\section{W. SARGENT KENDALL.}

Arts and Decoration I :I5 (Nov., 'IO); Century $50: 478$ (July, '95); Harper's II7: 568 (Sept., 'o8);

\section{ERNEST IAWSON}

Current Lit. $42: 406$ (April, 'o7). 


\section{JONAS LIE}

Century 8I :390 (Jan., 'II) ; Craftsman I3:I35 (Nov., 'O7) ; 21 :455 (Jan., 'I2); Current Lit. 52 :222 (Feb., 'I2).

\section{ROBERT MacCAMERON}

Harper's I 18:286 (Jan., 'o9). WALTER MacEWEN

Maitres Contemporains 46:8 (I908). M. JEAN McLANE

Art and Progress 3:612 (June, 'I2); Harper's II8:292 (Jan., 'og).

\section{MARY MACOMBER}

Art and Progress 2:204 (May, 'II); Int'1 Studio 47 :sup. 60 (Oct., 'I2).

\section{LEWIS H. MEAKIN}

Int'1 Studio 33 :sup. 3 (Nov., 'o7).

\section{GARI MELCHERS}

Books: Brinton, Child, Caffin, and Rummell.

Magazines: Craftsman 20:III (April, 'II) ; Hampton 26:652 (May, 'Ir); Harper's I I4:430 (Feb., 'o7)' Int'1 Studio 3I : 
sup. II (Mar., 'o7) ; 48:27 (Dec., '12); Mag. of Art 24 :I45 (Feb., 'Oo); World's Work I5:I0092 (April, 'o8).

\section{WILLARD L. METCALF}

Booklover's 6:499 (Oct., 05) ; Century 77 :155 (Nov., '08); New Eng. Mag. 39: 374 (Nov., '08); Quarterly Illustrator 3 :93 (Jan., '95).

\section{RICHARD E. MILLER}

Art Review 23 :I5 (Mar., 'I2) ; Int'1 Studio $43: 263$ (June, 'I I).

\section{F. LUIS MORA}

Craftsman I7:402 (Jan., 'Io) ; Collector and Art Critic $4: 201$ (May, 'o6); Harper's I23:888 (Nov., 'II).

\section{HERMANN D. MURPHY}

Brush and Pencil 5:49 (Nov., '99); Sketchbook 6:303 (Nov., 'o7).

\section{J. FRANCIS MURPHY}

Books : Rummell.

Magazines Brush and Pencil I0:205 (July, '02). 


\section{ELIZABETH NOURSE}

Art and Progress 2:262 (July, 'II); $3: 746$ (Oct., 'I2) ; Current Lit. $48: 90$ (Jan.,

'Io) ; Century 59:48I (Jan., 'oo) ; Int'1 Studio $27: 24 ;$ (Jan., '06).

\section{GEORGE OBERTCUFFER}

Int'l Studio $43: 275$ (June, 'II).

\section{LEONARD OCHTMAN}

Brush and Pencil 9:65 (IgOI); Fine Arts Jour. I4:315 (Aug., 'O3).

WILLIAM MacG. PAXTON

Int'1 Studio 39 :sup. 46 (Dec., 'og) ; New Eng. Mag. 39:36 (Sept., 'o8) ; World Today 9:I2IO (Nov., '05).

\section{GITTARDO PIAZZONI}

Overland $54: 460$ (Nov., 'o9).

\section{HENRY R. POORE}

Int'1 Studio 37 :sup. 20 (Mar., 'og); New Eng. Mag. 39:604 (Jan., 'o9).

\section{EDWARD W. REDFIELD}

Arena 36:20 (July, 'o6) ; Country Life in America I3:I94 (Dec., 'o7) ; Int'1 Studio 4I :sup. 29 (Aug., 'Io). 


\section{ROBERT REID}

Books : Hartmann, and Isham.

Magazines: Art and Progress 2:157 (April, 'II); Artist 28:45 (June, 'OO); Arts and Decoration 2:I3 (Nov., 'II); Booklovers 6:738 (Dec., 'o5); Century $77: 642$ (March, 'o9); Craftsman 7:699 (Mar., '05) ; Int'1 Studio 36 :sup. I 13 (Feb., 'og).

HENRY REUTERDAHL.

Collier's $45: 22$ (Aug., I3, '10) ; $49: 29$ (May 4, 'I2) ; 47 :19 (April 15, 'II) ; Outlook 96:407 (Oct. 22, 'Io).

\section{OLIVE RUSH}

Woman's Home Comp. 39:20 (April, 'I2).

\section{CHAUNCEY F. RYDER}

Art Review 22:I (Sept., 'II).

\section{JOHN S. SARGENT}

Books : Brinton, D'Anvers, Caffin, Cox, Downes, Hartmann, Isham, McSpadden, Muther, Rummell, and Sargent.

Magazines: Art and Progress 3:742 (Oct., '12) ; Century $52: 163$ (June, '96); Chautauquan 50:8I (Mar., '08) ; Critic 47: 
326 (Oct., '05) ; Current Lit. 39:504 (Nov., '05); Int'l Studio 37 :sup. 55 (April, 'o9); Rev. of Revs. 36:692 (Dec., 'o7) ; St. Nich. 32:1094 (Oct., 'O5); Scribner's 34:5 I5 (Nov., 'o3) ; $52: 509$ (Oct., 'I2); World's Work 7 :4099 (Nov., 'o3).

\section{WILLIAM SARTAIN}

New Eng. Mag. 28:5I (Mar., 'o3).

\section{W. ELMER SCHOFIELD}

Arts and Decoration I :473 (Oct., 'II).

\section{EUGENE E. SPEICHER}

Int'l Studio 46 :sup. I9 (Mar., 'I2).

\section{GARDNER SYMONS}

America : 2:526 (Feb., 26, 'Io).

\section{HENRY O. TANNER}

Brush and Pencil 6:97 (June, 'oo) ; Cosmopolitan 29:I8 (May, 'oO); Current Lit. 45:404 (Oct.. 'o8) ; Independent 65:1600 (Dec., 3I, 'o8); Outlook 64:793 (April 7,


July, 'o9).

\section{EDMUND C. TARBELL}

Arts and Decoration 2:I29 (Feb., 'I2); Art and Progress $2: 20$ (Nov., '10); $2: 93$ 
(Feb., 'II) ; Booklover's 3:749 (June, '04) ; Brush and Pencil 3:I93 (Jan., '99); Critic 48 :I36 (Feb., '06) ; Harper I I2 :702 (April, 'o6); I I7:65 (June, '08); Int'l Studio 32: sup. 75 (Sept., 'o7); New Eng. Mag. 38: 96 (Mar., '08); World Today Ir :I077 (Oct., 'o6).

\section{ABBOTT H. THAYER}

Books: Child, D'Anvers, Hartmann, Isham.

Magazines: Cosmopolitan 32:594 (April, 'o2); Critic 46:422 (May, '05); House Beautiful I3:3 (Dec., 'O2); Int'1 Studio 33 :sup. 8I (Jan., 'o8).

\section{DWIGHT W. TRYON}

Books: Isham, and Rummell.

Magazines: Harper's 9I :I70 (July, '95).

\section{ELIHU VEDDER}

Books: Hartmann, Isham, McSpadden, Rummell, and Vedder.

Magazines: Atlantic 106:403 (Sept., 'Io); Bookman $35: 145$ (April, 'I2); Int'1 Studio 35 :sup. 94 (Sept., 'o8) ; Outlook: 96:693 (Nov. 26, 'Io).

\section{DOUGLASS VOLK}

Century 68:654 (Aug., 'o4). 


\section{ROBERT W. VONNOH}

Books : Hartmann.

Magazines: Arts and Decoration 2:38I (Sept., 'I2) ; Harper's I 16:254 (Jan., '08).

\section{LIONEL WALDEN}

Cosmopolitan 29:20 (May, 'oo).

\section{HORATIO WALKER}

Canadian Mag. I8:496 (April, 'O2); Craftsman 14:138 (May, '08); Harper's I I7 :947 (Nov., 'o8).

MARTHA WALTER

Arts and Decoration I :303 (May, 'I I).

\section{EVERETT WARNER}

Scribner's 46:280 (Sept., 'o9).

\section{FREDERIC J. WAUGH}

Art and Progress 3:588 (May, 'I2); Arts and Decoration I III I (Jan., 'II); Maitres Contemporains pl. 47 (Sept., 'I2).

\section{J. ALDEN WEIR}

Books: Hartmann, and Isham.

Magazines: Arts and Decoration 2:55 (Dec., 'I I) ; Century 57 :956 (April, '99); Cosmopolitan 32:596 (April, '02) ; Harper's I I4:286 (Jan., 'o7). 


\section{WILLIAM WENDT}

Brush and Pencil 6:257 (Sept., 'oo).

\section{IRVING R. WILES}

Century 54:799 (Sept., '97); 82:521 (Aug., 'II) ; Craftsman 18:347 (June, 'ro); Harper's I09:802 (Oct., 'o4); II4: 608 (Mar., '07) ; Rev. of Revs. 4:40 (July, '06).

\section{CHARLES H. WOODBURY}

Academy Notes 7:3I (April, 'I2); Art and Progress 4:76I (Nov., 'I2); Brush and Pencil 6:I (April, 'oo); Int'1 Studio 42 :sup. 7I (Feb., 'II).

Nearly all of the books and periodicals referred to in this list may be found at the Public Library, Eighth and K streets northwest. The residue may be found in the Prints Division, Library of Congress, or in the artists' file in the library of the Corcoran Gallery of Art, which may be consulted by students specially interested. 




\section{THE PUBLIC LIBRARY}

OF THE

\section{DISTRIGT OF COLUMBIA}

REFERENCE LIST No. 14

Contemporary American Painters

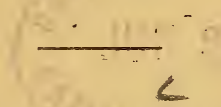

References to literature in books and periodicals on many of the exhibitors at the sixth biennial exhibition of contemporary American oil paintings, Corcoran Gallery of ... * Art, December, 1916, and-January, 1917.

MATIONAL COLLECTION OF FINE ART

Issued with the cooperation of

The Corcoran Gallery of Art

\section{THE PUBLIC LIBRARY}

WASHINGTON, D. C.

December, 1916 


\section{CONTEMPORARY \\ AMERICAN PAINTERS}

Siost of the painters exhibiting are represented in "American Art Annual" and "Who's Who in Art." When represented in that reference work or by slight mentio , in any of the other books given at the end of this list no specific notes to such references are made in the following list.

Under each exhibitor (so far as he has been written about) references are given to chapters in books (list.d pp. 15-16) and to magazine articles. Authors of such articles are not given and titles are omitted unless they point to special phases of the work of the artist treated.

\section{HUGO BALLIN}

Critic 47:497' (Dec., '05).

\section{GIFFORD BEAL}

Int. Studio 44:sup. 29-30 (Aug., '11).

- th CECILIA BEAUX

Books: Clement, Hartmann, Isham.

Magazines: Cent. 80:581-87 (Aug., '10).

Int. $\aleph^{2}$ udio 8:215-22 (Oct., '99).

Int. Studio 41 :sup. 3-10 (July, '10).

Scrib. Mag. $22: 477-85$ (Oct., '97).

\section{GEORGE BELLOWS}

Arts and Dec. 4:870-73 (Aug., '14). Art and Prog. 3:679-82 (Aug., '12). Int. Studio $56: 242-4$ (Oct., '15). 


\section{FRANK W. BENSON}

Book: Isham.

Magaxines Arts and Dec. 1:195-97 (Mar., '11). Harper 119:105-14 (June, '09). Int. Studio 35 sup. 99-10s (Oct., '08).

\section{CHARLES BITTINGER}

Int. Studio 59 :sup. 41-4 (Aug., '16).

RALPH ALBERT BLAKELOCK

Int. Studio 59 :sup. 19 (July, '16).

Lit. Digest 52:1147-8 (Apr. 22, '16).

Nation 102:473 (May 4, '16).

\section{LESTER D. BORONDA}

Int. Studio 54:sup. 180-2 (Feb., '15).

\section{HUGH H. BRECKENRIDGE}

Int. Studio $3 \%$ :sup. 34-6 (Mar., '09).

\section{GEORGE ELMER BROWNE}

1at. Studio 86:286-91 (Feb., '09).

\section{GEORGE DE FOREST BRUSH}

Books: Caffin-Am. masters, Hartmann, Isham.

Magazines: Cent. 21:600,638 (Feh., '92). Critic 47:134-8j (Aug., '05). Int. Studio 34:sup. 47-56 (Apr.) '08). 


\section{MARY CASSATT}

Books Clement, Isham.

Magazines: Arts and Dec. 3:265-7 (June, '13). Craftsman 19:540-6 (Mar., '11).

Int. Studio 27 :sup. 1-7 (Nov., '05). Scrib. M. 19:353-61 (Mar., '96).

World Today 21:1659-62 (Jen., '12).

\section{WILLIAM M. CHASE}

Books Caffin-Story of American painting, II a r t m a n, Isham, McSpadden, Muther.

Magarines Am. Mag. of Art 8:45-53 (Dec, '16).

Arts and Dec. 2:285-7 (June, '12). Craftsman 18:33-45 (A pr., '10). Int. Studio 39 :sup. 29-36 (Dec,s '09).

\section{CHARLOTTE B. COMAN}

Book: Clement.

\section{E. IRVING COUSE}

Craftsman 18:619-25 (Sept., '10).

\section{KENYON COX}

Book: Caffin-Story of American painting. Magazines: Arts and Dec. 6:295 (Apr., '16). Int. Studio 32:sup. 3-12 (July, 'OF).

\section{RANDALL DAVEY}

A m. Mag. of Art 7:314-7 (June, '16). 


\section{ARTHUR B. DAVIES}

Books: Hartmann, Huneker, Isham.

Magazines: Art and Archæol. 4:169-77 (Sept,, '16).

Forum 51:770-2 (May, '14).

\section{CHARLES H. DAVIS}

Book: Hartmann.

Magazine: New Eng. Mag. n. s. 27:423-87 (Dec., '02).

\section{HENRY GOLDEN DEARTH}

Cent. 70:157 (May, '05).

\section{JOSEPH DE CAMP}

Art and Prog. 4:919-25 (Apr., '13). A rts and Dec. 1:248-50 (A pr., '11). New Eng. Mag. n. s. 39:238-39 (Oct., '08).

\section{THOMAS W. DEWING}

Books: Caffin-Story of American painting, Hartmann, Isham.

Magazines: Critic 48:418-19 (May, '06). Harper $91: 165-70$ (July, '95). Harper 116:714-24, (Apr., '08).

\section{PAUL DOUGHERTY}

Int. Studio 36:sup. 8-4 (Nov., '08).

\section{GUY PENE DUBOIS}

Arts and Dec. 4:13-16 (Nov., '13). 


\section{FRANK DUVENECK}

Books: Caffin-Story of American painting, Isham.

Magazines: Arts and Dec. 1:382-84 (July, '11). Scrib. Mag. 58:643-46 (Nov., '15).

\section{LYDIA FIELD EMMET}

Book: Clement.

Magarine: Arts and Dec. 2:415-18 (Oct, '12).

\section{BEN FOSTER}

Outlook 68:58-9 (May, '01).

\section{FREDERICK C. FRIESCKE}

Art and Prog. 3:747-49 (Oct., '12). A rts and Dec. 3:13-15 (Nov., '12). Int. Studio 53:259-64 (Oct., '14). Scrib. Mag. 58:(64:-6 (Nov., '15).

\section{EDMUND GARRETT}

Am. Mag. of Art 7:493-95 (Oct., '16).

\section{LEON GASPARD}

Scrib. Mag. 59:281-88 (Mar., '16).

\section{WILLIAM J. GLACKENS}

Arts and Dec. 4:404-6 (Sept., '14).

Arts and Dec. 7:85 (Dec., '16).

Int. Studio 40:sup. 68 (May, '10).

\section{ANNE GOLDTHWAITE}

Int. Studio 59:sup. 8-8 (July, '16). 


\section{ALBERT L. GROLL}

Craftsman 9:826-36 (Mar., '06).

Int. Studio 28:sup. 78-82 (May, '06).

Int. Studio 29:270-71 (Sept., '06).

\section{PHILIP L. HALE}

Worid Today 14:59-67 (Jan., '08).

\section{CHILDE HASSAM}

Books: Caffin-Story of American painting, Hartmann, McSpadden.

Magazines: Int. Studio 45̃:sup. 29-33 (Dec., '11).

Int. Studio 57:sup. 82-6 (Jan., '16).

\section{CHARLES W. HAWTHORNE}

Art and Prog. 4:821-5 (Jan., '13).

Int. Studio 26:261-4. (Sept., '05).

Int. Studio 37 :sup. 65-8 (May, '09).

\section{ROBERT HENRI}

Book: Hartmann.

Magazines: Am. Mag. of Art 7:478-9 (Oct., '16).

Arts and Dec. 2:213-15 (Apr., '12). Ind. 64: pt. 2:1427-32 (June 25, '08).

Int. Studio 56:239-41 (Oct., '15).

\section{JOHN C. JOHANSEN}

Int. Studio 26:264-67 (Sept., '05).

Int. Studio 42 :sup. 3-6 (Nov., '10). 


\section{W. SERGEANT KENDALL}

Harper 117:568-77 (Sept., '08).

\section{LOUIS KRONBERG}

Int. Studio 28:74-5 (Mar., '06). New Eng. Mag. n. s. 38:499 (June, '08).

\section{ERNEST LAWSON}

Arts and Dec. 6:505-7 (Sept., '16). Cur. Lit. 42:406-9 (A pr., '07). Int. Studio 59 :sup. 13-15 (July, '16).

HAYLEY LEVER

Int. Studio 52 :sup. 92-3 (May, '14).

\section{JONAS LIE}

Craftsman 13:135-9 (Nov., '07). Cur. Lit. 52:222-4 (Feb., '12). Int. Studio 51 sup. 192-4 (Feb., "14).

\section{PHILIP LITTLE}

Art and Prog. 5:19-21 (Nov., '13).

\section{GEORGE B. LUKS}

A rts and Dec. 4:335-37 (July, 14). Craftsman 12:598-607 (Sept., '07). Int. Studio $56: 241-2$ (Oct., '15). 


\section{JEAN MCLANE (MRS. JOHN C. JOHANSEN)}

Art and Prog. 3:612 (June, '12). A rts and Dec. 3:299-301 (July, '13). Harper 110:291-3 (Jan., '09).

\section{H. MEAKIN}

Int. Studio 33:sup. 3-12 (Nor., '07).

\section{GARI MELCHERS}

Books: Caffin-Story of American painting, Muther.

Magazines: Int. Studio 31:sup. 11-18 (Mar.y '07).

Int. Studio 48:sup. 27-30 (Dec., '12).

Harper 114:430-9 (Feb., '07).

World's Work 15:10092-105 (Apr., '08).

\section{WILLARD L. METGALF}

Booklover's 6:509-11 (Oct., '05).

Cent. 77:155 (Nov., '08).

\section{RICHARD E. MILLER}

Int. Studio 43:268-73 (June, '11).

\section{F. LUIS MORA}

Craftsman 17:402-8 (Jan., '10). Harper 123:888 (Nov., '11). 


\section{JEROME MYERS}

Art and Prog. 5:89-94 (Jan., '14). Int. Studio 57 :sup. 125-8 (Feb., '16).

\section{WILLIAM M. PAXTON}

Int. Studio 39 :sup. 46-8 (Dec., '09). World 'loday 9:1210-14 (Nov., '05).

\section{HENRY R. POORE}

Int. Studio 37 :sup. 20-4 (Mar., '69). New Eng. Mag. n. s. 89:605 (J an., '09).

MAURICE B. PRENDERGAST World Today 19:716-19 (July, '10).

\section{EDWARD W. REDFIELD}

A rena 86:20-6 (July, '06). Arts and Dec. 6:135 (Jan., '16). Int. Studio 41 :sup. 29-36 (Aug., '10).

\section{ROBERT REID}

Book: Hartmann.

Magazines: Arts and Dec. 2:13-15, 34 (Nor,, '11).

Booklover's 6:738-42 (Dec., '05). Craftsman 7:699-712 (Mar., '05). Int. Studio 86 :sup. 113-22 (Feb., '09).

\section{HENRY REUTERDAHL}

Everybody's 32:838-52 (Mar., '15). 


\section{WILLIAM S. ROBINSON}

Int. Studio 89:10-11 (Nov., '09).

\section{JOHN SINGER SARGENT}

Books: Caffin-American masters, Cortissoz, D'Auvers, Hartmann, Isham, McSpadden, Muther, Sargent.

Magazines: Art and Prog. 3:742-5 (Oct., '12). Forum 54:232-6 (Aug., '15). Int. Studio 10:3-21 (Mar., '00). Int. Studio 10:107-19 (A pr., '00). Scrib. Mag. 84:515-32 (Nov., '03). World's Work 7:4:099-118 (Nov. '03).

\section{W. ELMER SCHOFIELD}

Arts and Dec. 1:473-5 (Oct., '11). Int. Studio 48:280-6 (Feb., '13).

\section{JOHN SLOAN}

Arts and Dec. 5:390-91 (Aug., '15). Arts and Dec. 6:237 (Mar., '16). Craftsman 15:559-64 (Feb., '09). Haxp. Y. 58:20-1 (Nov. 22, '13).

\section{EUGENE E. SPEICHER}

Int. Studio 46 :sup. 19-20 (Mar., '12).

\section{GARDNER SYMONS}

Outlook 105:881-7 (Dec. 27, '13). 


\section{EDMUND C. TARBELL}

Book: Hartmann.

Magazines: Arts and Dec. 2:129-31 (Feb., '12).

Harper 117:65- 74 (June, '08).

Int. Studio 32:sup. 75-87 (Sept., '07).

World Today 11:1077-85 (Oct., '06).

\section{ALLEN TUCKER}

Int. Studio 52:19-20 (Mar., '14).

\section{DOUGLAS VOLK}

Cent. 68:654-5 (Aug., '04).

\section{ROBERT VONNAH}

Books: Hartmann, Muther.

Magazines: Art and Prog. 4:999-1002 (June, '13).

Art and Dec. 2:381-3 (Sept., '12). Int. Studio 54:sup. 48-52 (Dec., '14).

\section{HORATIO WALKER}

Book: Caffin-American masters.

Magazines: Arts and Dec. 1:63-5, 88 (Dec., '10).

Canad. Mag. 18:496-500 (Apr., '02). Craftsman 14:138 (May, '08). Harper 117:947 (Nov., '08). 


\section{MARTHA WALTER}

Arts and Dec. 1:303-5 (May, '11).

Int. Studio 52 :sup. 42-5 (A pr., '14).

\section{FREDERICK J. WAUGH}

Arts and Dec. 1:111-18 (Jan., '11).

Int. Studio $51: 273-8$ (Feb., '14).

\section{J. ALDEN WEIR}

Books: Hartmann, Isham, McSpadden.

Magazines: Arts and Dec. 2:55-7 (Dec., '11). Outlook 110:120 (May 19, '15). Scrib. Mag. 59:129-32 (Jan, '16).

IRVING R. WILES

Arts and Dec. 1:402-4 (Aug., '11).

Craftsman 18:347-53 (June, '10).

\section{FREDERICK BALLARD WILLIAMS}

Int. Studio 42:sup. 53-60 (Jan., '11).

\section{CHARLES H. WOODBURY}

Art and Prog. 4:761-6 (Nov., '12). 


\section{COLLECTIVE ARTICLES}

The following magazine articles group several painters together, comparing them with American and European artists not exhibiting; some of them discuss certain later movements, tendencies and revoits against the established standards. They help to explain the great diversity of the work shown.

This transitional age in art: J. W. Alexander, Is our art distinctively American? E. H. Blashfield, The painting of today; E. L. Blumenschein, The painting of tomorrow; Walter Pach, The point of view of the "moderns"; Jay Hambridge and Gove Hambridge, The ancestry of Cubism. Cent. 87:825-75 (Apr., '14).

Special exhibition numbers of Arts and Decoration: Arthur B. Davies, Explanatory statement; G. P. DuBois, Spirit and chronology of the modern movement; John Quinn, Modern art from a layman's point oî view; W. J. Glackens, American section-the national art; F. J. Gregg, Attitude of the Americans; W. M. Fisher, Sculpture at the exhibition; J. Davidson, The extremists-an interview; M. Dodge, Speculations or post-impressionism in prose. Arts and Decoration 3:172 (Mar., '18).

Some American portrait painters: Sargent, Eakins, Duveneck, Thayer, Brush, Henri, Alexander, Chase, Weir and Beaux. C. H. Caffin. Critic 44:21-45 (Jan., '04). 
Some new American painters in Paris: Hubbell, Frieseke and McLane. C. H. Cafin. Harper $118: 284-93$ (J an., '09).

American painting today: Sargent, Chase, Thayer and Weir. Ernest Knaufft. Rev. of Rer. 36:693-702 (Dec., '07).

Some American marine painters: Carlsen, Dougherty, Waugh, Woodbury. Anna SeatonSchmidt. Art and Prog. 2:2-8 (Nov., '10).

\section{BOOKS}

Caffin, C. H. American masters of painting. 1918. W10-9C112.

Caffin, C. H. Story of American painting. 1907. WP83-C11.

Clement, Mrs. C. E. Women in the fine arts. 1904. W10-9C59w.

Cortissoz, Royal. Art and common sense. 1918. W-C817.

D'Anvers, N. Representative painters of the nineteenth century. 1899. W10-9D238. Reference.

Hartmann, Sadakichi. History of American art. 2 v. 1902. W83-H255.

Honeker, James. Pathos of distance. 1918. Y-H89sp. 
Isham, Samuel. History of American painting. 1907. WP-IsE26h.

McSpadden, J. W. Famous painters of America. 1916. W10-9M246.

Muther, Richard. History of modern painting (v. 4, America). 1907. WP-4.M984.

Sargent, J. S. Work of Sargent, with intro. by Mrs. Meynell. 1903. WP-Sa743. Reference.

Who $s$ Who in Art. (In American art annual, 1915) W-7Am. Reference.

All of the books and periodicals referred to in this list may be found at the central building of the Public Library, Eighth and K Streets N. W.

The Public Library has published several reference lists on the history of painting and on various phases of the fine arts. The assistants in the reference room are always glad to help students of art history or criticism to locate needed material.

P. L. 487. $5 M-15-12=^{\circ} 16$. 


\section{W31 012 \\ $919 \mathrm{THE}$ \\ PUBLIC LIBRARY \\ OF THE}

DISTRICT OF COLUMBIA

REFERENCE LIST No. 15

Contemporary American Painters

References to literature in books and pexiodicals on many of the exhibitors at the Seventh Exhibition of Contemporary American Oil Paintings, Corcoran Gallery of Art, December, 1919, and January, 1920.

\section{NATIOHAL COULCTHOH OF FINE ARTS}

- Issued with the cooperation of

The Corcoran Gallery of Art

THE PUBLIC LIBRARY WASHINGTON, D. C.

December, 1919 


\section{CONTEMPORARY AMERICAN PAINTERS}

Brief biographical information in regard to most of the painters exhibiting is given in "Who's Who in Art, 1917". When an artist is represented in that reference work or by slight mention in any of the other books included in the list, no specific note is made.

Under each exhibitor (so far as he has been written about), references are given to chapters in books (listed pp. 18-19) and to magazine articles. The items marked with a star (*) are not in the Public Library. All other books and periodicals referred to in this list may be found in the Public Library, Eighth and $\mathrm{K}$ Streets, N. W.

\section{WILLIAM AUERBACH-LEVY}

Survey $32: 193$ (May 16, '14).

\section{FREDERIC CLAY BARTLETT} *Sketch Book 5 :247 (Feb., '06).

\section{GIFFORD BEAL}

Int. Studio 44 : sup. 29-30 (Aug., '11). Touchstone $5: 240-3$ (June, '19).

\section{CECILIA BEAUX}

Books: Clement, Hartmann, Isham.

Magazines: Art World 3: 489-90 (Mar., '18). Century 80:581-87 (Aug., '10). Int. Studio $8: 215-22$ (Oct., '99). Int. Studio 41 : sup. 3-10 (July, '10).

Scribner's $22: 477-85$ (Oct., '97). 


\section{GEORGE BELLOWS}

Art and Prog. 3:679-82 (Aug., '12). Arts and Dec. 4:370-73 (Aug., '14). Int. Studio 56:242-4 (Oct., '15). Touchstone 1:269-75 (July, '17).

\section{GERRIT BENEKER}

Red Cross Mag. $14: 18-22$ + (Nov., '19).

\section{FRANK W. BENSON}

Book: Isham.

Magazines: Arts and Dec. 1:195-97 (Mar., '11).

Country Life 36:53-5 (Oct., '19). Harper's 119:105-14 (June, '09). Harper's 138:826 (May, '19).

Int. Studio 35 : sup. 99-104 (Oct., '08).

\section{ADOLPHE BLONDHEIM}

Canad. Mag. 52:1058-61 (Apr., '19).

\section{HUGH H. BRECKENRIDGE}

Int. Studio 37: sup. 34-6 (Mar., '09).

\section{BENJAMIN C. BROWN}

Int. Studio $43: 121-31$ (Apr., '11).

\section{GEORGE ELMER BROWNE}

Int. Studio 36:286-92 (Feb., '09). 


\section{GEORGE DE FOREST BRUSH}

Books: Caffin-American masters, Hartmann, Isham.

Magazines: Art World 2:8-9 (Apr., '17).

Critic 47:134-35 (Aug., '05). Int. Studio 34 : sup. 47-56 (Apr., '08).

\section{HOWARD RUSSELL BUTLER}

Art World 2:310-12 (July, '17).

\section{EMIL CARLSEN}

Int. Studio 61: sup. 105-10 (June, '17).

\section{JOHN F. CARLSON}

*Fine Arts J. 35:203-6 (Mar., '17).

\section{MARY CASSATT}

Books: Cary - Artists past and present (W10-9C258), Clement, Isham.

Magazines: Arts and Dec. 3:265-7 (June, '13).

Craftsman 19:540-6 (Mar., '11). Cur. Opinion 62:426-7 (June, '17).

Int. Studio 27: sup. 1-7 (Nov., '05).

Print Col. Q. 6:396-409 (Dec., '16).

Scribner's 19 :353-61 (Mar., '96). World Today 21:1659-62 (Jan., '12). 


\section{SIDNEY M. CHASE}

Scribner's 43:641-55 (June, '08).

Scribner's $46: 1-11$ (July, '09).

Scribner's 48:554-63 (Nov., '10).

Scribner's 52:193-203 (Aug., '12).

Scribner's $53: 531-43$ (May,' 13 ).

\section{ELIOT CLARK}

Country Life 35:45-7 (Feb., '19).

\section{WILLIAM BAXTER CLOSSON}

Print. Art 31:118-22 (Apr., '18).

\section{COLIN C. COOPER}

Art and Prog. 3:601 (June, '12).

Art World $3: 2,5$ (Oct., '17).

*Booklover's 5:327 (Mar., '05).

*Fine Arts J. (Oct., '03).

\section{E. IRVING COUSE}

Craftsman 18:619-25 (Sept., '10).

BRUCE CRANE

*Brush and Pencil 11:1-10 (Oct., '02).

\section{CHARLES C. CURRAN}

Critic 48:38-9 (Jan., '06).

\section{ARTHUR B. DAVIES}

Books: Hartmann, Huneker-Pathos of distance (Y-H894p) Isham. 
Magazines: Art and Archaeol. 4:169-77 (Sept., '16). *Art in Amer. 6:295-9 (Oct., '18). Arts and Dec. $8: 34,36-7$ (Nov., '17).

Cur. Opinion 64:203-5 (Mar., '18).

Forum 51: 770-2 (May, '14).

Lit. Dig. $56: 23-4$ (Jan. 26, '18). New Repub. 13:338 (Jan. 19, '18).

\section{CHARLES H. DAVIS}

Book: Hartmann.

Magazine: New Eng. Mag. n. s. 27: 423-37 (Dec., '02).

\section{JOSEPH DE CAMP}

Art and Prog. 4:919-25 (Apr., '13). Arts and Dec. 1:248-50 (Apr., '11).

New Eng. Mag. n. s. $39: 238-39$ (Oct., '08).

\section{LOUIS P. DESSAR}

*Brush and Pencil 5:97 (Dec., '99). Harper's $122: 282$ (Jan., '11).

\section{CHARLES M. DEWEY}

Harper's $114: 444$ (Feb., '07).

\section{THOMAS W. DEWING}

Books: Caffin-Story of American painting, Hartmann, Isham.

Magazines: Art World 3:188-9 (Dec., '17). Critic 48:418-19 (May, '06). Harper's 91:165-70 (July, '95). Harper's 116:714-24 (Apr., '08). 


\section{PAUL DOUGHERTY}

Int. Studio 36: sup. 3-4 (Nov., '08).

\section{FRANK V. DUDLEY}

*Fine Arts J. 36:19-26 (June, '18).

\section{FRANK V. DuMOND}

Art and Prog. 4:772 (Nov., '12).

\section{BEN FOSTER}

"Fine Arts J. 34:176-80 (Apr., '16). Outlook 68:58-9 (May, '01).

\section{FREDERICK C. FRIESEKE}

Art and Prog. 3:747-49 (Oct., '12). Arts and Dec. 3:13-15 (Nov., '12). Int. Studio 53:259-64 (Oct.,' 14 ). Scribner's 58:643-6 (Nov., '15).

\section{MAURICE FROMKES}

Century 77:958 (Apr., '09).

\section{DANIEL GARBER}

*Maitres Contemporains 59:10 (1912). 


\section{WILLIAM J. GLACKENS}

Amer. Mag. of Art 7:261-3 (May, '16).

Arts and Dec. 4:404-6 (Sept., '14).

Arts and Dec. 7:85 (Dec., '16).

Int. Studio 40 : sup. 68 (May, '10).

\section{WALTER GRIFFIN}

Int. Studio 62: sup. 45-8 (Aug., '17).

\section{ALBERT L. GROLL}

Craftman $9: 826$-36 (Mar., '06).

Int. Studio 28: sup. 78-82 (May, '06).

Int. Studio $29: 270-71$ (Sept., '06).

Int. Studio 64: sup. 105-10 (June, '18).

PHILIP L. HALE

World Today 14:59-67 (Jan., '08).

\section{JOHN MCCLURE HAMILTON}

Book: Isham.

Magazine: Scribner's 27:733-38 (June, '00).

\section{BIRGE HARRISON}

Art and Prog. 3:379-83 (Nov., '11).

Craftsman 13:397-99 (Jan., '05).

Int. Studio 44: sup. 3-5 (July, '11).

Scribner's $42: 576-84$ (Nov., '07).

\section{CHILDE HASSAM}

Books: Caffin-Story of American painting, Hartmann, McSpadden. 
Magazines. Art and Prog. 2:137-39 (Mar., 11).

Int. Studio 45: sup. 29-33 (Dec., '11).

Int. Studio 57: sup. 83-6 (Jan., '16).

Nation 101:698-9 (Dec. 9, '15). Touchstone $5: 272-80$ (July, '19).

\section{CHARLES W. HAWTHORNE}

Art and Prog. 4:821-5 (Jan., '13).

Int. Studio $26: 261-4$ (Sept., '05).

Int. Studio 37: sup. 65-8 (May, '09).

Int. Studio 61: sup. 19-24 (Mar., '17).

\section{ROBERT HENRI}

Book: Hartmann.

Magazines: Amer. Mag. of Art 7:473-9 (Oct., '16).

Arts and Dec. 2:213-15 (Apr., '12).

Craftsman $27: 459-69$ (Feb., '15). Ind. 64: pt. 2:1427-32 (June 25, '08).

Int. Studio $30: 182-3$ (Dec., '06). Int. Studio $56: 239-41$ (Oct., '15). Touchstone 5:212-19 (June,'19).

\section{WILLIAM H. HOLMES}

Art and Prog. 6:11-14 (Nov., '14).

\section{JAMES R. HOPKINS}

Int. Studio 63: sup. 113-19 (Feb., '18). 


\section{WILLIAM H. HOWE}

Art World 3:3-6 (Oct., '17).

\section{E. T. HURLEY}

Int. Studio 29 : sup. 63-71 (Sept., '06).

\section{FRANK TOWNSEND HUTCHENS}

*Fine Arts J. 34:124-7 (Mar., '16).

Int. Studio 47:160-1, (Aug., '12).

\section{JOHN C. JOHANSEN}

Int. Studio 26 :264-67 (Sept., '05). Int. Studio 42 : sup. 3-6 (Nov., '10).

\section{W. SERGEANT KENDALL}

Harper's 117:568-77 (Sept., '08).

\section{LOUIS KRONBERG}

Int. Studio 28:74-5 (Mar., '06). New Eng. Mag. n. s. 38:499 (June, '08).

\section{ERNEST LAWSON}

Amer. Mag. of Art 8:257-63 (May, '17). Arts and Dec. 6:505-7 (Sept., '16). Arts and Dec. 10:257-8 (Mar., '19). Cur. Lit. 42:406-9 (Apr., '07). Int. Studio 59: sup. 13-15 (July, '16).

\section{HAYLEY LEVER}

Int. Studio 52: sup. 92-3 (May, '14). 


\section{JONAS LIE}

Bul. Pan. Amer. Union 38:679-83 (May, '14). Country Life 36:55-7 (July, '19).

Craftsman $13: 135-9$ (Nov., '07).

Cur. Lit. 52 :222-4 (Feb., '12).

Int. Studio 51: sup. 192-4 (Feb., '14).

\section{PHILIP LITTLE}

Art and Prog. 5:19-21 (Nov., '13).

GEORGE B. LUKS

Arts and Dec. 4:335-37 (July, '14).

Craftsman 12:598-607 (Sept., '07).

Int. Studio 56:241-2 (Oct., '15).

WALTER McEWEN

*Maitres Contemporains 46:8 (1908).

\section{JEAN McLANE (MRS. JOHN C. JOHANSEN)}

Art and Prog. 3:612 (June, '12). Arts and Dec. 3:299-301 (July, '13). Harper's 118:291-3 (Jan., '09).

\section{GARI MELCHERS}

Books: Caffin-Story of American painting, Muther.

Magazines: Harper's 114:430-9 (Feb., '07). Int. Studio 31 : sup. 11-18 (Mar., 07).

Int. Studio 48: sup. 27-30 (Dec., '12).

W or ld's Work $15: 10092-105$ (Apr., '08). 


\section{WILLARD L. METCALF}

*Booklover's 6:509-11 (Oct., '05). Century $77: 155$ (Nov., '08).

New Eng. Mag. n. s. 39:374-75 (Nov., '08).

\section{RICHARD E. MILLER}

Int. Studio $43: 263-73$ (June, '11).

\section{F. LUIS MORA}

Country Life 33:57-9 (Feb., '18). Craftsman 17:402-08 (Jan., '10). Harper's 123:888 (Nov., '11).

\section{HERMANN D. MURPHY}

*Brush and Pencil 5:49 (Nov., '99). *Sketchbook 6:303 (Nov., '07).

\section{J. FRANCIS MURPHY}

*Art in Amer. $6: 163-8$ (Apr., '18). Arts and Dec. 3:191-93 (Apr.,'13). Int. Studio 53: sup. 3-10 (July, '14). Scribner's 61:127-30 (Jan., '17).

\section{JEROME MYERS}

Amer. Mag. of Art 8:481-5 (Oct., '17). Art and Prog. 5:89-94 (Jan., '14). Arts and Dec. 10:257, 259 (Mar., '19). Craftsman $29: 25-32$ (Oct., '15). Int. Studio 57: sup. 125-8 (Feb., '16). Touchstone 5:396-9 (Aug., '19).

\section{JULES PAGES}

Art and Prog. 5 :385-7 (Sept., '14). *Fine Arts J. 31:511-12 (Oct., '14). 


\section{LAWTON S. PARKER}

Collier's $52: 23$ (Jan. 31, '14).

Int. Studio 57: sup. 37-43 (Dec., '15).

\section{WILLIAM M. PAXTON}

Int. Studio 39: sup. 46-8 (Dec., '09). World Today $9: 1210-14$ (Nov., '05).

VAN DEARING PERRINE

Craftsman 12:486-96 (Aug., '07).

Cur. Lit. 41:407-10 (Oct., '06).

\section{CHARLES A. PLATT}

Book: *Monograph on the work of Charles A. Platt, with introd. by Royal Cortissoz. 1913.

Magazines: Arch. Rec. $15: 181-244$ (Mar., '04).

Arch. Rec. 36:81-97 (Aug., '14).

MAURICE B. PRENDERGAST

World Today 19:716-19 (July, '10).

\section{EDWARD W. REDFIELD}

Arena 36:20-6 (July, '06).

Arts and Dec. 6:135 (Jan., '16).

Country Life 13:194-97 (Dec., '07).

Int. Studio 41: sup. 29-36 (Aug., '10).

Book: Hartmann.

\section{ROBERT REID}

Magazines: Arts and Dec. $2: 13-15,34$ (Nov., '11).

Booklover's $6: 738-42$ (Dec., '05). Craftsman 7:699-712 (Mar., '05).

Int. Studio 36: sup. 113-22 (Feb., '09). 


\section{HENRY REUTERDAHL}

Everybody's $32: 338-52$ (Mar., '15).

Scribner's $55: 307-10$ (Mar., '14).

\section{LOUIS RITMAN}

Int. Studio 67: sup. 62-4 (Apr., '19).

\section{WILLIAM RITSCHEL}

*Fine Arts J. $34: 275-80$ (June, '16).

ELIZABETH WENTWORTH ROBERTS

New Eng. Mag. n. s. $24: 487-93$ (July, '01).

\section{WILLIAM S. ROBINSON}

Int. Studio $39: 10-11$ (Nov., '09).

\section{ERNEST D. ROTH}

Amer. Mag. of Art 9 :358-61 (July, '18).

Print Coll. Q. 1:442-56 (Oct., '11).

\section{CHAUNCEY F. RYDER}

*Art Review $22: 1$ (Sept., '11).

\section{JOHN SINGER SARGENT}

Books: Brinton - Modern artists (W10-

9B774 Reference), Caffin-American masters, Cortissoz-Art and common sense (W-C817), CoxArtist and public (W-C838), Hartmann, Isham, McSpadden, Muther, Sargent-Work of Sargent, with introd. by Mrs. Meynell (WP-Sa743 Reference). 
Magazines: Amer. Mag. of Art 8:129-36 (Jan., '17).

Art and Prog. 3:742-5 (Oct., '12).

Arts and Dec. 7:194-7 (Feb., 17).

Forum $54: 232-6$ (Aug., '15).

Int. Studio 10:3-21 (Mar., '00). Int. Studio 10:107-19 (Apr., '00).

Scribner's $34: 515-32$ (Nov., '03). World's Work 7:4099-118 (Nov., '03).

W. ELMER SCHOFIELD

Arts and Dec. 1:473-5 (Oct., '11).

Int. Studio 48:280-6 (Feb., '13).

\section{EDWARD SIMMONS}

Book: Isham.

Magazine: Cur. Lit. 28:40 (Apr., '00).

\section{EUGENE E. SPEICHER}

Int. Studio 46: sup. 19-20 (Mar., '12).

\section{MAURICE STERNE}

Cur. Opinion 59:425-7 (Dec., '15).

Int. Studio 46: sup. 3-13 (Mar., '12). Int. Studio 61: sup. 53-6 (Apr., '17).

\section{GARDNER SYMONS}

*Fine Arts J. 34 :557-65 (Nov., '16). Outlook $105: 881-7$ (Dec. 27, 13 ). 


\section{EDMUND C. TARBELL}

Book: Hartmann.

Magazines: Amer. Mag. of Art 9:217-28

$$
\text { (Apr.,'18). }
$$

Arts and Dec. 2:129-31 (Feb., '12).

Harper's 117:65-74 (June, '08). Int. Studio 32 : sup. 75-87 (Sept., '07).

World Today 11:1077-85 (Oct., '06).

\section{ABBOTT H. THAYER}

Books: Caffin-Story of American painting, D'Anvers-Representative painters of the nineteenth century (W109D238 Reference), Hartmann, Isham.

Magazines: Critic 46:423 (May, '05). Int. Studio $6: 247-54$ (1899). Int. Studio 33: sup. 81-87 (Jan., '08).

\section{HELEN M. TURNER}

Art World 3:490 (Mar., '18).

WÄLTER UFER

*Fine Arts J. $34: 221-6$ (May, '16).

\section{ELIHU VEDDER}

Books: Hartmann, Isham, McSpadden, Vedder-Digressions of V (W10V513). 
Magazines: Bookman 35:145-53 (Apr., '12). Int. Studio 35 : sup. 94 (Sept., '08).

Outlook 96:693-98 (Nov., 26, '10).

\section{DOUGLAS VOLK}

Art World 3:282-3 (Jan., '18). Century 68:654-5 (Aug., '04).

\section{ROBERT VONNOH}

Books: Hartmann, Muther.

Magazines: Art and Prog. 4:999-1002 (June, '13).

Arts and Dec. 2:381-3 (Sept., '12).

Int. Studio 54: sup. 48-52 (Dec., '14).

\section{HORATIO WALKER}

Book: Caffin-American masters.

Magazines: Arts and Dec. 1:63-5, 88 (Dec., '10).

Canad. Mag. 18:496-500 (Apr., '02).

Canad. Mag. 53 :21-9 (May, '19). Craftsman 14:138 (May, '08). Harper's 117:947 (Nov., '08). Touchstone $5: 482-5$ (Sept., '19).

\section{EVERETT WARNER}

Scribner's 46:280-92 (Sept., '09). 
FREDERICK J. WAUGH

Arts and Dec. $1: 111-13$ (Jan., '11).

Int. Studio 51:273-8 (Feb., '14).

Int. Studio 60: sup. 50-2 (Dec., '16).

\section{J. ALDEN WEIR}

Books: Hartmann, Isham, McSpadden.

Magazines: Amer. Mag. of Art 8:213-20 (Apr., '17).

Arts and Dec. 2:55-7 (Dec.,'11).

Harper's 131:246-7 (July, '15).

Outlook 110:120 (May 19, '15). Scribner's $59: 129-32$ (Jan., '16).

FREDERICK BALLARD WILLIAMS

Int. Studio $42:$ sup. 71-8 (Feb., '11).

\section{CHARLES H. WOODBURY}

Art and Prog. 4:761-6 (Nov., '12).

Int. Studio 42: sup. $71-78$ (Feb., '11).

CHARLES MORRIS YOUNG

Int. Studio 28:369-70 (June, '19).

\section{BOOKS}

\section{ART APPRECIATION}

Noyes, C. E. Gate of appreciation. 1907. W-N873g.

Sturgis, Russell. Appreciation of pictures. 1905. WP-St974ap.

Van Dylre, J. C. How to judge of a picture. 1889. WP-V283h.

Van Dyke, J. C. What is art? 1910. WPV289w. 


\section{AMERICAN PAINTERS AND PAINTING}

Caffin, C. H. American masters of painting. 1913. W10-9C112.

Caffin, C. H. Story of American painting. 1907. WP83-C11.

Clement, Mrs. C. $\boldsymbol{E}$. Women in the fine arts. 1904. W10-9C59w.

Hartmann, Sadakichi. History of American art. 2v. 1902. W83-H255.

Innes, Mary. Schools of painting. 1911. WP-In65s.

Isham, Samuel. History of American painting. 1907. WP-Is326h.

McSpadden, J. W. Famous painters of America. 1916. W10-9M246.

Muther, Richard. History of modern painting. v. 3. 1907. WP-9M984 Reference.

Who's Who in Art. (In American art annual, 1917). W-7AM. Reference.

\section{COLLECTIVE ARTICLES}

The following magazine articles group several painters together, comparing them with American and European artists not exhibiting; some of them discuss certain later movements, tendencies and revolts against the established standards. They help to explain the great diversity of the work shown.

This transitional age in art: J. W. Alexander, Is our art distinctively American? E. H. Blashfield, The painting of today; E. L. Blumenschein, The painting of tomorrow; Walter Pach, The point of view of the "moderns"; Jay Hambridge and Gove Hambridge, The an- 
cestry of Cubism. Century 87:825-75 (Apr., '14).

Special exhibition number of Arts and Decoration: Arthur B. Davies, Explanatory statement; G. P. DuBois, Spirit and chronology of the modern movement; John Quinn, Modern art from a layman's point of view; W. J. Giackens, American section-the national art; F. J. Gregg, Attitude of the Americans; W. M. Fisher, Sculpture at the exhibition; J. Davidson, The extremists-an interview; M. Dodge, Speculations or, post-impressionism in prose. Arts and Decoration 3:149 (Mar., '13).

Some American portrait painters: . Sargent, Eakins, Duveneck, Thayer, Brush, Henri, Alexander, Chase, Weir and Beaux. C. H. Caffin. Critic 44:31-45 (Jan., '04).

American painting today: Sargent, Chase, Thayer and Weir. Ernest Knauff. Rev. of Rev. 36:693-702 (Dec., '07).

Some American marine painters: Carlsen, Dougherty, Waugh, Woodbury. Anna SeatonSchmidt. Art and Prog. 2:3-8 (Nov., '10).

Recent tendencies in marine painting: Birge Harrison. Scribner's 49:469-77 (Apr., '11).

Painters of the Palisades: Bailey Millard. Bookman 35:256-65 (May, '12).

The Public Library has published several reference lists on the history of painting and on various phases of the fine arts which are still available. The assistants in the reference room are always glad to help students of art history or criticism to locate needed material. 


\section{CONTEMPORARY AMERICAN PAINTERS}

Brief biographical information in regard to most of the painters exhibiting is given in 'Who's Who in Art, 1919". When an artist is represented in that reference work or by slight mention in any of the other books included in the list, no specific note is made.

Under each exhibitor (so far as he has been written about), references are given to chapters in books (listed pp. 16-17) and to magazine articles. The items marked with a star $\left(^{*}\right)$ are not in the Public Library. All other books and periodicals referred to in this list may be found in the Public Library, Eighth and K Streets, N. W.

\section{MAXWELL ARMFIELD}

Int. Studio 30:61-2 (Nov.,'’06).

Int. Studio 60: sup. 28-30 (Nov., '16).

Int. Studio 61:114-19 (May, '17).

\section{WILLIAM AUERBACH-LEVY}

Survey 32:193 (May 16, '14).

\section{CECELIA BEAUX}

Books: Clement, Hartmann, Isham.

Magazines: Art World 3:489-90 (Mar., '18).

Century 80:581-87 (Aug., '10).

Independent 103:382-4 (Sept. 25, '20). Int. Studio 8:215-22 (Oct., '99).

Int. Studio 41: sup. 3-10 (July, '10). Scribner's 22:477-85 (Oct., '97).

\section{GEORGE BELLOWS}

Art and Prog. 3:679-82 (Aug., '12). Arts and Dec. 4:370-73 (Aug., '14). 
Arts and Dec. 15:158-9 (July, '21).

Int. Studio 56:242-4 (Oct., '15).

Int. Studio 71: sup. 21-7 (Aug., '20).

New Repub. 23:220-1 (July 21, '20).

*Touchstone 1:269-75 (July, '17).

\section{FRANK W. BENSON}

Book: Isham.

Magazines: Amer. Mag. of Art 9:309-316 (June, '18).

Amer. Mag. of Art 12:365-372 (Nov., '21).

Arts and Dec. 1:195-97 (Mar., '11).

Harper's 119:105-14 (June, '09).

Harper's 138: 826 (May, '19).

Int. Studio 35: sup. 99-104 (Oct., '08).

Int. Studio 74:95-100 (Oct., '21).

\section{THERESA F. BERNSTEIN}

Int. Studio 66: sup. 97-102 (Feb., '19).

\section{CHARLES BITTINGER}

Int. Studio 59: sup. 41-4 (Aug., '16).

LESTER D. BORONDA

Int. Studio 54: sup. 130-2 (Feb., '15).

\section{HUGH H. BRECKENRIDGE "}

Amer. Mag. of Art 7:85-6 (Jan., '16).

Int. Studio 37: sup. 34-6 (Mar.,' '09).

\section{GEORGE ELMER BROWNE}

Int. Studio 36:286-92 (Feb., '09).

\section{GEORGE DE FOREST BRUSH}

Books: Caffin-American masters; Story of American painting, Hartmann, Isham. 
Magazines: Art World 2:8-9 (Apr., '17). Crític 47:134-35 (Aug., '05). Int. Studio 34: sup. 47-56 (Apr., '03).

\section{BRYSON BURROUGHS}

Book: Isham.

Magazine: Arts and Dec. 12:82-4 (Dec., '19).

\section{EMIL CARLSEN}

Int. Studio 61: sup. 105-10 (June, '17).

Scribner's 66:767-70 (Dec., '19).

*Touchstone 7:110-16 (May, '20)

\section{JOHN F. CARLSON}

*Fine Arts J. 35:203-6 (Mar., '17).

\section{MARY CASSATT}

Books: Cary-Artists past and present (W109 C258), Clement, Isham.

Magazines: Arts and Dec. 3:265-7 (June, '13). Craftsman 19:540-6 (Mar., '11). Cur. Opinion 62:426-7 (June, '17). Int. Studio 27: sup. 1-7 (Nov., '05). Print Col. Q. 6:396-409 (Dec.,' 16). Scribner's 19:353-61 (Mar., '96). World Today 21:1659-62 (Jan., '12).

\section{ADELAIDE COLE CHASE}

Book: Clement.

CLARENCE K. CHATTERTON

Art. and Archaeol. 5:289-93 (May, '17).

\section{CHARLOTTE B. COMAN}

Book: Clement. 


\section{E. IRVING COUSE}

Amer. Mag. of Art 11:400-5 (Sept., '20).

Century 99:369-76 (Jan., '20).

Craftsman 18:619-25 (Sept., '10).

\section{BRUCE CRANE}

*Brush and Pencil 11:1-10 (Oct., '02).

\section{ARTHUR B. DAVIES}

Books: Hartmann, Huneker-Pathos of distance (Y-H894p), Isham, Sherman-American painters of yesterday and today (W109Sh56).

Magazines: Art and Archaeol. 4:169-77 (Sept., '16).

*Art in Amer. 6:295-9 (Oct., '18).

Arts and Dec. 8:34, 36-7 (Nov., '17).

Cur. Opinion 64:203-5 (Mar., '18)

Forum 51:770-2 (May, '14).

Int. Studio 72: sup. 126-30 (Feb., '21).

Lit. Dig. 56:23-4 (Jan. 26, '18).

New Repub. 13:338 (Jan. 19, '18).

*Touchstone 6:277-84 (Feb., '20).

CHARLES H. DAVIS

Book: Hartmann.

Magazine: New Eng. Mag. n. s. 27:423-37 (Dec., '02).

\section{JOSEPH DE CAMP}

Art and Prog. 4:919-25 (Apr., '13).

Arts and Dec. 1:248-50 (Apr., '11).

New Eng. Mag. n. s. 39:238-39 (Oct., '08).

\section{THOMAS W. DEWING}

Books: Caffin-Story of American painting, Hartmann, Isham. 
Magazines: Amer. Mag. of Art 10:302-3 (June, '19).

Art World 3:188-9 (Dec., '17).

Critic 48:418-19 (May, '06).

Harper's 91:165-70 (July, '95).

Harper's 116:714-24 (Apr., '08).

\section{PAUL DOUGHERTY}

Arts and Dec. 8:251-4 (Apr., '18).

Int. Studio 30:180-182 (Dec., '06).

Int. Studio 36: sup. 3-4 (Nov., '08).

Int. Studio 73: sup. 54-58 (Apr., '21).

\section{GUY PENE DU BOIS}

Arts and Dec. 4:13-16 (Nov. '13).

\section{FRANK V. DUDLEY}

*Fine Arts J. 36:19-26 (June, '18).

\section{LYDIA FIELD EMMET}

Book: Clement.

Magazine: Arts and Dec. 2:415-18 (Oct., '12).

\section{JOHN F. FOLINSBEE}

Int. Studio 73: sup. 38 (Apr., '21).

\section{BEN FOSTER}

*Fine Arts J. 34:176-80 (Apr., '16). Outlook 68:58-9 (May, '01).

\section{FREDERICK CARL FRIESEKE}

Art and Prog. 3:747-49 (Oct., '12). Arts and Dec. 3:13-15 (Nov., '12). Int. Studio 53:259-64 (Oct., '14). Scribner's 58:643-6 (Nov., '15). 


\section{MAURICE FROMKES}

Century 77:958 (Apr., '09).

\section{DANIEL GARBER}

*Maitres Contemporains 59:10 (1912).

\section{WILLIAM J. GLACKENS}

Amer. Mag. of Art 7:261-3 (May, '16).

Arts and Dec. 4:404-6 (Sept., '14).

Arts and Dec. 7:85 (Dec.,'16).

Arts and Dec. 14:103 (Dec., '20).

Int. Studio 40: sup. 68 (May, '10).

*Touchstone 7:191-9 (June, '20).

\section{WALTER GRIFFIN}

Int. Studio 62: sup. 45-8 (Aug., '17).

\section{ALBERT L. GROLL .}

Century 100:385-92 (July, '20). Craftsman 9:826-36 (Mar., '06). Int. Studio 28: sup. 78-82 (May, '06). Int. Studio 29:270-71 (Sept., '06). Int. Studio 64: sup. 105-10 (June '18).

\section{PHILIP L. HALE}

Amer. Mag. of Art 7:259-60 (May, '16). World Today 14:59-67 (Jan., '08).

\section{GEORGE HARDING}

Amer. Mag. of Art 10:451-456 (Oct., '19). Int. Studio 62: sup. 16-18 (July, '17).

\section{BIRGE HARRISON}

Art and Prog. 3:379-83 (Nov., '11). Craftsman 13:397-99 (Jan., '05). Int. Studio 44: sup. 3-5 (July, '11). Scribner's 42:576-84 (Nov., '07). 


\section{CHILDE HASSAM}

Books: Caffin-Story of American painting, Hartmann.

Magazines: Art and Prog. 2:137-39 (Mar., '11).

Int. Studio 45: sup. 29-30 (Dec., '11).

Int. Studio 57: sup. 83-6 (Jan., '16).

Nation 101:698-9 (Dec. 9, '15).

*Touchstone 5:272-80 (July, '19).

\section{CHARLES W. HAWTHORNE}

Art and Prog. 4:821-5 (Jan., '13).

Int. Studio 26:261-4 (Sept.,' 05 ).

Int. Studio 37: sup. 65-8 (May, '09).

Int. Studio 61: sup. 19-24 (Mar., '17).

\section{ROBERT HENRI}

Book: Hartmann.

Magazines: Amer. Mag. of Art 7:473-9 (Oct., '16).

Arts and Dec. 2:213-15 (Apr., '12).

Arts and Dec. 14:36 (Nov., '20).

Craftsman 27:459-69 (Feb., '15).

Ind. 64: pt. 2:1427-32 (June 25, '08).

Int. Studio 56:239-41 (Oct., '15).

*Touchstone 5:212-19 (June, '19).

WILLIAM H. HOLMES

Art and Prog. 6:11-14 (Nov., '14).

\section{CHARLES S. HOPKINSON}

Nation 92:369-70 (Apr., 13, '11).

\section{JOHN C. JOHANSEN}

Amer. Mag. of Art 10:290-295 (June, '19).

Int. Studio 26:264-67 (Sept., '05).

Int. Studio 42: sup. 3-6 (Nov., '10). 


\section{W. SERGEANT KENDALL}

Harper's 117:568-77 (Sept., '08).

\section{ROCKWELI KENT}

Book: Kent-Wilderness (G945-K418).

Magazines: Arts and Dec. 11:70-2 (June, '19).

Arts and Dec. 12:324-5 (Mar., '20).

Cur. Opinion 62:277-8 (Apr., '17).

Cur. Opinion 67:52-3 (July, '19).

Cur. Opinion 68:681-5 (May, '20).

Int. Studio 67: sup. 105-12 (June, '19).

Lit. Dig. 61:31-2 (May 31, '19).

\section{LEON KROLL}

Int. Studio 72:36-38 (Feb., '21).

\section{ERNEST LAWSON}

Amer. Mag. of Art 8:257-63 (May, '17).

Arts and Dec. 6:505-7 (Sept., '16).

Arts and Dec. 10:257-8 (Mar., '19).

Cur. Lit. 42:406-9 (Apr., '07).

Int. Studio 59: sup. 13-15 (July, '16).

Int. Studio 72: sup. 114-17 (Feb., '21).

\section{HAYLEY LEVER}

Int. Studio 52: sup. 92-3 (May, '14).

Int. Studio 70: sup. 67-70 (May, '20).

\section{JONAS LIE}

Arts and Dec. 12-91 (Dec., '19).

Arts and Dec. 15:221 (Aug., '21).

Bul. Pan. Amer. Union 38:679-83 (May, '14).

Century 99:67-74 (Nov., '19).

Country Life 36:55-7 (July, '19).

Craftsman 13:135-9 (Nov.,'07).

Cur. Lit. 52:222-4 (Feb.,'12).

Int. Studio 51: sup. 192-4 (Feb., '14). 


\section{PHILIP LITTLE}

Art and Prog. 5:19-21 (Nov., '13).

\section{WALTER McEWEN}

*Maitres Contemporains 46:8 (1908).

\section{GARI MELCHERS}

Books : Caffin-Story of American painting, Muther.

Magazines: Harper's 114:430-9 (Feb., '07).

Int. Studio 31: sup. 11-18 (Mar., '07).

Int. Studio 48: sup. 27-30 (Dec., '12).

World's Work 15:10092-105 (Apr., '08).

\section{WILLARD L. METCALF}

Century 77:155 (Nov., '08).

Country Life 38:36-7 (June, '20).

New Eng. Mag. n. s. 39:374-75 (Nov., '08).

\section{RICHARD E. MILLER}

Art and Prog. 6:39-40, 54-7 (Dec., '14). Int. Studio 43:263-73 (June, '11).

\section{JEROME MYERS}

Amer. Mag. of Art 8:481-5 (Oct., '17).

Art and Prog. 5:89-94 (Jan., '14).

Arts and Dec. 10:257, 259 (Mar., '19).

Craftsman 29:25-32 (Oct., '15).

Int. Studio 57: sup. 125-8 (Feb., '16).

*Touchstone 5:396-9 (Aug., '19).

\section{ROBERT FI. NISBET}

Int. Studio 49: sup. 11-14 (Mar., '13).

VIOLET OAKLEY

Books: Clement, Hartmann. 
Magazines: Arch. Rec. 22:455-68 (Dec., '07).

Century 70:265-8 (June, '05).

Century 81:734-8 (Mar., '11).

Lit Dig. 48:64-5 (Jan. 10, '14).

Scribner's 41:637-40 (May, '07).

Scribner's 62:125-8 (July, '17).

\section{WILLIAM M. PAXTON}

Int. Studio 39: sup. 46-8 (Dec., '09).

World Today 9:1210-14 (Nov., '05).

\section{VAN DEARING PERRINE}

Craftsman 12:486-96 (Aug., '07).

Cur. Lit. 41:407-10 (Oct.,'06).

*Touchstone 6:8-9 (Oct., '19).

\section{LILLA CABOT PERRY}

Book: Clement.

\section{CHARLES A. PLATT}

Book: *Monograph on the work of Charles A.

Platt, with introd. by Royal Cortissoz. 1913.

Magazines: Arch. Rec. 15:181-244 (Mar., '04).

Arch. Rec. 36:81-97 (Aug., '14).

Arch. Rec. 48:178-91 (Sept., '20).

\section{HENRY RANKIN POORE}

Int. Studio 37: sup. 20-4 (Mar., '09).

New Eng. Mag. n. s. 39:604-5 (Jan., '09).

\section{MAURICE B. PRENDERGAST}

World Today 19:716-19 (July, '10).

\section{HOVSEP PUSHMAN}

Art and Archaeol. 10:194-5 (Nov., '20).

Int. Studio 61: sup. 37-43 (Apr., '17).

Int. Studio 67: sup. 79-81 (May, '19). 


\section{EDWARD W. REDFIELD}

Arena 36:20-6 (July, '06).

Arts and Dec. 6:135 (Jan., '16).

Country Life 13:194-97 (Dec., '07).

Country Life 38:38-9 (June, '20).

Int. Studio 41: sup. 29-36 (Aug., '10).

\section{ROBERT REID}

Book: Hartmann.

Magazines: Arts and Dec. 2:13-15, 34 (Nov., '11).

Craftsman 7:699-712 (Mar., '05). Int. Studio 36: sup. 113-22 (Feb., '09).

\section{LOUIS RITMAN}

Int. Studio 67: sup. 62-4 (Apr., '19).

\section{WHLIAM RITSCHEL}

*Fine Arts J. 34:275-80 (June, '16). Int. Studio 28: sup. 50-51 (June, '06).

\section{JULIUS ROLSHOVEN}

Amer. Mag. of Art 11:359-60 (Aug., '20).

\section{ALBERT ROSENTHAL}

*Wilson's Photo. Mag. 49:310-15 (July, '12).

\section{CARL RUNGIUS}

Country Life 40:34-9 (Sept., '21).

\section{CHAUNCEY F. RYDER}

*Art Review 22-1:3 (Sept., '11).

JOHN SINGER SARGENT

Books: Brinton - Modern artists (W10-9B774 Reference), Caffin-American masters, 
Cortissoz - Art and common sense (W-C817), Cox - Artist and public (TV-C838), Hartmann, Isham, McSpadden, Muther, Sargent-Work of Sargent, with introd. by Mrs. Meynell (WPSa743 Reference), Van Dyke-American painting and its tradition (WPS3-V28). Magæzines: Amer. Mag. of Art 8:129-36 (Jan., '17).

Amer. Mag. of Art 12:401-7 (Dec., '21).

Art and Prog. 3:742-5 (Oct., '12). Arts and Dec. 7:194-7 (Feb., '17).

Forum 54:232-6 (Aug., '15). Int. Studio 10:3-21 (Mar., '00). Int. Studio 10:107-19 (Apr., '00). Scribner's 34:515-32 (Nov.,'03). World's Work 7:4099-118 (Nov., '03).

\section{W. ELMER SCHOFIELD}

Arts and Dec. 1:473-5 (Oct., '11). Int. Studio 48:280-6 (Feb., '13).

\section{EVERETT SHINN}

Book: Hartmann.

Magazines: Craftsman 21:378-85 (Jan., '12)

Ind. 88:398 (Dec. 4, '16).

Int. Studio 30:84-87 (Nov., '06).

Int. Studio 51: sup. 168-70 (Jan., '14).

\section{JOHN SLOAN}

Book: Caffin-Story of American painting.

Magazines: Craftsman 15:559-64 (Feb., '09).

Harper's Weekly 58:20-21 (Nov. 22, '13).

Int. Studio 58: sup. 25-8 (Mar., '16).

*Touchstone 4:362-70 (Feb., '19). 


\section{HENRY B. SNELL}

Book: Hartmann.

\section{EUGENE SPEICHER}

Int. Studio 46: sup. 19-20 (Mar., '12).

MAURICE STERNE

Cur. Opinion 59:425-7 (Dec., '15). Int. Studio 46: sup. 3-13 (Mar., '12). Int. Studio 61: sup. 53-6 (Apr., '17).

\section{GARDNER SYMONS}

*Fine Arts J. 34:557-65 (Nov., '16). Outlook 105:881-7 (Dec. 27, '13).

\section{AUGUSTUS VINCENT TACK}

Cur. Opinion 58:269-70 (Apr., '15). Int. Studio 54: sup. 3-10 (Nov., '14). Int. Studio 58: sup. 19-24 (Mar., '16).

\section{EDMUND C. TARBELL}

Book: Hartmann.

Magazines: Amer. Mag. of Art 9:217-28 (Apr., '18).

Arts and Dec. 2:129-31 (Feb., '12).

Harper's 117:65-74 (June, '08).

Int. Studio 32: sup. 75-87 (Sept., '07).

* Mentor 8:26-7 (Dec., '20).

World Today 11:1077-85 (Oct., '06).

\section{HELEN M. TURNER}

Art World 3:490 (Mar., '18).

\section{WALTER UFER}

*Fine Arts J. 34:221-6 (May, '16). 


\section{DOUGLAS VOLK}

Art World 3:282-3 (Jan., '18).

Century 68:654-5 (Aug., '04).

\section{ROBERT VONNOH}

Books: Hartmann, Muther.

Magazines: Art and Prog. 4:999-1002 (June, '13).

Arts and Dec. 2:381-3 (Sept., '12).

Int. Studio 54: sup. 48-52 (Dec., '14).

\section{HORATIO WALKER}

Books: Caffin-American masters, Hartmann.

Magazines: Arts and Dec. 1:63-5, 88 (Dec., '10).

Canad. Mag. 18:496-500 (Apr., '02).

Canad. Mag. 53:21-9 (May, '19).

Craftsman 14:138-45 (May, '08).

Harper's 117:947-56 (Nov., '08).

*Touchstone 5:482-5 (Sept., '19).

\section{FREDERICK J. WAUGH}

Arts and Dec. 1:111-13 (Jan., '11).

Century 100:641-8 (Sept., '20).

Int. Studio 51:273-8 (Feb., '14).

Int. Studio 60: sup. 50-2 (Dec., '16).

\section{WILLIAM WENDT}

Amer. Mag. of Art 7:232-35 (Apr., '16).

Art and Prog. 6:73-4 (Jan., '15).

*Brush and Pencil 6:257-63' (Sept., '00).

\section{GUY WIGGINS}

Arts and Dec. 10:310 (Apr., '19).

\section{IRVING R. WILES}

Art and Prog. 6:213-14 (May, '15). Arts and Dec. 1:402-4 (Aug.,' '11). Craftsman 18:347-53 (June, '10). 


\section{CHARLES H. WOODBURY}

Art and Prog. 4:761-6 (Nov., '12).

Int. Studio 42: sup. 71-78 (Feb., '11).

\section{S. J. WOOLF}

*Amer. Art News 17:2 (Feb.,1, '19).

\section{CHARLES MORRIS YOUNG}

Int. Studio 28:369-70 (June, '06).

\section{BOOKS}

\section{ART APPRECIATION}

Neuhaus, Eugen. Painters, pictures and the people. 1918. W-N394p.

Noyes, C. E. Gate of appreciation. 1907. $\mathrm{W}-\mathrm{N} 873 \mathrm{~g}$.

Sturgis, Russell. Appreciation of pictures. 1905. WP-St974ap.

Van Dyke, J. C. How to judge of a picture. 1889. WP-V289h.

Van Dyke, J. C. What is art? 1910. WPV289w.

\section{AMERICAN PAINTERS AND PAINTING}

CaIIn, C. H. American masters of painting. 1913. W10-9C112.

Caffin, C. H. Story of American painting. 1907. WP83-C11.

Clement, Mrs. C. E. Women in the fine arts. 1904. W10-9C59w.

Hartmann, Sadakichi. History of American art. 2v. 1902. W83-H255. 
Innes, Mary. Schools of painting. 1911. WPIn65s.

Isham, Samuel. History of American painting. 1907. WP-Is326h.

McSpadden, J. W. Famous painters of America. 1916. W10-9M246.

Muther, Richard. History of modern painting. v. 3. 1907. WP-91i984 Reference.

Who's Who in Art. (In American art annual, 1919.) WT-7Am Reference.

\section{COLLECTIVE ARTICLES}

The following magazine articles group several painters together, comparing them with American and European artists not exhibiting; some of them discuss certain later movements, tendencies and revolts against the established standards. They help to explain the great diversity of the work shown.

This transitional age in art: J. W. Alexander, Is our art distinctively American? E. H. Blashfield, The painting of today; E. L. Blumenschein, The painting of tomorrow; Walter Pach, The point of view of the "moderns"; Jay Hambridge and Gove Hambridge, The ancestry of Cubism. Century 87:825-75 (Apr., '14).

Special exhibition number of Arts and Decoration: Arthur B. Davies, Explanatory statement; G. P. DuBois, Spirit and chronology of the modern movement; John Quinn, Modern art from a layman's point of view; W. J. Glackens, American section - the national art; F. J. Gregg, Attitude of the Americans; W. M. Fisher, Sculpture at the exhibition; J. Davidson, The extremists-an interriew; M. Dodge, Speculations or post-impression- 
ism in prose. Arts and Decoration 3:149 (Mar., '13).

Some American portrait painters: Sargent, Eakins, Duveneck, Thayer, Brush, Henri, Alexander, Chase, Weir and Beaux. C. H. Caffin. Critic 44:31-45 (Jan., '04).

Some American marine painters: Carlsen, Dougherty, Waugh, Woodbury. Anna SeatonSchmidt. Art and Prog. 2:3-8 (Nov., '10).

Recent tendencies in marine painting: Birge Harrison. Scribner's 49:469-77 (Apr., '11).

Painters of the Palisades: Bailey Millard. Bookman 35:256-65 (May, '12).

Nature invites and art responds: The haunts of the painters of the Delaware Valley school. Harvey M. Watts. Arts and Dec. 15:153-55 (July, '21).

Contemporary American landscape painting. L. Mechlin. Int. Studio 39:3-14 (Nov., '09).

Some American figure painters. L. Mechlin. Int. Studio 39:185-194 (Jan., '10).

The Public Library has published several reference lists on the history of painting and on various phases of the fine arts which are still available. The assistants in the reference room are always glad to help students of art history or criticism to locate needed material. 

H3

\section{THE PUBLIC LIBRARY OF THE}

DISTRICT OF COLUMBIA

REFERENCE LIST No. 17

Contemporary American Painters.

\section{NATIOMAL COLECTION OF FINE ART:}

References to literature in books and periodicals on many of the exhibiters at the Ninth Exhibition of Contemporary American Oil Paintings, Corcoran Gallery of Art, December 16, 1923, to January 20, 1924, inclusive.

Issued with the cooperation of The Corcoran Gallery of Art

THE PUBLIC LIBRARY WASHINGTON, D. C.

December, 1923 



\section{CONTEMPORARY \\ AMERICAN PAINTERS}

Brief biographical information in regard to most of the painters exhibiting is given in "Who's Who in Art, 1921." When an artist is represented in that reference work or by slight mention in any of the other books included in the list, no specific note is made.

A valuable clipping file of material on American artists is maintained by the Corcoran Gallery of Art. Nearly all of the artists exhibiting are represented in this collection, which may be consulted by persons interested.

Under each exhibitor (so far as he has been written about), references are given to chapters in books, referred to only by their authors, listed below and to magazine articles. The items marked with a star (*) are not in the Public Library but may be found at the Library of Congress. All other books and periodicals referred to in this list may be found in the Public Library, Eighth and $\mathrm{K}$ Streets, N. W.

\section{BOOKS}

\section{AMERICAN PAINTERS AND PAINTING}

Caffin, C. H. American masters of painting. 1913. W10-9C112.

Caffin, C. H. Story of American painting. 1907. WP83-C11.

Clement, Mrs. C. E. Women in the fine arts. 1904. W10-9C59w. 
Cortissoz, Royal. American artists. 1923. W83-C817.

Hartmann, Sadakichi. History of American art. 2v. 1902. W83-H255.

Innes, Mary. Schools of painting. 1911. WP-In65s.

Isham, Samuel. History of American painting. 1907. WP-Is326h.

McSpadden, J. W. Famous painters of America. 1916. W10-9M246.

Muther, Richard. History of modern painting. v. 3. 1907. WP-9M984 Reference.

Who's Who in Art. (In American art annual, 1921.) W-7Am Reference.

\section{WAYMAN ADAMS}

Int. Studio 77:86-91 (May, '23).

\section{MARION BOYD ALLEN}

Art and Prog. 6:460-3 (Oct., '15).

\section{KARL ANDERSON}

Int. Studio 76:132-4 (Nov., '22).

\section{BURTIS BAKER}

Clipping file, Corcoran Gallery of Art.

GIFFORD BEAL

Int. Studio 77:236-42 (June, '23). *Touchstone 5:240-3 (June, '19).

\section{CECILIA BEAUX}

Books: Clement, Hartmann, Isham. Magazines: Amer. Mag. 96:61-3 (Oct., '23). Art World $3: 489-90$ (Mar., '18). Century 80:581-7 (Aug., '10). 
Ind. $103: 382-4$ (Sept. 25, '20). Int. Studio 8:215-22 (Oct., '99). Int. Studio 41 :sup.3-10 (July, '10).

Scribner's $22: 477-85$ (Oct., '97).

\section{GEORGE W. BELLOWS}

Book: Cortissoz.

Magazines: Art and Prog. 3:679-82 (Aug., '12).

Arts and Dec. 4:370-3 (Aug., '14).

Arts and Dec. 12:10 (N o v., '19).

Arts and Dec. $15: 158-9$ (July, '21).

Int. Studio $56: 242-4 \quad$ (O c t., '15).

Int. Studio 71 :sup.21-7 (Aug., '20).

New Repub. 23:220-1 (July 21, '20).

*Touchstone 1:269-75 (July, '17).

\section{FRANK W. BENSON}

Book: Isham.

Magazines: Amer. Mag. of Art 9:309-16 (June, '18).

Amer. Mag. of Art $12: 365-72$ (Nov., '21).

Art and Archaeol. $15: 92-5$ (Feb., '23).

Arts and Dec. 1:195-7 (Mar., '11).

Country Life 36:53-5 (Oct., '19). Harper's $119: 105-14$ (June, '09). 
Harper's 138:826 (May, '19).

Int. Studio 35 :sup.99-104 (Oct., 08).

Int. Studio 74:95-100 '21).

(Oct.,

\section{OSCAR E. BERNINGHAUS}

Country Life $33: 57-9$ (Apr., '18).

THERESA BERNSTEIN

Book: Bryant-American pictures and their painters (WP83-B844).

Magazine: Int. Studio 66: sup. 97-102 (Feb., '19).

\section{LOUIS BETTS}

Int. Studio $77: 523-5$ (Sept., '23).

\section{CHARLES BITTINGER}

Int. Studio 59: sup. 41-4 (Aug., '16).

\section{ERNEST L. BLUMENSCHEIN}

Outlook 134:504-7 (Aug. 1, '23).

\section{ADOLPHE BORIE}

*Touchstone 4:519-21 (Mar., '19).

\section{LESTER D. BORONDA}

Int. Studio 54: sup. 130-2 (Feb., '15).

\section{HUGH H. BRECKENRIDGE}

Amer. Mag. of Art $7: 85-6$ (Jan., '16). Int. Studio 37: sup. 34-6 (Mar., '09).

\section{PUTNAM BRINLEY}

*House and Garden $41: 34$ (Mar., '22). House Beautiful 44:361-3 (Dec., '18). 


\section{GEORGE DE FOREST BRUSH}

Books: Caffin-American masters; Story of American paintings, Cortissoz, Hartman, Isham.

Magazines: Art World 2:8-9 (Apr., '17).

Critic: $47: 134-5$ (Aug., '05). Int. Studio 34: sup. 47-56 (Apr., '08).

Int. Studio $76: 186-92$ (Dec., '22).

\section{BRYSON BURROUGHS}

Book: Isham.

Magazine: Arts and Dec. $12: 82-4$ (Dec., '19).

\section{MARGARET LESLEY BUSH-BROWN}

Art and Achaeol. $13: 189-90$ (Apr., '22).

\section{H. CALIGA}

New Eng. Mag. n. s. 32:110 (Mar., '05).

\section{ORLAND CAMPBELL}

Arts and Dec. 18:16-17 (Jan., '23). Cur. Opinion $73: 521$ (Oct., '22).

\section{DINES CARLSEN}

Amer. Mag. of Art 7:282 (May, '16).

\section{EMIL CARLSEN}

Book: Isham.

Magazines: Int. Studio 61: sup. 105-10 (June, '17).

Int. Studio $75: 300-8$ (July, '22). Scribner's 66:767-70 (Dec., '19). *Touchstone 7:110-16 (May, '20). 


\section{JOHN F. CARLSON}

Book: Bryant-American pictures and their painters (WP83-B844).

Magazine: *Fine Arts J. 35 :203-6 (Mar., '17).

\section{MARY CASSATT}

Books: Cary-Artists past and present (W109 C258), Clement, Cortissoz, Isham. Magazines: Arts and Dec. 3:265-7 (June, '13). Arts and Dec. 17:377 (Sept., '22). Craftsman 19:540-6 (Mar., '11). Cur. Opinion 62:426-7 (June, '17).

Int. Studio 27: sup. 1-7 (Nov., '05).

Print. Col. Q. 6:393-409 (Dec., '16).

Scribner's 19 :353-61 (Mar., '96). *World Today $21: 1659-62$ (Jan., '12).

\section{ALSON CLARK}

Clipping file, Corcoran Gallery of Art.

\section{RALPH E. CLARKSON}

Art and Archaeol. 12:199-201 (Nov., '21).

\section{JOHN E. COSTIGAN}

Clipping file, Corcoran Gallery of Art.

\section{E. IRVING COUSE}

Amer. Mag. of Art 11:400-5 (Sept., '20).

Century 99:369-76 (Jan., '20). Craftsman 18:619-25 (Sept., '10). Outlook 134:504-7 (Aug. 1, '23). 


\section{BRUCE CRANE}

*Brush and Pencil 11:1-10 (Oct., '02).

\section{CHARLES C. CURRAN}

Book: Hartmann.

Magazine: Critic 48:38-9 (Jan., '06).

\section{ELLIOTT DAINGERFIELD}

Book: Isham.

Magazines: Art World 3:370-2 (Feb., '18). *Mentor 5:1-11 (Oct. 15, '17).

\section{ARTHUR B. DAVIES}

Books: Cortissoz, Hartmann, Huneker- $\mathrm{Pa}-$ thos of distance (Y-H894p), Isham, Sherman - American painters of yesterday and today (W10-9Sh56). Magazines: Art and Archaeol. 4:169-77 (Sept., '16).

*Art in Amer. $6: 295-9$ (Oct., '18). Arts and Dec. 8:34, 36-7 (Nov., '17).

Cur. Opinion 64:203-5 (Mar., '18).

Forum 51:770-2 (May, '14). Int. Studio 72: sup. 126-30 (Feb., '21).

Int. Studio $75: 212-9$ (June,'22). Int. Studio $77: 254-5$ (June, '23). Lit. Dig. 56 :23-4 (Jan. 26, '18). New Repub. $13: 338$ (Jan. 19, '18).

*Touchstone 6:277-84 (Feb., '20).

\section{CHARLES H. DAVIS}

Book: Hartmann. 
Magazines: Int. Studio 75:176-83 (June, '22). New Eng. Mag. n. s. 27:423-37 (Dec., '02).

\section{THOMAS W. DEWING}

Books: Caffin-Story of American painting, Hartmann, Isham.

Magazines: Amer. Mag. of Art. 10:302-3 (June, '19).

Art World 3:188-9 (Dec., '17).

Critic 48:418-19 (May, '06).

Harper's $91: 165-70$ (July, '95).

Harper's 116:714-24 (Apr., '08).

\section{PAUL DOUGHERTY}

Arts and Dec. 8:251-4 (Apr., '18).

Int. Studio 30:180-2 (Dec., '06).

Int. Studio 36: sup. 3-4 (Nov., '08).

Int. Studio 73: sup. 54-8 (Apr., '21).

\section{LYDIA FIELD EMMET}

Book: Clement.

Magazine: Arts and Dec. 2:415-18 (Oct., '12).

\section{JOHN F. FOLINSBEE}

Int. Studio 73 : sup. 38 (Apr., '21).

Int. Studio $76: 420-6$ (Feb., '23).

\section{MARY FOOTE}

Book: Clement.

Magazine: Critic 37:131-8 (Aug., '00).

WILL HOWE FOOTE

*Arts, Aug.-Sept., '21. 
BEN FOSTER

*Fine Arts J. 34:176-80 (Apr., '16). Outlook 68:58-9 (May, '01).

\section{FREDERICK C. FRIESEKE}

Art and Prog. 3:747-9 (Oct., '12). Arts and Dec. $3: 13-15$ (Nov., '12). Int. Studio $53: 259-64$ (Oct., '14). Scribner's 58:643-6 (Nov., '15).

\section{DANIEL GARBER}

*Maitres Contemporains 59:10 (1912).

\section{WILLIAM J. GLACKENS}

Book: Isham.

Magazines: Amer. Mag. of Art $7: 261-3$ (May, '16).

Arts and Dec. 4:404-6 (Sept., '14).

Arts and Dec. 7:85 (Dec., '16).

Arts and Dec. 14:103 (Dec.,'20). Int. Studio 40: sup. 68 (May, '10).

*Touchstone 7:191-9 (June, '20).

WALTER GRIFFIN

Int. Studio 62: sup. 45-8 (Aug., '17).

\section{ALBERT L. GROLL}

Century 100:385-92 (July, '20). Craftsman 9:826-36 (Mar., '06). Int. Studio 28: sup. 78-82 (May, '06). Int. Studio $29: 270-1$ (Sept., '06). Int. Studio 64: sup. 105-10 (June, '18). Int. Studio $75: 496-501$ (Sept., '22). 


\section{PHILIP L. HALE}

Amer. Mag. of Art 7:259-60 (May, '16). *World Today 14:59-67 (Jan., '08).

\section{JOHN McLURE HAMILTON}

Book: Isham.

Clipping file, Corcoran Gallery of Art.

\section{WILBUR DEAN HAMILTON}

Clipping file, Corcoran Gallery of Art.

\section{GEORGE HARDING}

Amer. Mag. of Art 10:451-6 (Oct., '19). Int. Studio 62: sup. 16-18 (July, '17).

\section{CHILDE HASSAM}

Books: Caffin-Story of American painting, Cortissoz, Hartmann.

Magazines: Art and Prog. 2:137-9 (Mar.,

$$
\text { '11). }
$$

Int. Studio 45: sup. 29-30 (Dec., '11).

Int. Studio 57: sup. 83-6 (Jan., '16).

Int. Studio $77: 2-7$ (April, '23).

Nation 101:698-9 (Dec. 9, '15). *Touchstone $5: 272-80$ (July, '19).

\section{CHARLES W. HAWTHORNE}

Art and Prog. $4: 821-5$ (Jan., '13).

Int. Studio 26:261-4 (Sept., '05).

Int. Studio 37: sup. 65-8 (May, '09).

Int. Studio 61: sup. 19-24 (Mar., '17).

\section{ROBERT HENRI}

Books: Cortissoz, Hartmann, *Yarrow, ed.Robert Henri, his life and works, 1921. 
Magazines: Amer. Mag. of Art 7:473-9 (Oct., '16).

Arts and Dec. 2:213-15 (Apr., '12).

Arts and Dec. 14:36 (Nov., '20). Arts and Dec. 20:26 (Dec., '23). Craftsman 27:459-69 (Feb., '15). Cur. Opinion 71:793-6 (Dec., '21).

Ind. 64 : pt. $2: 1427-32$ (June 25, '08).

Int. Studio $56: 239-41$ (Oct., '15). Survey 50: 270-82 (June 1,'23). *Touchstone 5:212-19 (June, '19).

\section{LOUISE LYONS HEUSTIS}

Book: Clement.

Magazine: Int. Studio 42: sup. 15-17 (Nov., '10).

EUGENE HIGGINS

*Touchstone 5:360-9 (Aug., '19).

WILLIAM H. HOLMES

Art and Prog. 6:11-14 (Nov., '14).

JAMES R. HOPKINS

*Mentor 8:32-3 (Dec., '20).

\section{CHARLES HOPKINSON}

Nation 92:369-70 (Apr. 13, '11).

\section{EDWARD HOPPER}

*Shadowland, Oct., '22, pp. 22-3.

\section{ERIC HUDSON}

Int. Studio 75 :2-6 (Mar., '22). 


\section{FRANK T. HUTCHENS}

Int. Studio 47:160-1 (Aug., '12).

\section{JOHN C. JOHANSEN}

Amer. Mag. of Art 10:290-5 (June, '19). Int. Studio $26: 264-7$ (Sept., '05). Int. Studio 42: sup. 3-6 (Nov., '10).

\section{ROCKWELL KENT}

Book: Kent-Wilderness, 1920 (G945-K418). Magazines: Arts and Dec. 11:70-2 (June, '19).

Arts and Dec. 12:324-5 (Mar., '20).

Cur. Opinion 62:277-8 (Apr., '17).

Cur. Opinion $67: 52-3$ (July, '19). Cur. Opinion 68:681-5 (May, '20).

Int. Studio 67: sup. 105-12 (June, '19).

Lit. Dig. 61:31-2 (May 31, '19).

\section{LEON KROLL}

Int. Studio 72:36-8 (Feb., '21).

\section{ERNEST LAWSON}

Amer. Mag. of Art 8:257-63 (May, '17). Arts and Dec. 6 :505-7 (Sept., '16). Arts and Dec. 10:257-8 (Mar., '19). Cur. Lit. 42:406-9 (Apr., '07). Int. Studio 59: sup. 13-15 (July, '16). Int. Studio 72: sup. 114-17 (Feb., '21).

\section{HAYLEY LEVER}

Int. Studio 52 : sup. 92-3 (May, '14). Int. Studio 70: sup. 67-70 (May, '20). 


\section{JONAS LIE}

Arts and Dec. 12:91 (Dec., '19).

Arts and Dec. 15:221 (Aug., '21).

Century $99: 67-74$ (Nov., '19).

Country Life 36:55-7 (July, '19).

Craftsman 13:135-9 (Nov., '07).

Cur. Lit. $52: 222-4$ (Feb., '12).

Int. Studio 51 sup. 192-4 (Feb., '14).

Pan Amer. Union Bul. 38:679-83 (May, '14).

\section{CORWIN KNAPP LINSON}

Century 69:548-56 (Feb., '05).

\section{WALTER McEWEN}

Book: Isham.

Magazine: *Maitres Contemporains $46: 8$ (1908)

\section{GARI MELCHERS}

Books: Caffin-Story of American painting, Isham, Muther.

Magazines: Amer. Mag. of Art 13:117-21 (Apr., '22).

Harper's 114:430-9 (Feb., '07). Int. Studio 31 sup. 11-18 (Mar., '07).

Int. Studio 48: sup. 27-30 (Dec., '12).

World's Work 15:10092-105 (Apr., '08).

\section{RICHARD S. MERYMAN}

Clipping file, Corcoran Gallery of Art.

\section{WILLARD L. METCALF}

Books: Cortissoz, Isham. 
Magazines: Century 77:155 (Nov., '08). Country Life 38:36-7 (June'20). New Eng. Mag. n. s. $39: 374-5$ (Nov., '08).

\section{WILLIAM MEYEROWITZ}

Clipping file, Corcoran Gallery of Art.

\section{KENNETH HAYES MILLER}

New Rep. $27: 244-5$ (July 27, '21).

\section{RICHARD E. MILLER}

Art and Prog. 6:39-40, 54-7 (Dec., '14). Int. Studio $43: 263-73$ (June '11).

\section{F. LUIS MORA}

Lit. Dig. 64:40-1 (Mar., 27, '20).

\section{H. DUDLEY MURPHY}

*Sketch Book 6:303-6 (Nov., '07).

\section{JEROME MYERS}

Book: Isham.

Magazines: Amer. Mag. of Art 8:481-5 (Oct., '17).

Art and Prog. 5:89-94 (Jan., '14).

Arts and Dec. $10: 257,259$ (Mar., '19).

Craftsman 29:25-32 (Oct., '15). Int. Studio 57 sup. 125-8 (Feb., '16).

Survey 51:33-9 (Oct., 1, '23). *Touchstone 5:396-9 (Aug., '19).

\section{JOHN NOBLE}

Int. studio 77:457-65 (Sept., '23). 


\section{VIOLET OAKLEY}

Books: Clement, Hartmann.

Magazines: Arch. Rec. 22:455-68 (Dec., '07). Arts and Dec. 12:19 (Nov., '19). Century 70:265-8 (June, '05). Century 81:734-8 (Mar., '11). Lit. Dig. 48:64-5 (Jan. 10, '14). Scribner's 41:637-40 (May, '07). Scribner's $62: 125-8$ (July, '17).

\section{GEORGE OBERTEUFFER}

Int. Studio 43:275-80 (June, '11).

WILLIAM M. PAXTON

Int. Studio 39: sup. 46-8 (Dec., '09).

*World Today 9:1210-14 (Nov., '05).

\section{VAN D. PERRINE}

Craftsman 12:486-96 (Aug., '07).

Cur. Lit. 41:407-10 (Oct., '06).

*Touchstone 6:8-9 (Oct., '19).

BERT G. PHILLIPS

Outlook 134:504-7 (Aug. 1, '23).

CHARLES A. PLATT

Book: *Monograph on the work of Charles A. Platt, with introd. by Royal Cortissoz. 1913.

Magazines: Arch. Rec. 15 :181-244 (Mar., '04).

Arch. Rec. 36:81-97 (Aug., '14). Arch. Rec. 48:178-91 (Sept., '20). Arch. Rec. 51:16-32 (Jan., '22).

\section{EDWARD H. POTTHAST}

Clipping file, Corcoran Gallery of Art 


\section{MAURICE B. PRENDERGAST}

Book: Isham.

Magazine: *World Today 19:716-19 (July, '10).

\section{EDWARD W. REDFIELD}

Arena 36:20-6 (July, '06).

Arts and Dec. 6:135 (Jan., '16).

Country Life 13:194-7 (Dec., '07).

Country Life 38:38-9 (June, '20).

Int. Studio 41: sup. 29-36 (Aug., '10).

Int. Studio 75:402-10 (Aug., '22).

\section{WILLIAM RITSCHEL}

*Fine Arts J. 34:275-80 (June, '16).

Int. Studio 28: sup. 50-1 (June, '06).

\section{ALBERT ROSENTHAL}

*Wilson's Photo. Mag. 49:310-15 (July, '12).

\section{ORLANDO ROULAND}

Century 72:856-8, 961 (Oct., '06). Int. Studio 31: sup. 122-30 (June, '07).

\section{CARL RUNGIUS}

Country Life 40:34-9 (Sept., '21)

\section{JOHN SINGER SARGENT}

Books: Brinton-Modern artists (W10-9B774 Reference), Caffin-American masters, Cortissoz-Art and common sense (W-C817), Cox-Artist and public (W-C838), Hartmann, Isham, James-Picture and text (Y-J2294p), McSpadden, Muther, Sargent-Work of Sargent, with introd. by Mrs. Meynell (WP- 
Sa743 Reference), Van DykeAmerican painting and its tradition (WP83-V28).

Magazines: Amer. Mag. of Art 8:129-36 (Jan., '17),

Amer. Mag. of Art 12:401-7

(Dec., '21).

Amer. Mag. of Art $14: 3-6$ (Jan., '23).

Art and Prog. $3: 742-5$ (Oct., '12).

Arts and Deic. 7:194-7 (Feb., '17).

Dial 75 :443-6 (Nov., '23).

Forum 54 :232-6 (Aug.,' 15).

Int. Studio 10:3-21 (Mar., '00). Int. Studio 10:107-19 (Apr., '00).

Lit. Dig. 71 :26-7 (Nov., 26, '21). Lit. Dig. $73: 30-1$ (June 10,'22). Scribner's 71:379-84 (Mar.,' 22).

Book: Isham.

\section{W. ELMER SCHOFIELD}

Magazines: Arts and Dec. 1:473-5 (Oct., '11).

Int. Studio 48:280-6 (Feb., '13).

LEOPOLD SEYFFERT

Book: Bryant-American pictures and their painters (WP83-B844).

\section{HENRY B. SNELL}

Books: Hartmann, Isham.

ROBERT SPENCER

Int. Studio 76:485-91 (Mar., '23). 


\section{MAURICE STERNE}

Cur. Opinion 59:425-7 (Dec., '15). Int. Studio 46 sup. 3-13 (Mar., '12). Int. Studio 61: sup. 53-6 (Apr., '17). *Theatre Arts 7:15-20 (Jan.,' '23).

GARDNER SYMONS

*Fine Arts J. 34:557-65 (Nov., '16). Outlook 105:881-7 (Dec. 27, '13).

\section{AUGUSTUS VINCENT TACK}

Cur. Opinion 58:269-70 (Apr., '15). Int. Studio 54: sup. 3-10 (Nov., '14). Int. Studio 58: sup. 19-24 (Mar., '16).

\section{HENRY O. TANNER}

Int. Studio 50: sup. 11-15 (July, '13).

\section{EDMUND C. TARBELL}

Book: Hartmann.

Magazines: Amer. Mag. of Art 9:217-28 (Apr., '18).

Arts and Dec. 2:129-31 (Feb., '12).

Harper's 117:65-74 (June, '08). Int. Studio 32 : sup. 71-87 (Sept., '07).

*Mentor 8:26-7 (Dec., '20). *World Today 11:1077-85 (Oct., '06).

\section{GIOVANNI B. TROCCOLI}

House Beautiful 43:13-15 (Dec., '17).

\section{ALLEN TUCKER}

Int. Studio 52: sup. 19-20 (Mar., '14). 


\section{HELEN M. TURNER}

\section{Art World 3:490 (Mar., '18).}

\section{WALTER UFER}

Amer. Mag of Art 13:507-14 (Dec., '22). Art and Archaeol. 16:66-74 (Aug., '23). *Fine Arts J. 34:221-6 (May, '16). Int. Studio 77:293-9 (July, '23).

\section{DOUGLAS VOLK}

Amer. Mag. of Art 14:14-15 (Jan., '23). Art World $3: 282-3$ (Jan., '18). Century 68:654-5 (Aug.,' '04).

\section{HORATIO WALKER}

Books: Caffin-American masters, Hartmann, Isham.

Magazines: Arts and Dec. 1:63-5, 88 (Dec.,

$$
\text { '10). }
$$

Canad. Mag. 18:496-500 (Apr., '02).

Canad. Mag. 53 :21-9 (May, '19). Craftsman 14:138-45 (May, '08).

Harper's 117:947-56 (Nov., '08). Int. Studio 77:358-63 (Aug., '23).

*Touchstone 5:482-5 (Sept., 19).

\section{MARTHA WALTER}

Int. Studio 52: sup. 42-5 (Apr., '14).

\section{FREDERICK J. WAUGH}

Amer. Mag. of Art 13:15-18 (Jan., '22). Arts and Dec. 1:111-13 (Jan., '11). Century 100: 641-8 (Sept., '20). Int. Studio 51:273-8 (Feb., '14). 
Int. Studio 60: sup. 50-2 (Dec., '16).

Int. Studio 74 : sup. 125-35 (Dec., '21).

\section{GUY WIGGINS}

Arts and Dec. 10:310 (Apr., '19).

\section{IRVING R. WILES}

Art and Prog. 6:213-14 (May, '15). Arts and Dec. 1:402-4 (Aug., '11). Craftsman 18:347-53 (June, '10). Harper's $109: 802-3$ (Oct., '04). Int. Studio 77:256-62 (June, '22). Rev. of Rev. $34: 40-2$ (July, '06).

\section{CHARLES H. WOODBURY}

Art and Prog. 4:761-6 (Nov., '12). Int. Studio 42: sup. 71-8 (Feb., '11).

\section{CHARLES MORRIS YOUNG}

Int. Studio 28:369-70 (June, '06).

The Public Library has published several reference lists on the history of painting and on various phases of the fine arts which are still available. The assistants in the reference room are always glad to help students of art history or criticism to locate needed material. 

226 THE PUBLIC LIBRARY OF THE DISTRICT OF COLUMBIA

REFERENCE LIST No. 19

Contemporary American Painters

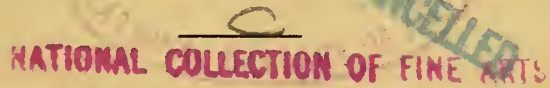

References to literature in books and periodicals on many of the exhibitors at the Tenth Exhibition of Contemporary American Oil Paintings, Corcoran Gallery of Art, April 4 to May 16, 1926, inclusive.

Issued with the cooperation of The Corcoran Gallery of Art

THE PUBLIC LIBRARY WASHINGTON, D. C.

April, 1926 


\section{CONTEMPORARY \\ AMERICAN PAINTERS}

Brief biographical information in regard to most of the painters exhibiting is given in "Who's Who in Art, 1925." When an artist is represented in that reference work or by slight mention in any of the other books included in the list, no specific note is made.

A valuable clipping file of material on American artists is maintained by the Corcoran Gallery of Art. Nearly all of the artists exhibiting are represented in this collection, which may be consulted by persons interested.

A Biographical Index of American Painters, Etchers, Engravers and Illustrators, available in manuscript only, was prepared in 1925 by Mr. Ralph C. Smith, Division of Graphic Arts, U. S. National Museum. Mr. Smith has also contributed references for inclusion in this list.

Under each exhibitor (so far as he has been written about), references are given to chapters in books, referred to only by their authors, listed below and to magazine articles. A dagger $(\dagger)$ in front of a name indicates that material about the artist has been found only in the clipping file of the Corcoran Gallery of Art. The items marked with a star (*) are not in the Public Library but may be found at the Library of Congress. All other books and periodicals referred to in this list may be found in the Public Library, Eighth and K Streets, N. W. 
Caffin, C. H. Story of American painting. 1907. WP83-C11.

Clement, Mrs. C. E. Women in the fine arts. 1904. W10-9 C59w.

Cortissoz, Royal. American artists. 1923. W83-C817.

Hartmann, Sadakichi. History of American art. 2v. 1902. W83-H255.

Innes, Mary. Schools of painting. 1911. WP-In65s.

Isham, Samuel. History of American painting. 1907. WP-Is326h.

MicSpadden, J. W. Famous painters of America. 1916. W10-9M246.

Muther, Richard. History of modern painting. v. 3. 1907. WP-9M984 Reference.

Who's Who in Art. (In American art annual, 1925). W-7Am Reference.

\section{ARTISTS IN EXHIBITION}

Wayman Adams. Int. Studio 77:86-91, May, 1923.

Karl Anderson. Int. Studio $76: 132-4$, Nov., 1922.

† Burtis Baker.

Gifford Beal. Int. Studio 44: sup. 20-30, Aug., 1911. Int. Studio 77:236-42, June, 1923. *Touchstone $5: 240-3$, June, 1919.

Cecilia Beaux. Books: Clement, Hartmann, Isham. Magazines: Amer. Mag. 96:61-3, Oct., 1923. Art World 3:489-90, Mar., 1918. Century 80:581-7, Aug., 1910. Ind. 103:382-4, Sept. 25, 1920. Int. Studio 8:215-22, Oct., 1899. Int. Studio 41: sup. 3-10, July, 1910. * Mentor 12:30-44, Oct., 1924. Scribner's 22: $477-85$, Oct., 1897 . Woman Cit. n. s. $9: 12-13$, Feb. 7, 1925 . 
Gerrit A. Beneker. Ind. $113: 43-4$, July 19 , 1924. Int. Studio 73: sup. 63-6, Apr., 1921. Lit. Dig. 85:28-9, June 20, 1925 . *Mentor $13: 43-50$, Sept., 1925 . Scribner's $74: 290-300$, Sept., 1923.

Frank W. Benson. Amer. Mag. of Art $9: 309-16$, June, 1918. Amer. Mag. of Art 12: 365-72, Nov., 1921. Art and Archaeol. 15: 92-5, Feb., 1923. Arts and Dec. 1:195-7, Mar., 1911. Country Life 36:53-5, Oct., 1919. Harper's 119:105-14, June, 1909. Ind. 113:4814, Dec. 6, 1924. Int. Studio 35: sup. 99-104, Oct., 1908. Int. Studio 74:95-100, Oct., 1921.

Theresa F. Bernstein. Int. Studio 66: sup. 97-102, Feb., 1919.

Ernest L. Blumenschein. Outlook 134: 504-7, Aug. 1, 1923.

Adolphe Borie. *Touchstone 4:519-21, Mar. 1919.

†Frederick A. Bosley.

† Jessie Arms Botke.

Hugh H. Breckenridge. Amer. Mag. of Art $7: 85-6$, Jan., 1916. Int. Studio 37: sup. 34-6, Mar., 1909.

†R. Sloan Bredin.

D. Putnam Brinley. *House and Garden $41: 34$, Mar., 1922. House Beautiful $44: 361-3$, Dec., 1918. Int. Studio 83:53-7, Jan., 1926.

Roy Brown. Int. Studio 83:78-82, Jan., 1926.

George Elmer Browne. Int. Studio 36:28692, Feb., 1909.

Margaret Fitzhugh Browne. Amer. Mag. of Art 16:408-11, Aug., 1925.

George De Forest Brush. Books: CaffinAmerican masters of painting (W10-9C112), Story of American painting, Cortissoz, Hartman, Isham. Magazines: *Art in Amer. 11: 200-7, June 1923. Art World 2:8-9, Apr., 1917. Critic $47: 134-5$, Aug., 1905. Int. Studio 34 : sup. 47-56, Apr., 1908. Int. Studio $76: 186$ 92, Dec., 1922. 
†Cameron Burnside.

Margaret Lesley Bush-Brown. Art and Archaeol. 13:189-90, Apr., 1922.

I. H. Caliga. New Eng. Mag. n. s. 32:110, Mar., 1905.

\section{†Arthur B. Caries.}

Dines Carlsen. Amer. Mag. of Art 7:282, May, 1916.

Emil Carlsen. Book: Isham. Magazines: Int. Studio 61: sup. 105-10, June, 1917. Int. Studio $75: 300-8$, July, 1922. Scribner's 66: 767-70, Dec., 1919. *Touchstone 7:110-16, Aay, 1920.

John F. Carlson. *Fine Arts J. $35: 203-6$; Mar., 1917.

\section{†William L. Carrigan.}

John Carroll. *Colour $13: 33$, Sept., 1920.

Ettore Caser. Amer. Mag. of Art 15:145-9, Mar., 1924.

Mary Cassatt. Books: Cary-Artists past and present (W10-9C258), Clement, Cortissoz, Isham. Magazines: Arts and Dec. 3:2657, June, 1913. Arts and Dec. $17: 377$, Sept., 1922. Craftsman 19:540-6, Mar., 1911. Cur. Opinion 62:426-7, June, 1917. Int. Studio 27: sup. 1-7, Nov., 1905. Print. Col. Q. 6:393-409, Dec., 1916. Scribner's 19:353-61, Mar., 1896. *World Today 21:1659-62, Jan., 1912.

†Charles S. Chapman.

John E. Costigan. Int. Studio 80:426-32, Mar., 1925.

E. Irving Couse. Amer. Mag. of Art 11: 400-5, Sept., 1920. Century 99:369-76, Jan., 1920. Craftsman 18:619-25, Sept., 1910. Outlook $134: 504-7$, Aug. 1, 1923.

Bruce Crane. * Brush and Pencil 11:1-10, Oct., 1902.

†Catharine C. Critcher. 
Arthur B. Davies. Books: Cortissoz, Hartmann, Huneker-Pathos of distance ( $\mathrm{Y}$ H894p), Isham, *Phillips Memorial Art Gallery-Arthur B. Davies, 1924, ShermanAmerican painters of yesterday and today (W10-9Sh56). Magazines: Art and Archaeol. $4: 169-77$, Sept., 1916. *Art in Amer. 6:295-9, Oct., 1918. Arts and Dec. 8:34, 36-7, Nov., 1917. Cur. Opinion 64:203-5, Mar., 1918. Forum 51:770-2, May, 1914 . Int. Studio 72: sup. 126-30, Feb., 1921. Int. Studio $75: 212-9$, June, 1922. Int. Studio $77: 254-5$, June, 1923. Lit. Dig. $56: 23-4$, Jan. 26, 1918. New Repub. $13: 338$, Jan. 19, 1918 . *Print Connoisseur $3: 194-213$, July, 1923. *Touchstone $6: 277-84$, Feb., 1920.

Charles H. Davis. Book: Hartmann. Magazines: Int. Studio $75: 176-83$, June, 1922 . New Eng. Mag. n. s. 27:423-37, Dec., 1902.

Thomas W. Dewing. Books: Cafîn, Cortissoz, Hartmann, Isham. Magazines: Amer. Mag. of Art 10:302-3, June, 1919. Art and Prog. 5:155-61, Mar., 1914. *Art in Amer. $10: 225-29$, Aug., 1922. Art World 3:188-9, Dec., 1917. Critic 48:418-19, May, 1906. Harper's 91:165-70, July, 1895. Harper's 116:714-24, Apr., 1908.

Paul Dougherty. *Art Review 23:1-3, Nov., 1911. Arts and Dec. 8:251-4, Apr., 1918. Int. Studio 30:180-2, Dec., 1906. Int. Studio 36: sup. 3-4, Nov., 1908. Int. Studio 73: sup. 54-8, Apr., 1921.

Frank V. Dumond. *Touchstone 5:262-4, June, 1919.

W. Herbert Dunton. Amer. Mag. of Art $15: 501-8$, Oct., 1924.

†Charles Ebert.

Truman E. Fassett. *Colour 18:85, May, June, 1923. 
Nicolai Fechin. *Amer. Art News 10:1, Dec. $30,1311$.

\section{Gertrude Fiske.}

John Folinsbee. Int. Studio 73: sup. 38, A pr., 1921. Int. Studio 76:420-6, Feb., 1923.

Will Foster. Scribner's 65:449-55, Apr., 1919.

Frederick C. Frieseke. Art and Prog. 3:747-9, Oct., 1912. Arts and Dec. 3:13-15, Nov., 1912. Int. Studio 53:259-64, Oct., 1914. Scribner's 58:643-6, Nov., 1915.

Maurice Fromkes. Art and Archaeol. 19: 162-74, Apr., 1925. Arts and Dec. 22:34-35, Dec., 1924. Century 77:958, Apr., 1909. Country Life $47: 86-90$, Nov., 1924 . Int. Studio $80: 396-403$, Feb., 1925. *Touchstone, 3:302-8, July, 1918.

Daniel Garber. *Maitres Contemporains $59: 10,1912$.

Leon Gaspard. Int Studio 78:16-21, Oct. 1923. Scribner's 59:281-8, Mar., 1916.

Lillian Genth. *Fine Arts J. 37:37-9, June, 1919. Int. Studio 50: sup. 55-61, Oct., 1913.

William J. Glackens. Book: Isham. Magazines: Amer. Mag. of Art 7:261-3, May, 1916. *Arts 3:246-60, Apr., 1923. Arts and Dec. 4:404-6, Sept., 1914. Arts and Dec. 7:85, Dec., 1916. Arts and Dec. 14:103, Dec., 1920. Int. Studio 40: sup. 68, May, 1910 . *Touchstone 7:191-9, June, 1920.

†Walter Goltz.

$\dagger$ A. H. Gorson.

†Edmund W. Greacen.

Walter Griffin. Int. Studio 62: sup. 45-8, Aug., 1917. 
Albert L. Groll. *Brush and Pencil 11:3641, 1902. * Brush and Pencil 18:43-51, Aug., 1906. Century 100:385-92, July, 1920. Craftsman 9:826-36, Mar., 1906. Int. Studio 28: sup. 78-82, May, 1906. Int. Studio 29:2701, Sept., 1906. Int. Studio 64: sup. 105-10, June, 1918. Int. Studio 75:496-501, Sept., 1922.

Oliver Dennett Grover. Int. Studio 26:267, Sept., 1905.

†Johanna K. W. Hailman.

Ellen Day Hale. Book: Clement, Women in the fine arts, (W10-9C59W). Clipping file, Corcoran Gallery of Art.

\section{†Lilian Westcott Hale.}

Philip L. Hale. Amer. Mag. of Art 7:259-60, May, 1916. *World Today 14:59-67, Jan., 1908.

Samuel Halpert. Int. Studio 75:144-50, Apr., 1922.

\section{†Wilbur Dean Hamilton.}

George Harding. Amer. Mag. of Art 10: 451-6, Oct. 1919. Int. Studio 62: sup. 16-18, July, 1917.

Childe Hassam. Books: Caffin, Cortissoz, Hartmann, McSpadden. Magazines: Art and Prog. 2:137-9, Mar., 1911. Int. Studio 45: sup. 29-30, Dec., 1911. Int. Studio 57: sup. 83-6, Jan., 1916. Int. Studio 77:2-7, Apr., 1923. Nation 101: 698-9, Dec. 9, 1915. *Touchstone 5:272-80, July, 1919.

Charles W. Hawthorne. Art and Prog. 4: 821-5, Jan., 1913. Int. Studio 26 :261-4, Sept., 1905. Int. Studio 37: sup. 65-8, May, 1909. Int. Studio 61: sup. 19-24, Mar., 1917.

Mary Brewster Hazelton. Book: Clement. 
Robert Henri. Books: Cortissoz, Hartmann, Henri-Art spirit (W-H397), Robert Henri, comp. by Pousette-Dart, (W10-H397), *Yarrow, ed.- Robert Henri, his life and works, 1921. Magazines: Amer. Mag. of Art $7: 473-9$, Oct., 1916. Arts and Dec. $2: 213-15$, Apr., 1912. Arts and Dec. 14:36, Nov., 1920. Arts and Dec. $20: 26$, Dec., 1923. Craftsman 27: 459-69, Feb., 1915. Cur. Opinion 71:793-6, Dec., 1921. Ind. 64: pt. 2:1427-32, June 25, 1908. Int. Studio 56:239-41, Oct., 1915. Lit. Digest $80: 27-8$, Jan. 5, 1924 . Survey 50: 27982, June 1, 1923. *Touchstone 5:212-19, June, 1919.

Eugene Higgins. Arts and Dec. 5:134-5, 164-5, Feb., 1915. Craftsman 12:134-46, May, 1907. Cur. Lit. 43:401-4, Oct., 1907. *Fine Arts J., Oct., $1907 . \quad$ *Touchstone 5 :360-9, Aug., 1919.

William H. Holmes. Art and Prog. 6:11-14, Nov., 1914.

James R. Hopkins. *Mentor 8:32-3, Dec., 1920.

Charles Hopkinson. Nation 92:369-70, Apr. $13,1911$.

Eric Hudson. Int. Studio $75: 2-6$, Mar., 1922.

$\dagger$ Wilson Irvine.

†Alexander R. James.

John C. Johansen. Amer. Mag. of Art 10: 290-5, June, 1919. Int. Studio 26:264-7, Sept., 1905. Int. Studio 42 : sup. 3-6, Nov., 1910.

†William Kaula. 
Rockwell Kent. Books: Kent-Voyaging southward from the Strait of Magellan, 1924 (G995T-K4), Wilderness, 1920 (G945-K418). Magazines: Amer. Mag. $99: 18-21$, Mar., 1925. Arts and Dec. 11:70-2, June, 1919. Arts and Dec. 12:324-5, Mar., 1920. Cur. Opinion 62: 277-8, Apr., 1917. Cur. Opinion 67:52-3, July, 1919. Cur. Opinion 68:681-5, May, 1920. Int. Studio 67: sup. 105-12, June, 1919. Int. Studio $79: 272-6$, July, 1924. Lit. Dig. 61:31-2, May 31, 1919.

Carl R. Krafft. *Fine Arts J. 34:465-72, Oct., 1916.

Leon Kroll. Int. Studio 72:36-8, Feb., 1921.

Louis Kronberg. Int. Studio 28:74-5, Mar., 1906. New Eng. Mag. n. s., 38:498-9, June, 1908.

Max Kuehne. Arts and Dec. 15:31, May, 1921. Int. Studio 68: sup. 37-40, Aug., 1919. Int. Studio 79:58-62, Apr., 1924.

William Lathrop. Int. Studio 78:132-8, Nov., 1923.

Ernest Lawson. Amer. Mag. of Art 8:25763, May, 1917. *Art in Amer. 8:32-39, Dec., 1919. Arts and Dec. 6:505-7, Sept., 1916. Arts and Dec. 10:257-8, Mar., 1919. Cur. Lit. 42:406-9, Apr., 1907. Int. Studio 59: sup. 13-15, July, 1916. Int. Studio 72: sup. 114-17, Feb., 1921. Int. Studio 78: 366-70, Feb., 1924.

Hayley Lever. Int. Studio 52: sup. 92-3, May, 1914. Int. Studio 70: sup. 67-70, May, 1920.

Jonas Lie. Amer. Mag. of Art 16:58-66, Feb., 1925. Arts and Dec. 12:91, Dec., 1919. Arts and Dec. 15:221, Aug., 1921. Country Life 36:55-7, July, 1919. Craftsman 13:1359, Nov., 1907. Cur. Lit. $52: 222-4$, Feb., 1912. Int. Studio 51: sup. 192-4, Feb., 1914. Int. Studio 82:102-7, Nov., 1925. Pan Amer. Union Bul. 38:679-83, May, 1914. 
Walter McEwen. Book: Isham. Magazine: * Maitres Contemporains 46:8, 1908.

M. Jean MacLane (Mrs. John C. Johansen). Art and Prog. 3:612, June, 1912. Arts and Dec. $3: 299-301$, July, 1913. Harper's 118: 291-3, Jan., 1909.

Ernest L. Major. Century $42: 229,318$, June, 1891.

Gari Melchers. Books: Caffin, Isham, Muther. Magazines: Amer. Mag. of Art 13: 117-21, Apr., 1922. Amer. Mag. of Art 15: 79-85, Feb., 1924. Arts and Dec. 24:40-1, Nov., 1925. Harper's 114:430-9, Feb., 1907. Int. Studio 31: sup. 11-18, Mar., 1907. Int. Studio 48: sup. 27-30, Dec., 1912. World's Work 15: 10092-105, Apr., 1908.

$\dagger$ Richard S. Meryman.

\section{$\doteqdot$ William Meyerowitz}

Kenneth Hayes Miller. New Repub. 27: 244-5, July 27, 1921.

Richard E. Miller. Art and Prog. 6:39-40, 54-7, Dec., 1914. Int. Studio 43:263-73, June, 1911.

F. Luis Mora. Lit. Dig. 64:40-1, Mar. 27, 1220.

\section{†Sarah Munroe}

H. Dudley Murphy. *Sketch Book 6:303-6, Nov., 1907.

Jerome Myers. Book: Isham. Niagazines: Amer. Mag. of Art 8:481-5, Oct., 1917. Art and Prog. 5:89-94, Jan., 1914. Arts and Dec. $10: 257$, 259, Mar., 1919. Craftsman $29: 25-$ 32, Oct., 1915. Int. Studio 57: sup. 125-8, Feb., 1916. Survey 51:33-9, Oct., 1, 1923. Touchstone 5:396-9, Aug., 1919. 
Hobart Nichols. Washington Star. Apr. 20, 1924, pt.3, p.5.

John Noble. Int. Studio 77:457-65, Sept., 1923.

George L. Noyes. Int. Studio 52: sup. 48-9, Apr., 1914.

Ivan G. Olinsky. Lit. Dig. $83: 29$, Nov. 15, 1924.

Power O'Malley. Int. Studio 49 : sup. 48-9, Apr., 1913.

Willard Paddock. Amer. Mag. of Art 7: 328-31, June, 1916. Int. Studio 46: sup. 66-9, May, 1912.

William M. Paxton. Int. Studio 39: sup. 46-8, Dec., 1909. *World Today $9: 1210-14$, Nov., 1905.

$\dagger$ Joseph T. Pearson.

Van Deering Perrine. Arts and Dec. 21: 14-15, May, 1924. Century $72: 165-71$, Sept., 1906. Craftsman 12:486-96, Aug., 1907. Cur. Lit. 41:407-10, Oct., 1906. *Touchstone 6:8-9, Oct., 1919.

Lilla Cabot Perry. Book: Clement. Magazine: Amer. Mag. of Art 14:600-4, Nov., 1923.

Jane Peterson. Washington Star, Apr. 8, 1924, p.9.

† Marjorie Phillips. 
Charles A. Platt. Book: *Monograph on the work of Charles A. Platt, with introd. by Royal Cortissoz, 1913. Magazines: Arch. Rec. 15:181-244, Mar., 1904. Arch. Rec. 36:81-97, Aug., 1914. Arch. Rec. 48:178-91, Sept., 1920. Arch. Rec. 51:16-32, Jan., 1922. *Garden Mag. $34: 250-3$, Jan., 1922. Int. Studio $82: 180-5$, Dec., 1925.

Abram Poole. *Colour n.s. 1 :10, Jan., 1925.

Hovsep Pushman. Art and Archaeology 10:194-5, Nov., 1920. *Fine Arts J. 34:79-84, Feb., 1916. Int. Studio 61: sup. 37-43, Apr., 1917. Int. Studio 67: sup. 79-81, May, 1919.

Grace Ravlin. Amer. Mag. of Art 13:164-5, May, 1922.

Edward W. Redfield. Amer. Mag. of Art $16: 3-8$, Jan., 1925. Arena $36: 20-6$, July, 1906. Arts and Dec. 6:135, Jan., 1916. Country Life $13: 194-7$, Dec., 1907 . Country Life $38: 38-9$, June, 1920. Int. Studio 41: sup. 29-36, Aug., 1910 . Int. Studio $75: 402-$ 10, Aug., 1922.

William Ritschel. *Fine Arts J. 34:275-80, June, 1916. Int. Studio 28: sup. 50-1, June, 1906.

†Henry R. Rittenberg.

Julius Rolshoven. Amer. Mag. of Art 11: 359-60, Aug., 1920. 1921.

Carl Rungius. Country Life 40:34-9, Sept.,

Chauncey F. Ryder. *Art Review 22:1, Sept., 1911.

\section{$\dagger$ Robert K. Ryland.}

Eugene F. Savage. Amer. Mag. of Art 15: 132-3, Mar., 1924. Arts and Dec. $20: 24-5$, Jan., 1924. Int. Studio $79: 2-8$, Apr., 1924. 
W. Elmer Schofield. Book: Isham. Magazines: Arts and Dec. 1:473-5, Oct., 1911. Int. Studio $48: 280-6$, Feb., 1913.

James Scott. *Art in America $12: 229-34$, Aug., 1924.

Leopold Seyffert. Book: Bryant-American pictures and their painters, (WP83-B844).

$\dagger$ John Sharman.

Henry B. Snell. Books: Hartmann, Isham. †Arthur Spear.

Robert Spencer. Int. Studio 76:485-91, Mar., 1923.

Maurice Sterne. Cur. Opinion 59:425-7, Dec., 1915. Int. Studio 46: sup. 3-13, Mar. 1912. Int. Studio 61: sup. 53-6, Apr., 1917. New Repub. 33:149, Jan. 3, 1923.

Albert Sterner. Bookman 10:550-4, Feb., 1900. Int. Studio 13:78-80, Mar,, 1901. Int. Studio 52: sup. 71-8, May, 1914. Outlook 137:99-102, May 21, 1924. Print Col. Q. 6: 213-24, Apr., 1916. Putnam's 2:387-99, July, 1907.

\section{†Alice Kent Stoddard.}

Gardner Symons. *Fine Arts J. 34:557-65, Nov., 1916. Outlook 105:881-7, Dec. 27, 1913.

Augustus Vincent Tack. Cur. Opinion 58: 269-70, Apr., 1915. Int. Studio 54: sup. 3-10, Nov., 1914. Int. Studio 58: sup. 19-24, Mar., 1916.

Henry O. Tanner. Int. Studio 50: sup. 1115, July, 1913. 
Edmund C. Tarbell. Book: Hartmann. Magazines: Amer. Mag. of Art $9: 217-28$, Apr., 1918. Arts and Dec. $2: 129-31$, Feb., 1912. Harper's 117:65-74, June, 1908. Int. Studio 32: sup. 71-87, Sept. 1907. *Mentor 8:26-7, Dec., 1920. *World Today 11:1077-85, Oct., 1906.

\section{†Charles Jay Taylor.}

\section{†Leslie P. Thompson.}

Giovanni B. Troccoli. House Beautiful 43: 13-15, Dec., 1917.

Allen Tucker. Int. Studio 52: sup. 19-20 Mar., 1914.

Helen M. Turner. Art World 3:490, Mar., 1918.

Walter Ufer. Amer. Mag. of Art $13: 507-$ 14, Dec., 1922. Art and Archaeol. 16:66-74, Aug., 1923. *Fine Arts J. 34:221-6, May, 1916. Int. Studio 77:293-9, July, 1923.

Douglas Vo!k. Amer. Mag. of Art 14:1415, Jan., 1923. Art World $3: 282-3$, Jan., 1918. Century 68: 654-5, Aug., 1904.

Robert Vonnoh. Int. Studio 54: sup. 4852, Dec., 1914. Int. Studio $77: 229-33$, June, 1923.

\section{†Fred Wagner.}

Horatio Walker. Books: Caffin-American masters of painting (W10-9C112), Hartmann, Isham. Magazines: Arts and Dec. 1:63-5, 88, Dec., 1910. Canad. Mag. 18:496-500, Apr., 1902. Canad. Mag. 53:21-9, May, 1919. Craftsman 14:138-45, May, 1908. Harper's 117:947-56, Nov., 1908. Int. Studio $77: 358$ 63, Aug., 1923. Lit. Dig. 78:31, Aug. 11, 1923. *Touchstone 5:482-5, Sept., 1919.

Martha Walter. Int. Studio 52: sup. 42-5, Apr., 1914. 
Frederick J. Waugh. Amer. Mag. of Art 13:15-18, Jan., 1922. Arts and Dec. 1:111-3, Jan., 1911. Century 100:641-8, Sept., 1920. Int. Studio 51:273-8, Feb., 1914. Int. Studio 60 : sup. 50-2, Dec., 1916 . Int. Studio 74 : sup. 125-35, Dec., 1921.

Irving R. Wiles. Art and Prog. 6:213-14, May, 1915. Arts and Dec. 1:402-4, Aug., 1911. Craftsman 18:347-53, June, 1910. Harper's $109: 802-3$, Oct., 1904. Int. Studio $77: 256-62$, June, 1922. Rev. of Rev. $34: 40-2$, July, 1906.

F. Ballard Williams. Amer. Mag. of Art 13:462-3, Nov., 1922. Int. Studio 42: sup. 53-60, Jan., 1911.

Charles H. Woodbury. Art and Prog. 4: 761-6, Nov., 1912. *Brush and Pencil 6:1-12, Apr., 1900. Int. Studio 42: sup. 71-8, Feb., 1911.

\section{†Stanley W. Woodward.}

Charles Morris Young. Int. Studio 28:36970, June, 1906.

The Public Library has published several reference lists on the history of painting and on various phases of the fine arts which are still available. The assistants in the reference room are always glad to help students of art history or criticism to locate needed material. 
THE PUBLIC LIBRARY OF THE DISTRICT OF COLUMBIA REFERENCE LIST NO. 22 .

\section{CONTEMPORARY AMERICAN PAINTERS}

INCLUDED IN THE ELEVENTH EXHIBITION OF CONTEMPORARY AMERICAN OIL PAINTINGS

THE CORCORAN GALLERY OF ART OCTOBER 28 - DECEMBER 9, 1928 INCLUSIVE

ISSUED WITH THE COOPERATION OF THE CORCORAN GALLERY OF ART

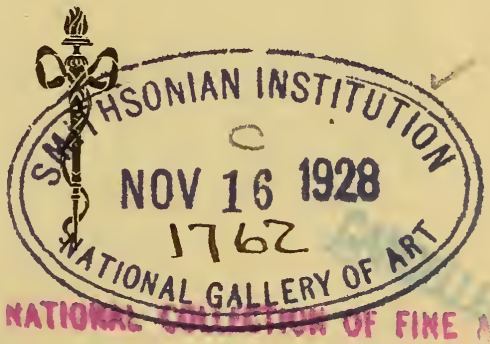

UNITED STATES

GOVERNMENT PRINTING OFFICE WASHINGTON 


\section{CONTEMPORARY AMERICAN PAINTERS}

Brief biographical information in regard to most of the painters exhibiting is given in Who's Who in Art, 1927, and Biographical Sketches of American Artists published by the Michigan State Library. Critical comments on many of the exhibitors are in A Collection in the Making by Duncan Phillips. When an artist is represented in these reference works or by slight mention in any of the other books included in the list, no specific note is made.

A valuable clipping file of material on American artists is maintained by the Corcoran Gallery of Art. Nearly all of the artists exhibiting are represented in this collection, which may be consulted by persons interested. A dagger $(\dagger)$ in front of a name indicates that material about the artist has been found only in this file.

Under each exhibitor (so far as he has been written about) references are given to material in the books, referred to only by their authors, given at the beginning of the list. In cases where a number of articles have been written about an artist a selection has been made of the ones which seem to be the best.

The items marked with a star (*) are not in the Public Library but may be found at the Library of Congress. All other books and periodicals referred to in this list may be found in the Public Library, Eighth and K Streets, NW.

The Public Library has published several reference lists on various phases of the fine arts which are still available. The assistants in the Art Division and the Reference Room are always glad to help students of art history or criticism to locate needed material. 


\section{BOOKS}

Caffin, C. H. Story of American painting. 1907. WP83-C11.

Clement, Mrs. C. E. Women in the fine arts. 1904. W10-9C59w.

Cortissoz, Royal. American artists. 1923. W83C817.

Hartmann, Sadakichi. History of American art. 2 v. 1902. W83-H255.

Innes, Mary. Schools of painting. 1911. WPIn65s.

Isham, Samuel. History of American painting, revised edition with supplementary chapters by Royal Cortissoz. 1927. WP83-Is3h.

Michigan State Library. Biographical sketches of American artists. 5th ed. rev. 1924. W10-9M 584 reference.

Phillips, Duncan. A collection in the making. 1926. + WC859-P54 reference.

Who's Who in Art (in American art annual, 1927). W-7Am reference.

\section{ARTISTS IN EXHIBITION}

Wayman Adams. Int. Studio, 77: 86-91, May, 1923.

Karl Anderson. Int. Studio, 76: 132-4, Nov. 1922.

William Auerbach-Levy. Survey, 32: 193, May $16,1914$.

†Burtis Baker.

†Frederick Clay Bartlett.

Gifford Beal. Int. Studio, 44: sup. 20-30, Aug. 1911; Int. Studio, 77: 236-42, June, 1923; *Touchstone, 5: 240-3, June, 1919. 
Cecilia Beaux. Books: Clement, Hartmann, Isham. Magazines: Amer. Mag. 96: 61-3, Oct. 1923; Art World, 3: 489-90, Mar. 1918; Century, 80: 581-7, Aug. 1910; Ind. 103: 382-4, Sept. 25, 1920; Int. Studio, 8: 215-22, Oct. 1899; Int. Studio, 41: sup. 3-10, July, 1910; Int. Studio, 85: 74-80, Oct. 1926; *Mentor, 12: 30-44, Oct. 1924; Scribner's, 22: 477-85, Oct. 1897; Woman Cit. n. s. 9: 12-13, Feb. 7, 1925.

Gerrit A. Beneker. Ind. 113: 43-4, July 19, 1924; Int. Studio, 73: sup. 63-6, Apr. 1921; Lit. Dig. 85: 28-9, June 20, 1925; *Mentor, 13: 43-50, Sept. 1925; Scribner's, 74: 290-300, Sept. 1923.

- Frank W. Benson. Book: Isham. Magazines: Amer. Mag. of Art, 9: 309-16, June, 1918; Amer. Mag. of Art, 12: 365-72, Nov. 1921; Art and Archaeol. 15: 92-5, Feb. 1923; Arts and Dec. 1: 195-7, Mar. 1911; Country Life, 36: 53-5, Oct. 1919; Harper's 119: 105-14, June, 1909; Ind. 113: 481-4, Dec. 6, 1924; Int. Studio, 35: sup. 99-104, Oct. 1908; Int. Studio, 74: 95-100, Oct. 1921; Lad. H. J. $44: 16-17$, Oct. 1927.

Oscar E. Berninghaus. Country Life, 33: 57-9, Apr. 1918.

Charles Bittinger. Int. Studio, 59: sup. 41-4, Aug. 1916.

Ernest L. Blumenschein. Outlook, 134: 504-7, Aug. 1, 1923.

Adolphe Borie. *Touchstone, 4: 519-21, Mar. 1919.

$\dagger$ Frederick A. Bosley.

Hugh H. Breckenridge. Amer. Mag. of Art, 7: 85-6, Jan. 1916; Int. Studio, 37: sup. 34-6, Mar. 1909.

$\dagger R$. Sloan Bredin.

Roy Brown. Int. Studio, 83: 78-82, Jan. 1926. George Elmer Browne. Amer. Mag. of Art, 17: 569-76, Nov. 1926; Int. Studio, 36: 286-92, Feb. 1909.

Charles Burchfield. Arts, 14: 5-12, July, 1928; Int. Studio, 80: 466-70, Mar. 1925. 
Bryson Burroughs. Book: Isham. Magazine: Arts and Dec. 12: 82-4, Dec. 28, 1919.

I. H. Caliga. New Eng. Mag. n. s. 32: 110, Mar. 1905.

†Arthur B. Carles.

Emil Carlsen. Book: Isham. Magazines: Int. Studio, 61: sup. 105-10, June, 1917; Int. Studio, 75: 300-8, July, 1922; Int. Studio, 88: 53-60, Oct. 1927; Lad. H. J. 44: 10, Dec. 1927; Scribner's 66: 767-70, Dec. 1919; Touchstone, 7: 110-16, May, 1920.

John Carroll. *Colour, 13: 33, Sept. 1920; Int. Studio, 83: 75-9, Feb. 1926.

James Chapin. Int. Studio, 83: 75-9, Apr. 1926; Survey, 60: 464-7, Aug. 1, 1928.

†Charles S. Chapman.

John E. Costigan. Int. Studio, 80: 426-32, Mar. 1925.

E. Irving Couse. Amer. Mag. of Art, 11: 400-5, Sept. 1920; Century, 99: 369-76, Jan. 1920; Craftsman, 18: 619-25, Sept. 1910; Outlook, 134: 504-7, Aug. 1, 1923; Southern Workm. 57: 17-23, Jan. 1928.

†Catharine C. Critcher.

Andrew M. Dasburg. *Arts, 6: 18-26, July, 1924.

Arthur B. Davies. Books: Cortissoz, Hartmann, Huneker-Pathos of distance (Y-H894p), Isham, *Phillips Memorial Art Gallery-Arthur B. Davies, 1924, Sherman-American painters of yesterday and to-day (W10-9Sh56). Magazines: Art and Archaeol. 4: 169-77, Sept. 1916; *Art in Amer. 6: 295-9, Oct. 1918; Arts and Dec. 8: 34, 36-7, Nov. 1917; Cur. Opinion, 64: 203-5, Mar. 1918; Forum, 51: 770-2, May, 1914; Int. Studio, 72: sup. 126-30, Feb. 1921; Int. Studio, 75: 212-19, June, 1922; Int. Studio, 77: 254-5, June, 1923; Lit. Dig. 56: 23-4, Jan. 26, 1918; New Repub. 13: 338, Jan. 19, 1918; *Print Connoisseur 3: 194-213, July, 1923; Scribner's, 80: 344-52, Sept. 1926; Touchstone, 6: 277-84, Feb. 1920. 
Charles H. Davis. Books: Hartmann, Isham. Magazines: Int. Studio, 75: 176-83, June, 1922; New Eng. Mag. n. s. 27: 423-37, Dec. 1902.

†Sidney E. Dickinson.

Paul Dougherty. *Art Review, 23: 1-3, Nov. 1911; Arts and Dec. 8: 251-4, Apr. 1918; Int. Studio 30: 180-2, Dec. 1906; Int. Studio, 36: sup. 3-4, Nov. 1908; Int. Studio, 73: sup. 54-8, Apr. 1921.

Guy Pene Du Bois. Arts and Dec. 4: 13-16, Nov. 1913.

W. Herbert Dunton. Amer. Mag. of Art, 15: 501-8, Oct. 1924.

†Jerry Farnsworth.

Truman E. Fassett. *Colour, 18: 85, May, June, 1923.

Nicolai Fechin. *Amer. Art News, 10: 1, Dec. 30, 1911.

$\dagger$ Gertrude Fiske.

John F. Folinsbee. Int. Studio, 73: sup. 38, Apr. 1921; Int. Studio, 76: 420-6, Feb. 1923.

Will Howe Foote. *Arts, Aug.-Sept. 1921.

Frederick C. Frieseke. Art and Prog. 3: 747-9, Oct. 1912; Arts and Dec. 3: 13-15, Nov. 1912; Int. Studio, 53: 259-64, Oct. 1914; Scribner's, 58: 643-6, Nov. 1915.

Maurice Fromkes. Art and Archaeol. 19: 162-74, Apr. 1925; Arts and Dec. 22: 34-5, Dec. 1924; Century, 77: 958, Apr. 1909; Country Life, 47: 86-90, Nov. 1924; Int. Studio, 80: 396-403, Feb. 1925; *Touchstone, 3: 302-8, July, 1918.

Daniel Garber. *Maitres Contemporains, 59: 10, 1912.

William J. Glackens. Book: Isham. Magazines: Amer. Mag. of Art, 7: 261-3, May, 1916; Arts, 3: 246-60, Apr. 1923; Arts and Dec. 4: 404-6, Sept. 1914; Arts and Dec. 7: 85, Dec. 1916; Arts and Dec. 14: 103, Dec. 1920; Int. Studio, 40: sup. 68, May, 1910; Touchstone, 7: 191-9, June, 1920.

†Walter Goltz.

†A. H. Gorson. 
Walter Griffin. Int. Studio 62: sup. 45-8, Aug. 1917.

Albert L. Groll. *Brush and Pencil, 11: 36-41, 1902; *Brush and Pencil, 18: 43-51, Aug. 1906; Century, 100: 385-92, July, 1920; Craftsman, 9: 826-36, Mar. 1906; Int. Studio, 28: sup. 78-82, May, 1906; Int. Studio, 29: 270-1, Sept. 1906; Int. Studio, 64: sup. 105-10, June, 1918; Int. Studio, 75: 496-501, Sept. 1922.

Lilian Westcott Hale. Amer. Mag. of Art, 18: 58-70, Feb. 1927.

Philip L. Hale. Amer. Mag. of Art, 7: 259-60, May, 1916; *World To-day, 14: 59-67, Jan. 1908.

Samuel Halpert. Int. Studio, 75: 144-50, Apr. 1922

George Harding. Amer. Mag. of Art, 10: 451-6, Oct. 1919; Int. Studio, 62: sup. 16-18, July, 1917.

Childe Hassam. Books: Caffin, Cortissoz, Hartmann, McSpadden, J. W.-Famous painters of America (W10-9M246), Magazines: Art and Prog. 2: 137-9, Mar. 1911; Int. Studio, 45: sup. 29-30, Dec. 1911; Int. Studio, 57: sup. 83-6, Jan. 1916; Int. Studio, 77: 2-7, Apr. 1923. Nation, 101: 698-9, Dec. 9, 1915; *Touchstone, 5: 272-80, July, 1919.

Charles W. Hawthorne. Art and Prog. 4: 821-5, Jan. 1913; Int. Studio, 26: 261-4, Sept. 1905; Int. Studio, 37; sup. 65-8, May 1909; Int. Studio, 61: sup. 19-24, Mar. 1917; Lit. Dig. 91: 27; Nov. 6, 1926.

Robert Henri. Books: Cortissoz, Hartmann, Henri-Art spirit (W-H397), Robert Henri, comp. by Pousette-Dart (W10-H397), *Yarrow, ed.Robert Henri, his life and works, 1921. Magazines: Amer. Mag. of Art, 7:473-9, Oct. 1916; Arts and Dec. 2: 213-5, Apr. 1912; Arts and Dec. 14: 36, Nov. 1920; Arts and Dec. 20: 26, Dec. 1923; Craftsman, 27: 459-69, Feb. 1915; Cur. Opinion, 71: 793-6, Dec. 1921; Ind. 64: pt. 2: 1427-32, June 25, 1908; Int. Studio, 56: 239-41, Oct. 1915; Lit. Dig. 80: 27-8, Jan. 5, 1924; Survey, 50: 279-82, June 1, 1923; *Touchstone, 5: 212-9, June, 1919. 
Eugene Higgins. Arts and Dec. 5: 134-5, 164-5, Feb. 1915; Craftsman, 12: 134-46, May, 1907; Cur. Lit. 43: 401-4, Oct. 1907; *Fine Arts J., Oct. 1907; Survey, 59: 562-3, Feb. 1, 1928; *Touchstone, 5: 360-9, Aug. 1919.

†Victor Higgins.

William H. Holmes. Art and Prog. 6: 11-14, Nov. 1914; Science n. s. 64: 571-2, Dec. 10, 1926.

Charles Hopkinson. Nation, 92: 369-70, Apr. $13,1911$.

Edward Hopper. *Arts, 5: 323, June, 1924; Arts, 11: 134-8, Mar. 1927; *Shadowland, Oct. 1922, pp. 22-3.

$\dagger$ Ernest L. Ipsen.

†Alexander R. James.

John C. Johansen. Amer. Mag. of Art, 10: 290-5, June, 1919; Int. Studio, 26: 264-7; Sept. 1905; Int. Studio, 42: sup. 3-6, Nov. 1910.

Bernard Karfiol. *Arts 6: 76-82, Aug. 1924.

†William Kaula.

Sergeant Kendall. Harper's, 117: 568-77, Sept. 1908.

Carl R. Krafft. Amer. Mag. of Art, 17: 475-80, Sept. 1926; *Fine Arts J. 34: 465-72, Oct. 1916.

Leon Kroll. Int. Studio, 72: 36-8, Feb. 1921.

Louis Kronberg. Amer. Mag. of Art, 19: 194-200, Apr. 1928; Int. Studio, 28: 74-5, Mar. 1906; New Eng. Mag. n. s. 38: 498-9, June, 1908.

Max Kuehne. Arts and Dec. 15: 31, May, 1921; Int. Studio, 68: sup. 37-40, Aug. 1919; Int. Studio, 79: 58-62, Apr. 1924: Int. Studio, 84:- 71-5, July, 1926.

William L. Lathrop. Amer. Mag. 104: 34-5, Nov. 1927; Int. Studio, 78: 132-8, Nov. 1923.

Ernest Lawson. Amer. Mag. of Art, 8: 257-63, May, 1917; *Art in Amer. 8: 32-9, Dec. 1919; Arts and Dec. 6: 505-7, Sept. 1916; Arts and Dec. 10: 257-8, Mar. 1919; Cur. Lit. 42: 406-9, Apr. 1907; Int. Studio, 59: sup. 13-15, July, 1916; Int. Studio, 72: sup. 114-17, Feb. 1921; Int. Studio, 78: 366-70, Feb. 1924. 
Hayley Lever. Int. Studio, 52: sup. 92-3, May 1914; Int. Studio, 70: sup. 67-70, May, 1920.

Jonas Lie. Amer. Mag. of Art, 16: 58-66, Feb. 1925; Arts and Dec. 12: 91, Dec. 1919; Arts and Dec. 15: 221, Aug. 1921; Country Life, 36: 55-7, July 1919; Craftsman, 13: 135-9, Nov. 1907; Cur. Lit. 52: 222-4, Feb. 1912; Int. Studio, 51: sup. 192-4, Feb. 1914; Int. Studio, 82: 102-7, Nov. 1925; Pan Amer. Union Bul. 38: 679-83, May, 1914.

Philip Little. Art and Prog. 5: 19-21, Nov. 1913. George Luks. Touchstone, 8: 32-8, Oct. 1920.

Walter MacEwen. Book: Isham. Magazine: * Maitres Contemporains, 46: 8, 1908.

\section{$\dagger$ Henry L. McFee.}

M. Jean MacLane (Mrs. John C. Johansen). Art and Prog. 3: 612, June, 1912; Arts and Dec. 3: 299-301, July, 1913; Harper's, 118: 291-3, Jan. 1909.

Ernest L. Major. Century, 42: 229, 318, June, 1891.

Gari Melchers. Books: Caffin, Isham. Magazines: Amer. Mag. of Art, 13: 117-21, Apr. 1922; Amer. Mag. of Art, 15: 79-85, Feb. 1924; Arts and Dec. 24: 40-1, Nov. 1925; Harper's, 114: 430-9, Feb. 1907; Int. Studio, 31: sup. 11-18, Mar. 1907; Int. Studio, 48; sup. 27-30, Dec. 1912; World's Work, 15: 10092-105, Apr. 1908.

tRichard S. Meryman.

Kenneth Hayes Miller. New Repub. 27: 2445, July 27, 1921.

Richard E. Miller. Art and Prog. 6: 39-40, 54-7, Dec. 1914; Int. Studio, 43: 263-73, June, 1911.

$\dagger$ Ross E. Moffett.

F. Luis Mora. Lit. Dig. 64: 40-1, Mar. 27, 1920.

H. Dudley Murphy. *Sketch Book, 6: 303-6, Nov. 1907.

Jerome Myers. Amer. Mag. of Art, 8: 481-5, Oct. 1917; Art. and Prog. 5: 89-94, Jan. 1914; Arts and Dec. 10: 257, 259, Mar. 1919; Craftsman, 29: 25-32, Oct. 1915; Int. Studio, 57: sup. 125-8, 
Feb. 1916; Survey, 51: 33-9, Oct. 1, 1923; *Touchstone, 5: 396-9, Aug. 1919.

†Hobart Nichols. Washington Star. Apr. 20, 1924 , pt. 3, p. 5.

Robert H. Nisbet. Int. Studio, 49: sup. 11-14, Mar. 1913.

John Noble. Amer. Mag. 104: 34-5, Aug. 1927; Int. Studio, 77: 457-65, Sept. 1923.

George L. Noyes. Int. Studio, 52: sup. 48-9, Apr. 1914.

$\dagger$ Marie Danforth Page.

William M. Paxton. Int. Studio, 39: sup. 46-8, Dec. 1909, *World Today, 9: 1210-14, Nov. 1905.

Van Deering Perrine. Arts and Dec. 21: 14-15, May, 1924; Century, 72: 165-71, Sept. 1906; Craftsman, 12: 486-96, Aug. 1907; Cur. Lit. 41: 407-10, Oct. 1906; *Touchstone 6: 8-9, Oct. 1919.

Lilla Cabot Perry. Book: Clement. Magazines: Amer. Mag. of Art, 14: 600-4, Nov. 1923; Amer. Mag. of Art, 18: 119-26, Mar. 1927.

Charles A. Platt. Book: *Monograph on the work of Charles A. Platt, with introd. by Royal Cortissoz, 1913. Magazines: Arch. Rec. 15: 181244, Mar. 1904; Arch. Rec. 36: 81-97, Aug. 1914; Arch. Rec. 48: 178-91, Sept. 1920; Arch. Rec. 51: 16-32, Jan. 1922; *Garden Mag. 34: 250-3, Jan. 1922; Int. Studio, 82: 180-5, Dec. 1925.

Abram Poole. *Colour n. s. 1: 10, Jan. 1925.

Edward W. Redfield. Amer. Mag. of Art, 16: 3-8, Jan. 1925; Arena, 36: 20-6, July, 1906; Arts and Dec. 6: 135, Jan. 1916; Country Life, 13: 194-7, Dec. 1907; Country Life, 3S: 38-9, June, 1920; Int. Studio, 41: sup. 29-36, Aug. 1910; Int. Studio, 75: 402-10, Aug. 1922; Lit. Dig. 92: 29, Jan. 22, 1927.

†Charles Rosen.

Albert Rosenthal. *Wilson's Photo. Mag. 49: 310-15, July, 1912.

Carl Rungius. Country Life, 40: 34-9, Sept. 1921.

Eugene F. Savage. Book: Isham. Magazines: Amer. Mag. of Art, 15: 132-3, Mar. 1924; Arts and Dec. 20: 24-5, Jan. 1924; Int. Studio, 79: 2-8, Apr. 1924; Scribner's, 82: 120-8, July, 1927. 
W. Elmer Schofield. Book: Isham. Magazines: Arts and Dec. 1: 473-5, Oct. 1911; Int. Studio, 48: 2S0-6, Feb. 1913.

Leopold Seyffert. Book: Bryant-American pictures and their painters (WP83-B844).

†John Sharman.

John Sloan. Book: Caffin. Magazines: Arts, 11: 168-78, Apr. 1927; Craftsman, 15: 559-64, Feb. 1909; Harper's Weekly, 58: 20-1, Nov. 22, 1913; Int. Studio, 58: sup. 25-8, Mar. 1916; Lit. Dig. 97: 26-7, Apr. 21, 1928; *Touchstone, 4: 36270, Feb. 1919; Va. Q. R. 1: 196-204, July, 1925.

Henry B. Snell. Books: Hartmann, Isham.

$\dagger$ Alice Ruggles Sohier.

tArthur P. Spear.

Eugene Speicher. *Arts, 6: 314-27, Dec. 1924; Int. Studio, 46: sup. 19-22, Mar. 1912.

Robert Spencer. Int. Studio, 76: 485-91, Mar. 1923.

Maurice Sterne. Cur. Opinion, 59:425-7, Dec. 1915; Int. Studio, 46: sup. 3-13, Mar. 1912; Int. Studio, 61: sup. 53-6, Apr. 1917; New Repub. 33: 149, Jan. 3, 1923; New Repub. 45: 355, Feb. 17, 1926.

†Alice Kent Stoddard.

Gardner Symons. *Fine Arts J. 34: 557-65, Nov. 1916; Outlook, 105: 881-7, Dec. 27, 1913.

Augustus Vincent Tack. Cur. Opinion, 58: 26970, Apr. 1915; Int. Studio, 54: sup. 3-10, Nov. 1914; Int. Studio, 58: sup. 19-24, Mar. 1916.

Henry O. Tanner. Int. Studio, 50: sup. 11-15, July, 1913.

†Leslie P. Thompson.

Allen Tucker. Arts, 13: 74-88, Feb. 1928; Int. Studio, 52: sup. 19-20, Mar. 1914.

Helen M. Turner. Art World, 3: 490, Mar. 1918.

Walter Ufer. Amer. Mag. of Art, 13: 507-14, Dec. 1922; Art and Archaeol. 16: 66-74, Aug. 1923; *Fine Arts J. 34: 221-6, May, 1916; Int. Studio, 77: 293-9, July, 1923; Lit. Dig. 97: 25, May 19, 1928. 
Horatio Walker. Books: Caffin-American masters of painting (W10-9C112), Hartmann, Isham. Magazines: Arts and Dec. 1: 63-5, 88, Dec. 1910; Canad. Mag. 18: 496-500, Apr. 1902. Canad. Mag. 53: 21-9, May, 1919; Craftsman, 14: 138-45, May, 1908; Harper's, 117: 947-56, Nov. 1908; Int. Studio, 77: 358-63, Aug. 1923; Lit. Dig. 78: 31, Aug. 11, 1923; *Touchstone, 5: 482-5, Sept. 1919.

Frederick J. Waugh. Amer. Mag. of Art, 13: 15-18, Jan. 1922; Arts and Dec. 1: 111-13, Jan. 1911; Century, 100: 641-8, Sept. 1920; Gard. and Home B. 46: 138-9, Oct. 1927; Int. Studio, 51: 273-8, Feb. 1914; Int. Studio, 60: sup. 50-2, Dec. 1916; Int. Studio, 74: sup. 125-35, Dec. 1921.

Irving R. Wiles. Art and Prog. 6: 213-14, May, 1915; Arts and Dec. 1: 402-4, Aug. 1911; Craftsman, 18: 347-53, June, 1910; Harper's, 109: 802-3, Oct. 1904; Int. Studio, 77: 256-62, June, 1922; Int. Studio, 88: 61-5, Nov. 1927; Rev. of Rev. 34: 40-2, July, 1906.

Charles H. Woodbury. Art and Prog. 4: 761-6, Nov. 1912; *Brush and Pencil, 6: 1-12, Apr. 1900; Int. Studio, 42: sup. 71-8, Feb. 1911.

tStanley W. Woodward. 




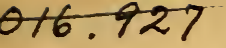

THE PUBLIC LIBRARY OF THE DISTRICT OF COLUMBIA REFERENCE LIST NO. 24

\section{CONTEMPORARY AMERICAN PAINTERS}

INCLUDED IN THE TWELFTH BIENNIAL EXHIBITION OF CONTEMPORARY AMERICAN OIL PAINTINGS

THE CORCORAN GALLERY OF ART NOVEMEER 30, 1930 - JANUARY 11, 1931 INCLUSIVE

ISSUED WITH THE COOPERATION OF THE CORCORAN GALLERY OF ART

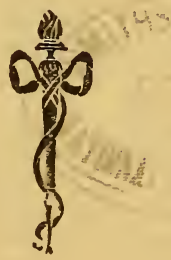

UNITED STATES

GOVERNMENT PRINTING OFFICE

WASHINGTON : 1930 


\section{CONTEMPORARY AMERICAN PAINTERS}

Brief biographical information in regard to most of the painters exhibiting is given in Who's Who in Art, 1929; Dictionary of American Painters, Sculptors, and Engravers, by Mantle Fielding; American Arts, by R. E. Jackson; The American Spirit in Art, by F. J. Mather, jr., and others; and Biographical Sketches of American Artists, published by the Michigan State Library. Critical comments on many of the exhibitors are in A Collection in the Making by Duncan Phillips. When an artist is represented in these reference works or by slight mention in any of the other books included in the list, no specific note is made.

A valuable clipping file of material on American artists is maintained by the Corcoran Gallery of Art. Nearly all of the artists exhibiting are represented in this collection, which may be consulted by persons interested. A dagger $(\dagger)$ in front of a name indicates that material about the artist has been found only in this file.

Under each exhibitor (so far as he has been written about) references are given to material in the books, referred to only by their authors, given at the beginning of the list. In cases where a number of articles have been written about an artist a selection has been made of the ones which seem to be the best.

The items marked with a star (*) are not in the Public Library but may nearly all be found at the Library of Congress. All other books and periodicals referred to in this list may be found in the Public Library, Eighth and K Streets NW.

The Public Library has published several reference lists on various phases of the fine arts which are still available. The assistants in the art division are always glad to help students of art history or criticism to locate needed material. 


\section{BOOKS}

Fielding, Mantle. Dictionary of American painters, sculptors, and engravers. 1926. W835F46. Reference.

Isham, Samuel. History of American painting, revised edition with supplementary chapters by Royal Cortissoz. 1927. WP83-Is3h.

Jackman, R. E. American arts. 1928. W83-J13.

La Follette, Suzanne. Art in America. 1929. W83-L13.

Lee, Cuthbert. Contemporary American portrait painters. 1929. WP83-L51.

Mather, F. J., jr., and others. The American spirit in art. (In the Pageant of America series.) 1927. F83-P144.

Michigan State Library. Biographical sketches of American artists. 5th ed. rev. 1924. W109 M584.

Phillips, Duncan. A collection in the making. 1926. WC859-P54.

Who's Who in Art. (In American art annual, 1929.) W-7Am. Reference.

\section{ARTISTS IN EXHIBITION}

Wayman Adams. Book: Lee. Magazines: Amer. Mag. 106: 24-7, Oct. 1928; Amer. Mag. of Art, 21: 640-8, Nov. 1930; Int. Studio, 77: 86-91, May, 1923; Lit. Dig. 100: 63, Jan. 5, 1929; Survey, 64: 318-19, July 1, 1930.

George G. Adomeit. *Art Dig. 4:15, Mar., 1930.

Ivan LeLorraine Albright. *Art. Dig. 5: 5, Oct. 1, 1930.

Karl Anderson. Book: Lee. Magazine: Int. Studio, 76: 132-4, Nov. 1922.

$$
\text { 23694-30 }
$$


† James E. Bailey.

tBurtis Baker.

†Jozef G. Bakos.

Kenneth Bates.

† Gustave Baumann.

Gifford Beal. Int. Studio, 44: sup. 20-30, Aug. 1911; Int. Studio, 77: 236-42, June, 1923; *Touchstone, 5: 240-3, June, 1919.

Oscar E. Berninghaus. Country Life, 33: 57-9, Apr. 1918.

Theresa Bernstein. Book: Bryant LorindaAmerican pictures and their painters (WP83-B844). Magazine: Int. Studio 66: sup. 97-102, Feb. 1919.

George Biddle. Amer. Mag. of Art, 20: 431-41, Aug. 1929.

Charles Bittinger. Book: Bryant LorindaAmerican pictures and their painters (WP83-B844). Magazine: Int. Studio, 59: sup. 41-4, Aug. 1916. Arnold Blanche. Art and Archaeol. 29: 39, Jan. 1930; Arts, 16: 419-21, Feb. 1930.

Ernest L. Blumenschein. Outlook 134: 504-7, Aug. 1, 1923.

Adolphe Borie. *Touchstone 4: 519-21, Mar. 1919.

†Frederick A. Bosley.

tRobert Brackman.

tEmile Branchard.

† Ross Braught.

Hugh H. Breckenridge. Amer. Mag. of Art, 7: 85-6, Jan. 1916. Int. Studio, 37: sup. 34-6, Mar. 1909.

†R. Sloan Bredin.

Alexander Brook. Art and Archaeol. 29: 34, 36, Jan. 1930; *Creative Art, 5: 713-18, Oct. 1929.

Roy Brown. Int. Studio, 83: 78-82, Jan., 1926.

Margaret Fitzhugh Browne. Book: Lee. Amer. Mag. of Art, 16: 408-11, Aug. 1925, *Art News, 28:13, Dec. 7, 1929. 


\section{3}

Edward Bruce. Amer. Mag. of Art, 20: 480-1, Aug. 1929; Amer. Mag. of Art, 21: 75-81, Feb. 1930; Arts, 7: 273-5, May, 1925; Int. Studio, 88: 57-62, Dec. 1927; Int. Studio, 94: 82, Nov. 1929.

†Buk.

Charles Burchfield. Arts, 14: 5-12, July, 1928; Int. Studio, 80: 466-70, Mar. 1925; Int. Studio, 95: 27, Jan. 1930; Survey, 61: 159-61, Nov. 1, 1928. Bryson Burroughs. Book: Isham. Magazine: Arts and Dec. 12: 82-4, Dec. 28, 1919.

Emil Carlsen. Book: Isham. Magazines: Int. Studio, 61: sup. 105-10, June, 1917; Int. Studio, 75: 300-8, July, 1922; Int. Studio, 88: 53-60, Oct. 1927, Lad. H. J. 44: 10, Dec. 1927; Scribner's 66: 767-70, Dec. 1919; Touchstone, 7: 110-16, May, 1920.

John F. Carlson. Book: Bryant Lorinda-American pictures and their painters (WP83-B844). Magazines: *Apollo 11; 376, May, 1930, Review of his book, Elementary principles of landscape painting (WPS-C19); Art News, 28: 12, Feb. 22, 1930; *Fine Arts J. 35: 203-6, Mar. 1917.

John Carroll. Art News, 28: 22, May 24, 1930; *Colour, 13: 33, Sept. 1920; Int. Studio, 83: 75-9, Feb. 1926.

Clarence Holbrook Carter. *Cleveland Mus. Bul. 17: 86, 96, May, 1930; *Rochester Bul. 2:10, Nov. 1929.

Francis Chapin. *Art Dig. 4: 6, Apr. 1, 1930.

James Chapin. Int. Studio, 83: 75-9, Apr. 1926; Survey, 60: 464-7, Aug. 1, 1928.

C. K. Chatterton. Amer. Mag. of Art. 16: 308-10, June, 1925; Art and Archaeol. 5: 289-93, May, 1917.

Glenn O. Coleman. Book: La Follette. Magazine: Arts, 14: 261-5, Nov. 1928.

John E. Costigan. Int. Studio 80: 426-32, Mar. 1925.

†Catharine C. Critcher. 


\section{4}

Charles C. Curran. Book: Lee. Magazines: Critic 48: 38-9, Jan. 1906; Lit. Digest, 102: 23, Sept. 7, 1929.

John Steuart Curry. Ar's, 16: 425, Feb. 1930; Survey, 64: 311-13, July 1, 1930.

Andrew Dasburg. *Arts, 6: 18-26, July, 1924.

Randall Davey. Amer. Mag. of Art, 7: 314-17, June, 1916; Int. Studio 75: 57-60, Mar. 1922.

Charles $\mathbf{H}$. Davis. Book: Isham. Magazines: Int. Studio, 75: 176-83, June, 1922; New Eng. Mag. n. s. 27:423-37, Dec. 1902.

†Julius Delbos.

F. Usher DeVoll. Amer. Mag. of Art, 21: 150, Mar. 1930.

Edwin W. Dickinson. Lit. Digest, 103: 21, Nov. $30,1929$.

Sidney E. Dickinson. Amer. Mag. of Art, 21: 394, July, 1930.

Paul Dougherty. *Art Review 23: 1-3, Nov. 1911; Arts and Dec. 8: 251-4, Apr. 1918; Int. Studio, 30: 180-2, Dec. 1906; Int. Studio, 36: sup. 3-4, Nov. 1908; Int. Studio, 73: sup. 54-8, Apr. 1921.

Guy Pene Du Bois. Book: La Follette. Magazines: *Art in Amer. 18: 25-8, Dec. 1929; Arts and Dec. 4: 13-16, Nov. 1913.

W. Herbert Dunton. Amer. Mag. of Art, 15: 501-8, Oct. 1924.

†Charles Warren Eaton.

† William J. Edmondson.

tWaiter Farndon.

Ernest Fiene. Arts, 16: 425, Feb. 1930.

†Gertrude Fiske.

John F. Folinsbee. Int. Studio, 73: sup. 38, Apr. 1921; Int. Studio, 76: 420-6, Feb. 1923.

Will Howe Foote. *Arts, Aug.-Sept., 1921.

Loren Ford. Survey, 64: 227-9, June 1, 1930. 
Frederick C. Frieseke. Art and Prog. 3: 747-9, Oct. 1912; Arts and Dec. 3: 13-15, Nov. 1912; Int. Studio, 53: 259-64, Oct. 1914; *Lad. H. J. 43: 16-17, July, 1926; Scribner's, 58: 643-6, Nov. 1915.

Maurice Fromkes. Art and Archaeol. 19: 162-74, Apr. 1925; *Art News, 28: 13, Dec. 7, 1929; Arts and Dec. 22: 34-5, Dec. 1924; Century, 77: 958, Apr. 1909; Country Life, 47: 86-90, Nov. 1924; Int. Studio, 80: 396-403, Feb. 1925; *Touchstone, 3: 302-8, July, 1918.

Daniel Garber. *Lad. H. J. 43: 20-1, May, 1926; * Maitres Contemporains 59: 10, 1912.

tS. Wood Gaylor.

Lillian Genth. Book: Bryant Lorinda-American pictures and their painters (WP83-B844). Magazines: Amer. Mag. of Art, 18: 227-37, May, 1927; *Fine Arts J. 37: 37-9, June, 1919; Int. Studio, 50: sup. 55-61, Oct. 1913; Lit. Dig. 100: 23, Mar. 16, 1929.

William Glackens. Books: Isham, La Follette. Magazines: Amer. Mag. of Art, 7: 261-3, May, 1916; Arts, 3: 246-60, Apr. 1923; Arts and Dec. 4: 404-6, Sept. 1914; Arts and Dec. 7: 85, Dec. 1916; Arts and Dec. 14: 103, Dec. 1920; *Detroit Inst. Bul. 12: 7-8, Oct. 1930; Int. Studio, 40: sup. 68, May, 1910; Touchstone, 7: 191-9, June, 1920.

† Sudduth Goff.

Anne Goldthwaite. Int. Studio, 59: sup. 3-8, July, 1916.

Harry Gottlieb. Arts, 15: 99-102, Feb. 1929.

†John R. Grabach.

$\dagger$ J. Jeffrey Grant.

$+W$. Granville-Smith.

†Edwin Booth Grossman.

tJohanna K. W. Hailman.

Lilian Westcott Hale. Amer. Mag. of Art, 18: 58-70, Feb. 1927. 
Philip L. Hale. Amer. Mag. of Art. 7: 259-60, May, 1916; *World Today, 14: 59-67, Jan. 1908.

George Harding. Amer. Mag. of Art 10: 451-6, Oct. 1919. Int. Studio 62: sup. 16-18, July, 1917.

Bertram Hartman. Book: La Follette.

Childe Hassam. Books: La Follette, McGuire, J. C.-Childe Hassam (In American Etchers, v. 3, W10-H277a); McSpadden, J. W.-Famous painters of America (WP83-M246); Poussette-Dart, Nathaniel, comp.-Childe Hassam (W10-H277, Ref.). Magazines: Art and Prog. 2: 137-9, Mar. 1911; Int. Studio, 45: sup. 29-30, Dec. 1911; Int. Studio, 57: sup. 83-6, Jan. 1916; Int. Studio, 77: 2-7, Apr. 1923; Int. Studio, 93: 78, June, 1924; Nation, 101: 698-9, Dec. 9, 1915; Scribner's 84: 415-22, Oct, 1928; Touchstone, 5: 272-80, July, 1919.

Charles W. Hawthorne. Books: Isham, Lee. Magazines: Art and Archaeol. 28: 87-8, Sept. 1929; Art and Prog. 4: 821-5, Jan. 1913; Int. Studio, 26: 261-4, Sept. 1905; Int. Studio, 37: sup. 65-8, May 1909; Int. Studio, 61: sup. 19-24, Mar. 1917; Lit. Dig. 91: 27; Nov. 6, 1926; Lit. Dig. 98: 30, Aug. 25, 1928.

\section{†Norbert Heermann. \\ † Martin Hennings.}

Henry Hensche. *Art Dig. 4: 6, Apr. 1, 1930.

Harry Hering. Arts, 16: 342, Jan. 1930. 1930.

Aldro T. Hibbard. Art News, 28: 15-16, Jan. 25,

Eugene Higgins. Arts and Dec. 5: 134-5, 164-5, Feb. 1915; Craftsman, 12: 134-46, May 1907; Cur. Lit. 43: 401-4, Oct. 1907; *Fine Arts J. Oct. 1907; Survey, 59: 562-3, Feb. 1, 1928; *Touchstone, 5: 360-9, Aug. 1919.

†Victor Higgins.

tClarence K. Hinkle.

Stefan Hirsch. *Newark Museum 2: 54, Apr. 1929. 
Charles Hopkinson. Book: Lee. Magazine: Nation 92: 369-70, Apr. 13, 1911.

Edward Hopper. Book: La Follette. Magazines: *Arts, 5: 323, June 1924; Arts, 11: 134-8, Mar. 1927; *Shadowland, Oct. 1922, pp. 22-3.

Ernest L. Ipsen. Book: Lee. Amer. Mag. of Art, 21: 195, Apr. 1930.

John C. Johansen. Book: Lee. Magazines: Amer. Mag. of Art 10: 290-5, June, 1919; Int. Studio, 26: 264-7, Sept. 1905; Int. Studio, 42: sup. 3-6, Nov. 1910 .

$\dagger$ J. Theodore Johnson.

Morris Kantor. Book: Phillips.

Bernard Karfiol. *Arts, 6: 76-82, Aug. 1924.

Sergeant Kendall. Book: Isham. Harper's, 117: 568-77, Sept. 1908.

Rockwell Kent. Books: Isham, Kent-Voyaging southward from the Strait of Magellan (G995T-K4), Wilderness (G945-K418). Magazines: Amer. Mag. 99: 18-21, Mar. 1925; *Architecture (N. Y.), 61: 285-8, May 1930; Arts and Dec. 11: 70-2, June, 1919; Arts and Dec. 12: 324-5, Mar. 1920; Country Life, 56: 35-8, July, 1929; Cur. Opinion, 62: 277-8, Apr. 1917; Cur. Opinion, 67: 52-3, July 1919; Cur. Opinion, 68: 681-5, May 1920; Int. Studio, 67: sup. 105-12, June, 1919; Int. Studio, 79: 272-6, July, 1924; Lit. Dig. 61: 31-2, May 31, 1919; Lit. Dig. 106: 17-18, Aug. 23, 1930; New Repub. 50: 302-3, May 4, 1927; Outlook, 155: 391-3, July 9, 1930; Wilson Bul. 4: 356, Apr. 1930.

Georgiana Klitgaard. Arts, 16: 118, Oct. 1929.

Karl Knaths. Art and Understanding, 1: 110-14, Nov. 1929; Art. News, 28: 12, Mar. 1, 1930.

Leon Kroll. *Art in Amer. 18: 299-303, Oct. 1930; *Canadian Bookman, 12: 39, Feb. 1930; Int. Studio, 72: 36-8, Feb. 1921. 
Louis Kronberg. Amer. Mag. of Art, 19: 194-200, Apr. 1928; Int. Studio, 28: 74-5, Mar. 1906; New Eng. Mag. n. s. 38: 498-9, June 1908.

Yasuo Kuniyoshi. Arts, 5: 22-7, Jan. 1924; Arts and Dec. 7: 251-3, Mar. 1917; Dial, 76: 295-7, Mar. 1924, *Trans-Pacific, 13: 10, Oct. 23, 1926.

† Richard Lahey.

William L. Lathrop. Amer. Mag. 104: 34-5, Nov. 1927; Int. Studio, 78: 132-8, Nov. 1923.

Ernest Lawson. Amer. Mag. of Art 8: 257-63, May 1917: *A.rt in Amer. 8: 32-39, Dec. 1919; Arts and Dec. 6: 505-7, Sept. 1916; Arts and Dec. 10: 257-8, Mar. 1919; *Canadian Bookman, 12:39, Feb. 1930; Cur. Lit. 42: 406-9, Apr. 1907; Int. Studio, 59: sup. 13-15, July 1916; Int. Studio, 72 : sup. 114-17, Feb. 1921; Int. Studio, 78: 366-70, Feb. 1924.

Jonas Lie. Book: Bryant Lorinda-American pictures and their painters (WP83-B844). Magazines: Amer. Mag. of Art, 16: 58-66, Feb. 1925; Art News, 29: 6, Oct. 11, 1930; Arts and Dec. 12: 91, Dec. 1919; Arts and Dec. 15: 221, Aug. 1921; Country Life, 36: 55-7, July 1919; Craftsman, 13: 135-9, Nov. 1907; Cur. Lit. 52: 222-4, Feb. 1912; Int. Studio, 51: sup. 192-4, Feb. 1914; Int. Studio 82: 102-7, Nov. 1925; Pan Amer. Union Bul. 38: 679-83, May 1914.

Luigi Lucioni. Art News, 28:10, Feb. 8, 1930.

George Luks. Books: La Follette, Lee. Magazines: Outlook, 154: 34, Jan. 1, 1930; Rev. of Revs. 80: 82-3, Sept. 1929; Survey, 55: 20-4, Oct. 1, 1925; Touchstone, 8: 32-8, Oct. 1920.

Stewart S. MacDermott. Art News, 28: 12, Jan. 4, 1930; *Creat. Art, 6: sup. 14, Jan., 1930; Int. Studio, 95: 76, Feb. 1930.

$\dagger$ Henry Lee McFee. 
M. Jean McLane. Book: Lee. Magazines: Art and Prog. 3: 612, June, 1912; Arts and Dec. 3: 299-301, July, 1913; Harper's 118: 291-3, Jan., 1909.

Emma Fordyce MacRae. *Art Digest, 4: 17, Mar. 15, 1930; Art News, 28: 10, Feb. 15, 1930.

Kristjan Magnusson. Amer. Mag. of Art, 21: 435-8, Aug. 1930.

$\nmid$ Peppino Mangravite.

$\dagger$ Alexis B. Many.

Henry Mattson. Arts 15: 26-9, Jan., 1929.

Gari Melchers. Books: Brinton, ChristianModern artists (W10-9B774), Isham, Lee. Magazines: Amer. Mag. of Art, 13: 117-21, Apr., 1922; Amer. Mag. of Art, 15: 79-85, Feb., 1924; Arts and Dec. 24: 40-1, Nov., 1925; Harper's, 114: 430-9, Feb., 1907; Int. Studio, 31: sup. 11-18, Mar., 1907; Int. Studio, 48; sup. 27-30, Dec. 1912; *Lad. H. J. 43: 26-7, Dec. 1926; Mentor, 17: 36-7, Aug., 1929; World's Work 15: 10092-105, Apr. 1908.

†Richard S. Meryman. 1930.

William Meyerowitz. *Art Digest, 4:22, Mar. 1,

Kenneth Hayes Miller. Books; *Goodrich, L.Kenneth Hayes Miller (pamphlet); La Follette. Magazines: *Art in Amer. 18: 86-92, Feb., 1930; *Art News, 28: 13, Dec. 7, 1929; Arts, 14: 300-7, Dec. 1928; Arts, 16: 641, May, 1930; New Repub. 27: 244-5, July 27, 1921.

Richard E. Miller. Art and Prog. 6: 39-40, 54-7, Dec., 1914; Int. Studio, 43: 263-73, June, 1911.

†Ross Moffett.

†A. Molarsky.

$\dagger$ Maurice Molarsky.

H. Dudley Murphy. *Sketch Book, 6: 303-6, Nov., 1907. 
Jerome Myers. Books: Caffin, C. H.-Story of American painting (WP83-C11), Isham, La Follette. Magazines: Amer. Mag. of Art, 8:481-5, Oct. 1917; Art and Prog. 5:89-94, Jan. 1914; Arts and Dec. 10:257, 259, Mar. 1919; Craftsman, 29:25-32, Oct. 1915; Int. Studio, 57: sup. 125-8, Feb. 1916; Survey, 51:33-9, Oct. 1, 1923; *Touchstone, 5:396-9, Aug. 1919.

Warren Newcombe. *Art Dig. 4: 18, Mar. 15, 1930.

John Noble. Amer. Mag. 104:34-5, Aug. 1927; Int. Studio, 77:457-65, Sept. 1923; Outlook, 155:153, May 28, 1930. 1926.

B. J. O. Nordfeldt. Survey, 57:132-3, Nov. 1,

Nura. Art News, 28:13, Apr. 19, 1930.

George Oberteuffer. Int. Studio, 43:275-80, June, 1911.

Ivan G. Olinsky. Art News, 29:11, Oct. 11, 1930; Lit. Dig. 83:29, Nov. 15, 1924.

$\dagger$ J. Olaf Olson.

William M. Paxton. Book: Lee. Magazines: Int. Studio, 39: sup. 46-48, Dec. 1909; Lit. Dig. 89:25, June 19, 1926; *World Today, 9:1210-14, Nov. 1905.

$\dagger$ Joseph T. Pearson.

Charles H. Pepper. *World Today, 9:1271-8, Dec. 1905.

Van Deering Perrine. Arts and Dec. 21:14-15, May, 1924; Century, 72:165-71, Sept. 1906; Craftsman, 12:486-96, Aug. 1907; Cur. Lit. 41:40710, Oct. 1906; *Touchstone, 6:8-9, Oct. 1919.

Marjorie Phillips. Book: La Follette. Magazine: Art News, 28:20, Mar. 22, 1930.

Salvatore Pinto. Art News, 28:10, Jan. 11, 1930.

Ogden M. Pleissner. Art News, 28:16, Jan. 11, 1930.

Joseph Pollet. *Creat. Art, 6:32-9, Jan. 1930 
Abram Poole. Art and Archaeol. 29:129, Mar. 1930; Arts and Dec. 23:54, Sept. 1925; *Colour, n. s. 1:10, Jan. 1925 .

Henry Rankin Poore. Book: Isham. Magazines: Amer. Mag. of Art, 21:543, Sept. 1930, Review of his book, *Art principles in practice for the student and layman in art"; Country Life 42:34-9, Sept. 1922; Dial, 35:174, Sept. 16, 1903, Review of his book, "Pictorial composition and the critical judgment of pictures" (W-P795); Int. Studio, 37: sup. 20-4, Mar. 1909; New Eng. Mag. n. s. 39:604-5, Jan. 1909.

M. Elizabeth Price. Art News, 28:10, Mar. 8, 1930.

† Norris Rahming.

Ellen Emmet Rand. Book: Lee. Magazine: Amer. Mag. of Art, 19:471-9, Sept. 1928.

Edward W. Redfield. Book: Isham. Magazines: Amer. Mag. of Art, 16:3-8, Jan. 1925; Amer. Mag. of Art, 21:139-42, Mar. 1930; Arena, 36: 20-6, July, 1906; Arts and Dec. 6:135, Jan. 1916; Country Life, 13:194-7, Dec. 1907; Country Life, 38:38-9, June, 1920; Int. Studio, 41:sup. 29-36, Aug. 1910; Int. Studio, 75:402-10, Aug. 1922; *Lad. H. J. 43:20-1, May, 1926; Lit. Dig. 92:29, Jan. 22, 1927.

†Umberto Romano.

tCharles Rosen.

† Margery Austen Ryerson.

† Margarett Sargent.

Charles Schlein. Art News, 28:15, May 10, 1930.

Henry Schnakenberg. *Art Digest, 4:6, Apr. 15, 1930; Int. Studio, 84:58-62, Aug. 1926.

W. Elmer Schofield. Book: Isham. Magazines: Arts and Dec. 1:473-5, Oct. 1911; Int. Studio, 48:280-6, Feb. 1913. 
Leopold Seyfiert. Books: Bryant LorindaAmerican pictures and their painters (WP83-B844), Lee. Magazines: Amer. Mag. of Art, 20:64-73, Feb. 1929; Int. Studio, 84: 84, July, 1926.

\section{†John Sharman.}

Edward Simmons. Books: Bryant LorindaAmerican pictures and their painters (WP83-B844), Isham, Simmons Edward-From 7 to 70 (W10-Si46). Magazine: Cur. Opinion, 74:58-60, Jan. 1923.

Gerrit V. Sinclair. *Milwaukee Inst. Bul. 3: 5, Apr. 1930.

John Sloan. Books: Gallatin, A. E. ed.-John Sloan (W10-Si52), La Follette. Magazines: Amer. Mag. of Art, 20:554-9, Oct. 1929, Amer. Mag. of Art, 21:282, May, 1930; Arts, 11:168-78, Apr. 1927; Craftsman, 15:559-64, Feb. 1909; Harper's Weekly, 58:20-1, Nov. 22, 1913; Int. Studio, 58: sup. 25-8, Mar. 1916; Lit. Dig. 97:26-7, Apr. 21, 1928; Theatre Arts Mag. 14:679-82, Aug. 1930; *Touchstone, 4:362-70, Feb. 1919; Va. Q. R. 1:196-204, July, 1925.

Eugene Speicher. Book: Bryant LorindaAmerican pictures and their painters (WP83-B844). Magazines:*Arts, 6: 314-27, Dec. 1924; Int. Studio, 46: sup. 19-22, Mar. 1912; Int. Studio, 95:26, Jan. 1930; New Repub. 58:280, Apr. 24, 1929.

$\dagger$ Francis Speight.

Robert Spencer. Int. Studio, 76:485-91, Mar. 1923.

Maurice Sterne. Book: Birnbaum, Martin-Introductions (W10-9B5361). Magazines: Art and Understanding, 1:86-9, Nov. 1929: Art News, 28:22, Feb. 1, 1930; Cur. Opinion, 59:425-7, Dec. 1915; Int. Studio, 46: sup. 3-13, Mar. 1912; Int. Studio 61: sup. 53-6, Apr. 1917; Int. Studio, 95:27, 35, 58, Jan. 1930; New Repub. 33:149, Jan. 3, 1923; New Repub. 45:355, Feb. 17, 1926; New Repub. 61:142-3, Dec. 25, 1929 
Albert Sterner. Books: Isham, Lee. Magazines: Art News 28:13, Apr. 5, 1930; Arts and Dec. 24:42-4, Feb. 1926; Bookman 10:550-4, Feb. 1900; Int. Studio, 13:78-80, Mar. 1901; Int. Studio, 52: sup. 71-8, May, 1914; Outlook 137:99-102, May 21, 1924; Print Col. Q. 6:213-24, Apr. 1916; Putnam's 2:387-99, July 1907.

Alice Kent Stoddard. Amer. Mag. of Art, 21: 480, Sept. 1930.

Anthony Thieme. Amer. Mag. of Art, 21:531, Sept. 1930; Lit. Dig. 103: 28, Oct. 5, 1929; *Wadsworth Ath. Bul. 8: 24, Apr. 1930.

$\dagger$ Leslie P. Thompson.

†Ernest Thurn.

Allen Tucker. Arts, 13:74-88, Feb. 1928; Int. Studio, 52: sup. 19-20, Mar. 1914.

Helen M. Turner. Book: Lee. Magazine: Art World, 3:490, Mar. 1918.

Walter Ufer. Amer. Mag. of Art, 13:507-14, Dec. 1922; Art and Archaeol. 16:66-74, Aug. 1923; *Fine Arts J. 34:221-6, May 1916; Int. Studio, 77:293-9, July 1923; Lit. Dig. 97:25, May 19, 1928.

R. W. Van Boskerck. Book: Isham.

†Theodore Van Soelen.

tDorothy Varian.

Abraham Walkowitz. Book: La Follette. Magazine: Int. Studio, 60: sup. 132-3, Feb. 1917.

†Nan Watson.

Frederick J. Waugh. Amer. Mag. of Art, 13: 15-18, Jan. 1922; Arts and Dec. 1:111-13, Jan. 1911; Century, 100:641-8, Sept. 1920; Gard. and Home B. 46:138-9, Oct. 1927; Int. Studio, 51: 273-8, Feb. 1914; Int. Studio, 60: sup. 50-2, Dec. 1916; Int. Studio, 74: sup. 125-35, Dec. 1921.

Max Weber. Book: *Cahill, Holger-Max Weber. Magazines: Amer. Mag. of Art, 21:419, July 1930; Arts, 16:514, Mar. 1930; *Menorah 
Jour. 11:580-2, Dec. 1925; Touchstone, 8:319-22, Jan. 1921.

$\dagger$ Harold Weston.

Arnold Wiltz. Art News, 28:15, Mar. 22, 1930.

Charles H. Woodbury. Art and Prog. 4:761-6, Nov. 1912; *Brush and Pencil, 6:1-12, Apr. 1900; Int. Studio, 42: sup. 71-8; Feb. 1911.

† Robert Strong Woodward.

Carl Wuermer. Art News, 28:12, Mar. 1, 1930; Lit. Dig., 106-17, Sept. 13, 1930.

N. C. Wyeth. Scribner's 66:638-42, Nov. 1919; Scribner's, 84:9-12, July, 1929; Scribner's, 84: front. Aug. 1928.

Marguerite Zorach. Art Digest, 4:9, Apr. 1, 1930; Art News, 28:12, Mar. 1, 1930; Arts, 16:494, Mar. 1930; Arts and Dec. 18:30, Jan. 1923; Int. Studio, 95:29-33, Mar. 1930. 
THE PUBLIC LIBRARY OF THE DISTRICT OF COLUMBIA REFERENCE LIST NO. 24

\section{CONTEMPORARY AMERICAN PAINTERS}

INCLUDED IN THE TWELFTH BIENNIAL EXHIBITION OF CONTEMPORARY AMERICAN OIL PAINTINGS

\section{THE CORCORAN GALLERY OF ART} NOVEMEER 30, 1930 - JANUARY 11, 1931 INCLUSIVE

ISSUED WITH THE COOPERATION OF THE CORCORAN GALLERY OF ART

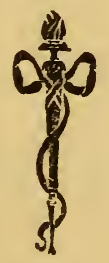

UNITED STATES

GOVERNMENT PRINTING OFFICE

WASHINGTON : 1930 


\section{CONTEMPORARY AMERICAN PAINTERS}

Brief biographical information in regard to most of the painters exhibiting is given in Who's Who in Art, 1929; Dictionary of American Painters, Sculptors, and Engravers, by Mantle Fielding; American Arts, by R. E. Jackson; The American Spirit in Art, by F. J. Mather, jr., and others; and Biographical Sketches of American Artists, published by the Michigan State Library. Critical comments on many of the exhibitors are in A Collection in the Making by Duncan Phillips. When an artist is represented in these reference works or by slight mention in any of the other books included in the list, no specific note is made.

A valuable clipping file of material on American artists is maintained by the Corcoran Gallery of Art. Nearly all of the artists exhibiting are represented in this collection, which may be consulted by persons interested. A dagger ( $t)$ in front of a name indicates that material about the artist has been found only in this file.

Under each exhibitor (so far as he has been written about) references are given to material in the books, referred to only by their authors, given at the beginning of the list. In cases where a number of articles have been written about an artist a selection has been made of the ones which seem to be the best.

The items marked with a star (*) are not in the Public Library but may nearly all be found at the Library of Congress. All other books and periodicals referred to in this list may be found in the Public Library, Eighth and K Streets NW.

The Public Library has published several reference lists on various phases of the fine arts which are still available. The assistants in the art division are always glad to help students of art history or criticism to locate needed material. 


\section{BOOKS}

Fielding, Mantle. Dictionary of American painters, sculptors, and engravers. 1926. W835F46. Reference.

Isham, Samuel. History of American painting, revised edition with supplementary chapters by Royal Cortissoz. 1927. WP83-Is3h.

Jackman, R. E. American arts. 1928. W83-J13.

La Follette, Suzanne. Art in America. 1929. W83-L13.

Lee, Cuthbert. Contemporary American portrait painters. 1929. WP83-L51.

Mather, F. J., jr., and others. The American spirit in art. (In the Pageant of America series.) 1927. F83-P144.

Michigan State Library. Biographical sketches of American artists. 5th ed. rev. 1924. W109 M584.

Phillips, Duncan. A collection in the making. 1926. WC859-P54.

Who's Who in Art. (In American art annual, 1929.) W-7Am. Reference.

\section{ARTISTS IN EXHIBITION}

Wayman Adams. Book: Lee. Magazines: Amer. Mag. 106: 24-7, Oct. 1928; Amer. Mag. of Art, 21: 640-8, Nov. 1930; Int. Studio, 77: 86-91, May, 1923; Lit. Dig. 100: 63, Jan. 5, 1929; Survey, 64: 318-19, July 1, 1930.

George G. Adomeit. *Art Dig. 4:15, Mar., 1930.

Ivan LeLorraine Albright. *Art. Dig. 5: 5, Oct. 1, 1930.

Karl Anderson. Book: Lee. Magazine: Int. Studio, 76: 132-4, Nov. 1922. 


\section{†James E. Bailey.}

†Burtis Baker.

†ozef G. Bakos.

†Kenneth Bates.

t Gustave Baumann.

Gifford Beal. Int. Studio, 44: sup. 20-30, Aug. 1911; Int. Studio, 77: 236-42, June, 1923; *Touchstone, 5: 240-3, June, 1919.

Oscar E. Berninghaus. Country Life, 33: 57-9, Apr. 1918.

Theresa Bernsiein. Book: Bryant LorindaAmerican pictures and their painters (WP83-B844). Magazine: Int. Studio 66: sup. 97-102, Feb. 1919. George Biddle. Amer. Mag. of Art, 20: 431-41, Aug. 1929.

Charles Bittinger. Book: Bryant LorindaAmerican pictures and their painters (WP83-B844). Magazine: Int. Studio, 59: sup. 41-4, Aug. 1916. Arnold Blanche. Art and Archaeol. 29: 39, Jan. 1930; Arts, 16: 419-21, Feb. 1930.

Ernest L. Blumenschein. Outlook 134: 504-7, Aug. 1, 1923.

Adolphe Borie. *Touchstone 4: 519-21, Mar. 1919.

†Frederick A. Bosley.

†Robert Brackman.

†Emile Branchard.

$\dagger$ Ross Braught.

Hugh H. Breckenridge. Amer. Mag. of Art, 7: 85-6, Jan. 1916. Int. Studio, 37: sup. 34-6, Mar. 1909.

†R. Sloan Bredin.

Alexander Brook. Art and Archaeol. 29: 34, 36, Jan. 1930; *Creative Art, 5: 713-18, Oct. 1929.

Roy Brown. Int. Studio, 83: 78-82, Jan., 1926. Margaret Fitzhugh Browne. Book: Lee. Amer. Mag. of Art, 16: 408-11, Aug. 1925, *Art News, 28:13, Dec. 7, 1929. 


\section{3}

Edward Bruce. Amer. Mag. of Art, 20: 480-1, Aug. 1929; Amer. Mag. of Art, 21: 75-81, Feb. 1930; Arts, 7: 273-5, May, 1925; Int. Studio, 88: 57-62, Dec. 1927; Int. Studio, 94: 82, Nov. 1929.

†Buk.

Charles Burchfield. Arts, 14: 5-12, July, 1928; Int. Studio, 80: 466-70, Mar. 1925; Int. Studio, 95: 27, Jan. 1930; Survey, 61: 159-61, Nov. 1, 1928. Bryson Burroughs. Book: Isham. Magazine: Arts and Dec. 12: 82-4, Dec. 28, 1919.

Emil Carlsen. Book: Isham. Magazines: Int. Studio, 61: sup. 105-10, June, 1917; Int. Studio, 75: 300-8, July, 1922; Int. Studio, 88: 53-60, Oct. 1927, Lad. H. J. 44: 10, Dec. 1927; Scribner's 66: 767-70, Dec. 1919; Touchstone, 7: 110-16, May, 1920.

John F. Carlson. Book: Bryant Lorinda-American pictures and their painters (WP83-B844). Magazines: *Apollo 11; 376, May, 1930, Review of his book, Elementary principles of landscape painting (WPS-C19); Art News, 28: 12, Feb. 22, 1930; *Fine Arts J. 35: 203-6, Mar. 1917.

John Carroll. Art News, 28: 22, May 24, 1930; *Colour, 13: 33, Sept. 1920; Int. Studio, 83: 75-9, Feb. 1926.

Clarence Holbrook Carter. *Cleveland Mus. Bul. 17: 86, 96, May, 1930; *Rochester Bul. 2:10, Nov. 1929.

Francis Chapin. *Art Dig. 4: 6, Apr. 1, 1930.

James Chapin. Int. Studio, 83: 75-9, Apr. 1926; Survey, 60: 464-7, Aug. 1, 1928.

C. K. Chatterton. Amer. Mag. of Art. 16: 308-10, June, 1925; Art and Archaeol. 5: 289-93, May, 1917.

Glenn O. Coleman. Book: La Follette. Magazine: Arts, 14: 261-5, Nov. 1928.

John E. Costigan. Int. Studio 80: 426-32, Mar. 1925.

†Catharine C. Critcher. 
Charles C. Curran. Book: Lee. Magazines: Critic 48: 38-9, Jan. 1906; Lit. Digest, 102: 23, Sept. 7, 1929.

John Steuart Curry. Arts, 16: 425, Feb. 1930; Survey, 64: 311-13, July 1, 1930.

Andrew Dasburg. *Arts, 6: 18-26, July, 1924.

Randall Davey. Amer. Mag. of Art, 7: 314-17, June, 1916; Int. Studio 75: 57-60, Mar. 1922.

Charles H. Davis. Book: Isham. Magazines: Int. Studio, 75: 176-83, June, 1922; New Eng. Mag. n. s. 27: 423-37, Dec. 1902.

$\dagger$ Julius Delbos.

F. Usher DeVoll. Amer. Mag. of Art, 21:150, Mar. 1930.

Ed win W. Dickinson. Lit. Digest, 103: 21, Nov. 30, 1929.

Sidney E. Dickinson. Amer. Mag. of Art, 21: 394, July, 1930.

Paul Dougherty. *Art Review 23: 1-3, Nov. 1911; Arts and Dec. 8: 251-4, Apr. 1918; Int. Studio, 30: 180-2, Dec. 1906; Int. Studio, 36: sup. 3-4, Nov. 1908; Int. Studio, 73: sup. 54-8, Apr. 1921.

Guy Pene Du Bois. Book: La Follette. Magazines: *Art in Amer. 18: 25-8, Dec. 1929; Arts and Dec. 4: 13-16, Nov. 1913.

W. Herbert Dunton. Amer. Mag. of Art, 15: 501-8, Oct. 1924.

†Charles Warren Eaton.

† William J. Edmondson.

† Walter Farndon.

Ernest Fiene. Arts, 16: 425, Feb. 1930.

† Gertrude Fiske.

John F. Folinsbee. Int. Studio, 73: sup. 38, Apr. 1921; Int. Studio, 76: 420-6, Feb. 1923.

Will Howe Foote. *Arts, Aug.-Sept., 1921.

Loren Ford. Survey, 64: 227-9, June 1, 1930. 
Frederick C. Frieseke. Art and Prog. 3: 747-9, Oet. 1912; Arts and Dec. 3: 13-15, Nov. 1912; Int. Studio, 53: 259-64, Oct. 1914; *Lad. H. J. 43: 16-17, July, 1926; Scribner's, 58: 643-6, Nov. 1915.

Maurice Fromkes. Art and Archaeol. 19: 162-74, Apr. 1925; *Art News, 28: 13, Dec. 7, 1929; Arts and Dec. 22: 34-5, Dec. 1924; Century, 77: 958, Apr. 1909; Country Life, 47: 86-90, Nov. 1924; Int. Studio, 80: 396-403, Feb. 1925; *Touchstone, 3: 302-8, July, 1918.

Daniel Garber. *Lad. H. J. 43: 20-1, May, 1926; * Maitres Contemporains 59: 10, 1912.

†S. Wood Gaylor.

Lillian Genth. Book: Bryant Lorinda-American pictures and their painters (WP83-B844). Magazines: Amer. Mag. of Art, 18: 227-37, May, 1927; *Fine Arts J. 37: 37-9, June, 1919; Int. Studio, 50: sup. 55-61, Oct. 1913; Lit. Dig. 100: 23, Mar. 16, 1929.

William Glackens. Books: Isham, La Follette. Magazines: Amer. Mag. of Art, 7:261-3, May, 1916; Arts, 3: 246-60, Apr. 1923; Arts and Dec. 4: 404-6, Sept. 1914; Arts and Dec. 7: 85, Dec. 1916; Arts and Dec. 14: 103, Dec. 1920; *Detroit Inst. Bul. 12: 7-8, Oct. 1930; Int. Studio, 40: sup. 68, May, 1910; Touchstone, 7: 191-9, June, 1920.

† Sudduth Goff.

Anne Goldthwaite. Int. Studio, 59: sup. 3-8, July, 1916.

Harry Gottlieb. Arts, 15: 99-102, Feb. 1929.

†John R. Grabach.

$\dagger$ J. Jeffrey Grant.

†W. Granville-Smith.

†Edwin Booth Grossman.

†Johanna K. W. Hailman.

Lilian Westcott Hale. Amer. Mag. of Art, 18: 58-70, Feb. 1927. 
Philip L. Hale. Amer. Mag. of Art. 7: 259-60, May, 1916; *World Today, 14: 59-67, Jan. 1908.

George Harding. Amer. Mag. of Art 10: 451-6, Oct. 1919. Int. Studio 62: sup. 16-18, July, 1917.

Bertram Hartman. Book: La Follette.

Childe Hassam. Books: La Follette, McGuire, J. C.-Childe Hassam (In American Etchers, v. 3, W10-H277a); McSpadden, J. W.-Famous painters of America (WP83-M246); Poussette-Dart, Nathaniel, comp.-Childe Hassam (W10-H277, Ref.). Magazines: Art and Prog. 2: 137-9, Mar. 1911; Int. Studio, 45: sup. 29-30, Dec. 1911; Int. Studio, 57: sup. 83-6, Jan. 1916; Int. Studio, 77: 2-7, Apr. 1923; Int. Studio, 93: 78, June, 1924; Nation, 101: 698-9, Dec. 9, 1915; Scribner's 84: 415-22, Oct, 1928; Touchstone, 5: 272-80, July, 1919.

Charles W. Hawthorne. Books: Isham, Lee. Magazines: Art and Archaeol. 28: 87-8, Sept. 1929; Art and Prog. 4: 821-5, Jan. 1913; Int. Studio, 26: 261-4, Sept. 1905; Int. Studio, 37: sup. 65-8, May 1909; Int. Studio, 61: sup. 19-24, Mar. 1917; Lit. Dig. 91: 27; Nov. 6, 1926; Lit. Dig. 98: 30, Aug. 25, 1928.

$\uparrow$ Norbert Heermann.

tMartin Hennings.

Henry Hensche. *Art Dig. 4: 6, Apr. 1, 1930.

Harry Hering. Arts, 16: 342, Jan. 1930.

Aldro T. Hibbard. Art News, 28: 15-16, Jan. 25, 1930.

Eugene Higgins. Arts and Dec. 5: 134-5, 164-5, Feb. 1915; Craftsman, 12: 134-46, May 1907; Cur. Lit. 43: 401-4, Oct. 1907; *Fine Arts J. Oct. 1907; Survey, 59: 562-3, Feb. 1, 1928; *Touchstone, 5: 360-9, Aug. 1919.

$\dagger$ Victor Higgins.

tClarence K. Hinkle.

Stefan Hirsch. *Newark Museum 2: 54, Apr. 1929. 
Charles Hopkinson. Book: Lee. Magazine: Nation 92: 369-70, Apr. 13, 1911.

Edward Hopper. Book: La Follette. Magazines: *Arts, 5: 323, June 1924; Arts, 11: 134-8, Mar. 1927; *Shadowland, Oct. 1922, pp. 22-3.

Ernest L. Ipsen. Book: Lee. Amer. Mag. of Art, 21: 195, Apr. 1930.

John C. Johansen. Book: Lee. Magazines: Amer. Mag. of Art 10: 290-5, June, 1919; Int. Studio, 26: 264-7, Sept. 1905; Int. Studio, 42: sup. 3-6, Nov. 1910.

$\dagger$ J. Theodore Johnson.

Morris Kantor. Book: Phillips.

Bernard Karfiol. *Arts, 6: 76-82, Aug. 1924.

Sergeant Kendall. Book: Isham. Harper's, 117: 568-77, Sept. 1908.

Rockwell Kent. Books: Isham, Kent-Voyaging southward from the Strait of Magellan (G995T-K4), Wilderness (G945-K418). Magazines: Amer. Mag. 99: 18-21, Mar. 1925; *Architecture (N. Y.), 61: 285-8, May 1930; Arts and Dec. 11: 70-2, June, 1919; Arts and Dec. 12: 324-5, Mar. 1920; Country Life, 56: 35-8, July, 1929; Cur. Opinion, 62: 277-8, Apr. 1917; Cur. Opinion, 67: 52-3, July 1919; Cur. Opinion, 68: 681-5, May 1920; Int. Studio, 67: sup. 105-12, June, 1919; Int. Studio, 79: 272-6, July, 1924; Lit. Dig. 61: 31-2, May 31, 1919; Lit. Dig. 106: 17-18, Aug. 23, 1930; New Repub. 50: 302-3, May 4, 1927; Outlook, 155: 391-3, July 9, 1930; Wilson Bul. 4: 356, Apr. 1930.

Georgiana Klitgaard. Arts, 16: 118, Oct. 1929.

Karl Knaths. Art and Understanding, 1: 110-14, Nov. 1929; Art. News, 28: 12, Mar. 1, 1930.

Leon Kroll. *Art in Amer. 18: 299-303, Oct. 1930; *Canadian Bookman, 12: 39, Feb. 1930; Int. Studio, 72: 36-8, Feb. 1921. 
Louis Kronberg. Amer. Mag. of Art, 19: 194-200, Apr. 1928; Int. Studio, 28: 74-5, Mar. 1906; New Eng. Mag. n. s. 38: 498-9, June 1908.

Yasuo Kuniyoshi. Arts, 5: 22-7, Jan. 1924; Arts and Dec. 7: 251-3, Mar. 1917; Dial, 76: 295-7, Mar. 1924, *Trans-Pacific, 13: 10, Oct. 23, 1926.

†Richard Lahey.

William L. Lathrop. Amer. Mag. 104: 34-5, Nov. 1927; Int. Studio, 78: 132-8, Nov. 1923.

Ernest Lawson. Amer. Mag. of Art 8: 257-63, May 1917: *Art in Amer. 8: 32-39, Dec. 1919; Arts and Dec. 6: 505-7, Sept. 1916; Arts and Dec. 10: 257-8, Mar. 1919; *Canadian Bookman, 12: 39, Feb. 1930; Cur. Lit. 42: 406-9, Apr. 1907; Int. Studio, 59: sup. 13-15, July 1916; Int. Studio, 72: sup. 114-17, Feb. 1921; Int. Studio, 78: 366-70, Feb. 1924.

Jonas Lie. Book: Bryant Lorinda-American pictures and their painters (WP83-B844). Magazines: Amer. Mag. of Art, 16: 58-66, Feb. 1925; Art News, 29: 6, Oct. 11, 1930; Arts and Dec. 12: 91, Dec. 1919; Arts and Dec. 15: 221, Aug. 1921; Country Life, 36: 55-7, July 1919; Craftsman, 13: 135-9, Nov. 1907; Cur. Lit. 52: 222-4, Feb. 1912; Int. Studio, 51: sup. 192-4, Feb. 1914; Int. Studio 82: 102-7, Nov. 1925; Pan Amer. Union Bul. 38: 679-83, May 1914.

Luigi Lucioni. Art News, 28:10, Feb. 8, 1930.

George Luks. Books: La Follette, Lee. Magazines: Outlook, 154: 34, Jan. 1, 1930; Rev. of Revs. 80: 82-3, Sept. 1929; Survey, 55: 20-4, Oct. 1, 1925; Touchstone, 8: 32-8, Oct. 1920.

Stewart S. MacDermott. Art News, 28: 12, Jan. 4, 1930; *Creat. Art, 6: sup. 14, Jan., 1930; Int. Studio, 95: 76, Feb. 1930.

†Henry Lee McFee. 
M. Jean McLane. Book: Lee. Magazines: Art and Prog. 3: 612, June, 1912; Arts and Dec. 3: 299-301, July, 1913; Harper's 118: 291-3, Jan., 1909.

Emma Fordyce MacRae. *Art Digest, 4: 17, Mar. 15, 1930; Art News, 28: 10, Feb. 15, 1930.

Kristjan Magnusson. Amer. Mag. of Art, 21: 435-8, Aug. 1930.

$\dagger$ Peppino Mangravite.

†Alexis B. Many.

Henry Mattson. Arts 15: 26-9, Jan., 1929.

Gari Melchers. Books: Brinton, ChristianModern artists (W10-9B774), Isham, Lee. Magazines: Amer. Mag. of Art, 13: 117-21, Apr., 1922; Amer. Mag. of Art, 15: 79-85, Feb., 1924; Arts and Dec. 24: 40-1, Nov., 1925; Harper's, 114: 430-9, Feb., 1907; Int. Studio, 31: sup. 11-18, Mar., 1907; Int. Studio, 48; sup. 27-30, Dec. 1912; *Lad. H. J. 43: 26-7, Dec. 1926; Mentor, 17: 36-7, Aug., 1929; World's Work 15: 10092-105, Apr. 1908.

$\dagger$ Richard S. Meryman.

William Meyerowitz. *Art Digest, 4: 22, Mar. 1, 1930.

Kenneth Hayes Miller. Books; *Goodrich, L.Kenneth Hayes Miller (pamphlet); La Follette. Magazines: *Art in Amer. 18: 86-92, Feb., 1930; *Art News, 28: 13, Dec. 7, 1929; Arts, 14: 300-7, Dec. 1928; Arts, 16: 641, May, 1930; New Repub. 27: 244-5, July 27, 1921 .

Richard E. Miller. Art and Prog. 6: 39-40, 54-7, Dec., 1914; Int. Studio, 43: 263-73, June, 1911.

†Ross Moffett.

$\nmid$ A. Molarsky.

$\nmid$ Maurice Molarsky.

H. Dudley Murphy. *Sketch Book, 6: 303-6, Nov., 1907. 
Jerome Myers. Books: Caffin, C. H.-Story of American painting (WP83-C11), Isham, La Follette. Magazines: Amer. Mag. of Art, 8:481-5, Oct. 1917; Art and Prog. 5:89-94, Jan. 1914; Arts and Dec. 10:257, 259, Mar. 1919; Craftsman, 29:25-32, Oct. 1915; Int. Studio, 57 : sup. 125-8, Feb. 1916; Survey, 51:33-9, Oct. 1, 1923; *Touchstone, 5:396-9, Aug. 1919.

Warren Newcombe. *Art Dig. 4: 18, Mar. 15, 1930.

John Noble. Amer. Mag. 104:34-5, Aug. 1927; Int. Studio, 77:457-65, Sept. 1923; Outlook, 155:153, May 28, 1930. 1926.

B. J. O. Nordfeldt. Survey, 57:132-3, Nov. 1,

Nura. Art News, 28:13, Apr. 19, 1930.

George Oberteuffer. Int. Studio, 43:275-80, June, 1911.

Ivan G. Olinsky. Art News, 29:11, Oct. 11, 1930; Lit. Dig. 83:29, Nov. 15, 1924.

$\dagger J$. Olaf Olson.

William M. Paxton. Book: Lee. Magazines: Int. Studio, 39: sup. 46-48, Dec. 1909; Lit. Dig. 89:25, June 19, 1926; *World Today, 9:1210-14, Nov. 1905.

†Joseph T. Pearson.

Charles H. Pepper. *World Today, 9:1271-8, Dec. 1905.

Van Deering Perrine. Arts and Dec. 21:14-15, May, 1924; Century, 72:165-71, Sept. 1906; Craftsman, 12:486-96, Aug. 1907; Cur. Lit. 41:40710, Oct. 1906; *Touchstone, 6:8-9, Oct. 1919.

Marjorie Phillips. Book: La Follette. Magazine: Art News, 28:20, Mar. 22, 1930.

Salvatore Pinto. Art News, 28:10, Jan. 11, 1930.

Ogden M. Pleissner. Art News, 28:16, Jan. 11, 1930.

Joseph Pollet. *Creat. Art, 6:32-9, Jan. 1930 
Abram Poole. Art and Archaeol. 29:129, Mar. 1930; Arts and Dec. 23:54, Sept. 1925; *Colour, n. s. $1: 10$, Jan. 1925 .

Henry Rankin Poore. Book: Isham. Magazines: Amer. Mag. of Art, 21:543, Sept. 1930, Review of his book, *"Art principles in practice for the student and layman in art"; Country Life 42:34-9, Sept. 1922; Dial, 35:174, Sept. 16, 1903, Review of his book, "Pictorial composition and the critical judgment of pictures" (W-P795); Int. Studio, 37: sup. 20-4, Mar. 1909; New Eng. Mag. n. s. 39:604-5, Jan. 1909.

M. Elizabeth Price. Art News, 28:10, Mar. 8, 1930.

$\dagger$ Norris Rahming.

Ellen Emmet Rand. Book: Lee. Magazine: Amer. Mag. of Art, 19:471-9, Sept. 1928.

Edward W. Redfield. Book: Isham. Magazines: Amer. Mag. of Art, 16:3-8, Jan. 1925; Amer. Mag. of Art, 21:139-42, Mar. 1930; Arena, 36: 20-6, July, 1906; Arts and Dec. 6:135, Jan. 1916; Country Life, 13:194-7, Dec. 1907; Country Life, 38:38-9, June, 1920; Int. Studio, 41: sup. 29-36, Aug. 1910; Int. Studio, 75:402-10, Aug. 1922; *Lad. H. J. 43:20-1, May, 1926; Lit. Dig. 92:29, Jan. 22, 1927.

†Umberto Romano.

tCharles Rosen.

† Margery Austen Ryerson.

† Margarett Sargent.

Charles Schlein. Art News, 28:15, May 10, 1930.

Henry Schnakenberg. *Art Digest, 4:6, Apr. 15, 1930; Int. Studio, 84:58-62, Aug. 1926.

W. Elmer Schofield. Book: Isham. Magazines: Arts and Dec. 1:473-5, Oct. 1911; Int. Studio, 48:280-6, Feb. 1913. 
Leopold Seyffert. Books: Bryant LorindaAmerican pictures and their painters (WP83-B844), Lee. Magazines: Amer. Mag. of Art, 20:64-73, Feb. 1929; Int. Studio, 84: 84, July, 1926.

\section{†John Sharman.}

Edward Simmons. Books: Bryant LorindaAmerican pictures and their painters (WP83-B844), Isham, Simmons Edward-From 7 to 70 (W10-Si46). Magazine: Cur. Opinion, 74:58-60, Jan. 1923.

Gerrit V. Sinclair. *Milwaukee Inst. Bul. 3: 5, Apr. 1930.

John Sloan. Books: Gallatin, A. E. ed.-John Sloan (W10-Sl52), La Follette. Magazines: Amer. Mag. of Art, 20:554-9, Oct. 1929, Amer. Mag. of Art, 21:282, May, 1930; Arts, 11:168-78, Apr. 1927; Craftsman, 15:559-64, Feb. 1909; Harper's Weekly, 58:20-1, Nov. 22, 1913; Int. Studio, 58: sup. 25-8, Mar. 1916; Lit. Dig. 97:26-7, Apr. 21, 1928; Theatre Arts Mag. 14:679-82, Aug. 1930; *Touchstone, 4:362-70, Feb. 1919; Va. Q. R. 1:196-204, July, 1925.

Eugene Speicher. Book: Bryant LorindaAmerican pictures and their painters (WP83-B844). Magazines:*Arts, 6: 314-27, Dec. 1924; Int. Studio, 46: sup. 19-22, Mar. 1912; Int. Studio, 95:26, Jan. 1930; New Repub. 58:280, Apr. 24, 1929.

$\dagger$ Francis Speight.

Robert Spencer. Int. Studio, 76:485-91, Mar. 1923.

Maurice Sterne. Book: Birnbaum, Martin-Introductions (W10-9B5361). Magazines: Art and Understanding, 1:86-9, Nov. 1929: Art News, 28:22, Feb. 1, 1930; Cur. Opinion, 59:425-7, Dec. 1915; Int. Studio, 46: sup. 3-13, Mar. 1912; Int. Studio 61: sup. 53-6, Apr. 1917; Int. Studio, 95:27, 35, 58, Jan. 1930; New Repub. 33:149, Jan. 3, 1923; New Repub. 45:355, Feb. 17, 1926; New Repub. 61:142-3, Dec. 25, 1929 
Albert Sterner. Books: Isham, Lee. Magazines: Art News 28:13, Apr. 5, 1930; Arts and Dec. 24:42-4, Feb. 1926; Bookman 10:550-4, Feb. 1900; Int. Studio, 13:78-80, Mar. 1901; Int. Studio, 52: sup. 71-8, May, 1914; Outlook 137:99-102, May 21, 1924; Print Col. Q. 6:213-24, Apr. 1916; Putnam's 2:387-99, July 1907.

Alice Kent Stoddard. Amer. Mag. of Art, 21: 480, Sept. 1930.

Anthony Thieme. Amer. Mag. of Art, 21: 531, Sept. 1930; Lit. Dig. 103: 28, Oct. 5, 1929; *Wadsworth Ath. Bul. 8: 24, Apr. 1930.

$\dagger$ Leslie $P$. Thompson.

tErnest Thurn.

Allen Tucker. Arts, 13:74-88, Feb. 1928; Int. Studio, 52: sup. 19-20, Mar. 1914.

Helen M. Turner. Book: Lee. Magazine: Art World, 3:490, Mar. 1918.

Walter Ufer. Amer. Mag. of Art, 13:507-14, Dec. 1922; Art and Archaeol. 16:66-74, Aug. 1923; *Fine Arts J. 34:221-6, May 1916; Int. Studio, $77: 293-9$, July 1923; Lit. Dig. 97:25, May 19, 1928.

R. W. Van Boskerck. Book: Isham.

†Theodore Van Soelen.

tDorothy Varian.

Abraham Walkowitz. Book: La Follette. Magazine: Int. Studio, 60: sup. 132-3, Feb. 1917.

$\dagger$ Nan Watson.

Frederick J. Waugh. Amer. Mag. of Art, 13: 15-18, Jan. 1922; Arts and Dec. 1:111-13, Jan. 1911; Century, 100:641-8, Sept. 1920; Gard. and Home B. 46:138-9, Oct. 1927; Int. Studio, 51: 273-8, Feb. 1914; Int. Studio, 60: sup. 50-2, Dec. 1916; Int. Studio, 74: sup. 125-35, Dec. 1921.

Max Weber. Book: *Cahill, Holger-Max Weber. Magazines: Amer. Mag. of Art, 21:419, July 1930; Arts, 16:514, Mar. 1930; *Menorah 
Jour. 11:580-2, Dec. 1925; Touchstone, 8:319-22, Jan. 1921.

†Harold Weston.

Arnold Wiltz. Art News, 28:15, Mar. 22, 1930.

Charles H. Woodbury. Art and Prog. 4:761-6, Nov. 1912; *Brush and Pencil, 6:1-12, Apr. 1900; Int. Studio, 42: sup. 71-8, Feb. 1911.

†Robert Strong Woodward.

Carl Wuermer. Art News, 28:12, Mar. 1, 1930; Lit. Dig., 106-17, Sept. 13, 1930.

N. C. Wyeth. Scribner's 66: 638-42, Nov. 1919; Scribner's, 84: 9-12, July, 1929; Scribner's, 84: front. Aug. 1928.

Marguerite Zorach. Art Digest, 4:9, Apr. 1, 1930; Art News, 28:12, Mar. 1, 1930; Arts, 16:494, Mar. 1930; Arts and Dec. 18:30, Jan. 1923; Int. Studio, 95:29-33, Mar. 1930. 
